\title{
The flora of vascular plants in Gibaeksan Mt. County Park and mountains neighboring the park
}

\author{
Beom Kyun PARK ${ }^{1,2}$, Dong Chan $\mathrm{SON}^{2}$ and Sung Chul KO ${ }^{1 *}$ \\ ${ }^{1}$ Department of Biological Science and Biotechnology, Hannam University, Daejeon 34054, Korea \\ ${ }^{2}$ Division of Forest Biodiversity, Korea National Arboretum, Pocheon 11186, Korea \\ (Received 14 February 2020; Revised 29 May 2020; Accepted 18 June 2020)
}

\begin{abstract}
The flora of vascular plants in the Gibaeksan Mt. County Park and its neighboring mountains, located at the boundary between Geochang-gun and Hamyang-gun in Gyeongsangnam-do province in Korea, were surveyed for a total 46 times from April to September of 2011, in July of 2012, and from April of 2015 to August of 2018. The result of this survey revealed 659 taxa composed of 107 families, 346 genera, 583 species, 14 subspecies, 46 varieties and 6 forms. Among them, 25 taxa were endemic plants to Korea, and 18 taxa were rare and endangered plants of Korea. The floristic regional indicator plants including cultivated plants were 5 taxa of grade V, 5 taxa of grade IV, 29 taxa of grade III, 30 taxa of grade II and 38 taxa of grade I. Forty-three taxa of alien plants were found in this area. In addition, 500 taxa out of a total of 649 taxa were categorized by usage into eight groups, including among others an edible group containing 257 taxa, a medicinal group containing 206, a pasturing group containing 220, and an ornamental group containing 84 , with some taxa belonging to more than one group. The flora of this surveyed area belongs to the southern province among the floral provinces of the Korean Peninsula.
\end{abstract}

Keywords: Flora, Gibaeksan Mt. county park, Geumwonsan Mt., Gibaeksan Mt., Hwangseoksan Mt., Geomangsan Mt.

기백산군립공원은 지리적으로 소백산맥에 속하는 남 덕유산 $(1,507.4 \mathrm{~m})$ 에서 남동쪽으로 뻗어나간 지맥의 $8.5 \mathrm{~km}$ 지점에 위치한다. 본 조사 범위에는 기백산 군립 공원과 인근지역의 산지로서 금원산 $(1,352.5 \mathrm{~m})$, 기백산 $(1,350.8 \mathrm{~m})$, 황석산 $(1,190 \mathrm{~m})$ 과 거망산 $(1,184 \mathrm{~m})$ 이 포함되 었다. 행정구역상으로 금원산 $(1,352.5 \mathrm{~m})$ 은 경상남도 거 창군 북상면과 위천면 및 함양군 안의면의 경계에, 기백 산 $(1,350.8 \mathrm{~m})$ 은 위천면과 안의면의 경계에, 황석산 $(1,190 \mathrm{~m})$ 과 거망산 $(1,184 \mathrm{~m})$ 은 경상남도 함양군 안의면, 서상면과 서하면의 경계에 각각 위치하고 있다. 전체적 으로 본 조사지는 동쪽으로는 금원산, 기백산으로, 서쪽 으로는 은신치, 거망산, 황석산으로 이어지는 능선이 각 각 있으며, 이들 능선은 금원산과 거망산의 사이에 있는 수망령에 의해 이어져 있다.

본 산지의 수계는 서쪽으로는 남강, 북동쪽으로는 월 성계곡, 북쪽으로는 창선천, 동쪽으로는 한수동계곡과 유안청계곡, 거망산과 금원산 사이의 수망령에서 시작하 는 용추계곡이 흐르고 있다. 용추계곡은 함양군 안의면에 서 지우천으로 유입되어 남강으로 합류되며, 월성계곡, 창
선천, 유안청계곡과 한수동계곡은 위천천으로 유입되어 황강의 상류를 이루고, 합천호로 흘러든다.

토양은 여름과 겨울에 기온과 강우량의 교차가 큰 낙엽 활엽수림대에서 형성되는 갈색산림토로서 표층이 두텁 고 용탈이 적어서, 전반적으로 표층은 갈색을 띄고, 하위 층은 황갈색산림토이다. 암석은 주로 화강편마암과 화강 암으로 구성되어 있으며, 화강편마암은 차별침식으로 산 릉을 이루고 있다(Kang, 1971).

본 조사지가 속한 거창군과 함양군의 기후는 연교차와 일교차가 크고, 여름철에는 덥고 강우의 $60-70 \%$ 가 편중 되며, 겨울철에는 추운 전형적인 내륙성 기후를 보이기는 하나, 해발 $1,000 \mathrm{~m}$ 이상의 높은 산들이 곳곳에 위치하고 있어 겨울철의 북서풍과 여름철의 계절풍인 남동풍을 막 아주어 바람으로 인한 영향은 없는 편이다. 한편 최근 5년 간 기상자료에 의하면 거창군의 연평균기온은 $12.3^{\circ} \mathrm{C}$, 연 평균 강수량은 $1,074.4 \mathrm{~mm}$ 이며, 함양군의 연평균기온은 $13.0^{\circ} \mathrm{C}$, 연평균 강수량은 $1,087.5 \mathrm{~mm}$ 이다(Geochang-gun, 2018; Hamyang-gun, 2018). 최한월은 1월이며, 최난월은 8 월이고, 연교차는 거창군이 $26.7^{\circ} \mathrm{C}$, 함양군이 $26.4^{\circ} \mathrm{C}$ 이다.

\footnotetext{
*Author for correspondence: kscaaa@hnu.kr
} 
본 조사지역은 한반도 식물의 지리적 분포를 바탕으로 구분된 8개의 식물구계 중 남부아구에 해당되며(Lee and Yim, 1978, 2002), 한반도 기후를 바탕으로 한 5개 산림대 중 온대남부대에 속한다(Yim and Kira, 1975).

본 산지의 식물상에 관한 선행연구로는 금원산에 대하 여 Kwon et al. (1997)은 금원산 자연휴양림의 목본식물상 을 조사하여 49과 87속 147분류군을, Seok et al. (2003)은 108과 357속 534종 80변종 6품종 620분류군을, Park et al. (2007)은 금원산 자연휴양림 산림식생의 종조성을 조사 하면서 교목 27종 아교목 44종 관목 71종 초본 69종의 총 152분류군을, Oh et al. (2008)은 금원산 생태수목원 조성 부지의 관속식물상 연구에서 84과 217속 319종 43변종 7 품종 369분류군을 각각 보고하였다. 그리고 Lee and Oh (1986)가 함양 백운산 일대 종합보고에서 황석산과 기백 산을 포함하여 96과 297속 499분류군을, Lee (1986)는 78 과 194속 335분류군을, $\operatorname{Kim}$ (2011)은 기백산에서 108과 383 속 587 종 114 변종 21 품종 722 분류군을 각각 조사·보 고한 바 있으며, National Institute of Environmental Research (2012)와 Oh et al. (2015)에 의해 97과 295속 394 종 4아종 80 변종 17 품종 495 분류군이, Shin (2015)에 의해 61 과 132 속 167 종 2 아종 22 변종 4 품종 195 분류군이, 거망
산은 Jeong (2017)에 의해 76과 188속 246종 4아종 31변종 6 품종의 총 287 분류군이 각각 보고된 바 있다. 그러나 상 기 연구들의 소산식물목록 중 큰천남성, 히어리, 도깨비 부채, 자주종덩굴, 검종덩굴 등 본 조사지역에 분포가 의 심되는 식물들이 다수 포함되어 있고, 이들에 대한 증거 표본의 언급이 없기 때문에 현재 식물상과의 비교 연구 를 수행하는데 어려움이 있다.

본 연구는 기백산군립공원 일대의 금원산, 기백산, 거망 산, 황석산에 대한 현지조사를 통해 수집한 증거표본을 바탕으로 관속식물상을 조사하여 선행연구들과 그 결과 를 비교 검토하고, 본 지역의 식물지리학적 특징과 주요 식물을 파악하여, 기백산군립공원의 주요 산림식물자원 에 대한 분포와 이들의 보존 및 관리정책을 수립하기 위 한 기초자료를 제공하고자 수행되었다.

\section{재료 및 방법}

\section{조사경로 및 일정}

조사 범위와 경로는 기백산군립공원을 중심으로 북쪽 으로는 금원산 북사면, 동쪽으로는 기백산 동사면, 남쪽 으로는 황석산 남사면, 서쪽으로는 거망산 서사면 일대를

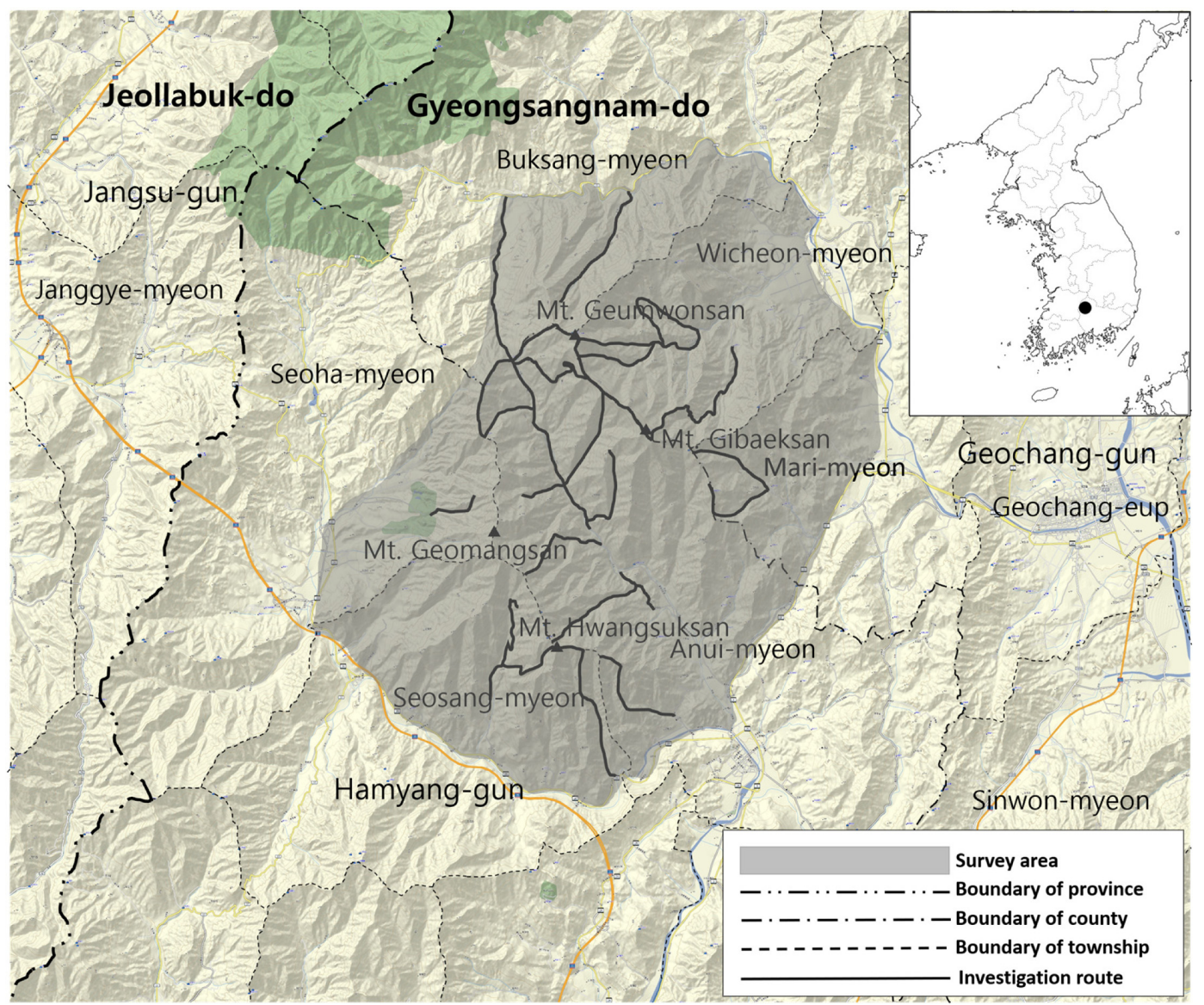

Fig. 1. Map of investigated area and routes in Geochang-gun and Hamyang-gun. 
경계로 하여 식물상이 비교적 다양하다고 생각되는 지역 을 선정하였고, 현지조사는 계곡, 등산로, 임도를 따라 사 면별, 고도별로 이루어졌다(Fig. 1).
조사 기간은 2011년 4월에서 9월까지, 2012년 7월, 2015 년 4월부터 2018년 8월까지 총 46회에 걸쳐 행해졌으며, 자세한 일정별 경로는 Table 1과 같다.

Table 1. Dates and routes of the investigation.

\begin{tabular}{|c|c|}
\hline Date & Route of investigation \\
\hline 20 Apr 2011 & Ticket office - Jawoonpokpo (falls) - Yuancheongpokpo (falls) - gazebo - summit of Geumwonsan Mt. \\
\hline 14 May 2011 & $\begin{array}{l}\text { Pogojang (village) - Sumangnyeong (uphill) - summit of Geumwonsan Mt. - heliport - forest road - Yuancheongpokpo (falls) - } \\
\text { Jawoonpokpo (falls) - ticket office }\end{array}$ \\
\hline 15 May 2011 & Ticket office - Jawoonpokpo (falls) - Yuancheongpokpo (falls) - forest road \\
\hline 18 May 2011 & A : ticket office - Munbawi (stone) - Jijaemigol (valley) - forest road \\
\hline & B : ticket office - Jawoonpokpo (falls) - Yuancheongpokpo (falls) - forest road \\
\hline 19 May 2011 & A : Jawoonpokpo (falls) - Yuancheongpokpo (falls) - forest road - heliport - summit of Geumwonsan Mt. \\
\hline & B : Changseon-ri (village) - Changseoncheon (river) \\
\hline 4 Jun 2011 & Ticket office - Munbawi (stone) - Jijaemigol (valley) - forest road - Yuancheongpokpo (falls) - Jawoonpokpo (falls) - ticket office \\
\hline 16 Jul 2011 & $\begin{array}{l}\text { Sapyeong (village) - Siheung-gol (valley) - milestone (three-way) - gazebo - summit of Geumwonsan Mt. - heliport - forest } \\
\text { road - Yuancheongpokpo (falls) - Jawoonpokpo (falls) - ticket office }\end{array}$ \\
\hline 30 Aug 2011 & $\begin{array}{l}\text { Ticket office - Munbawi (stone) - Jijaemi (valley) - forest road - summit of Geumwonsan Mt. - heliport - forest road - } \\
\text { yuancheongpokpo(falls) - Jawoonpokpo (falls) - ticket office }\end{array}$ \\
\hline 18 Sep 2011 & $\begin{array}{l}\text { Pogojang (village) - Sumangnyeong (uphill) - summit of Geumwonsan Mt. - heliport - forest road - Yuancheongpokpo (falls) - } \\
\text { Jawoonpokpo (falls) - ticket office }\end{array}$ \\
\hline $21 \mathrm{Jul} 2012$ & Sapyeong (village) - Siheung-gol (valley) - milestone (three-way) - Siheunggol (valley) - Sapyeong (village) \\
\hline 24 Apr 2015 & Yongchusa iljumun(gate) - starting point of Deulmeori - Dosugol (valley) - Dosuk (valley) \\
\hline 5 Jun 2015 & Yongchusa iljumun(gate) - Sapyeong - Yongchu natural recreation forest - Sumangnyeong (uphill) \\
\hline 9 Jul 2015 & Sangchonmaeul (village) - Neulbatgogae (hill) \\
\hline 12 Aug 2015 & Sangchonmaeul (village) - Jeolteosgol (valley) - Anbong (Mt.) \\
\hline 17 Sep 2015 & Sumangnyeong (uphill) - forest road - heliport - summit of Gibaeksan Mt. - heliport - forest road - Sumangnyeong (uphill) \\
\hline 18 Sep 2015 & Sapyeong (village) - Siheunggol (valley) - ridge of Gibaeksan Mt. \\
\hline 15 Oct 2015 & Sangcheonjeosuji (reservoir) - Hansudong valley - summit of Gibaeksan Mt. \\
\hline 16 Oct 2015 & Yongchu natural recreation forest - Sumangnyeong (uphill) - forest road - heliport \\
\hline 23 Apr 2015 & Deokhyeonmaeul (village) - Sannaegol (valley) - Pibawi(stone) - Geobukbawi (stone) \\
\hline 24 Apr 2015 & Yongchusa iljumun(gate) - starting point of Deulmeori - Dosugol (valley) - Dosuk (valley) \\
\hline 19 May 2015 & Ujeonmaeul (Village) - Sigumokgol (valley) \\
\hline 4 Jun 2015 & Hwangamsa (temple) - $710 \mathrm{~m}$ highland \\
\hline 5 Jun 2015 & Yongchusa iljumun(gate) - Sapyeong (village) - Yongchu natural recreation forest - Sumangnyeong (uphill) \\
\hline 9 Jul 2015 & Sangchonmaeul (village) - Neulbatgogae (hill) \\
\hline $10 \mathrm{Jul} 2015$ & $\begin{array}{l}\text { Ujeonmaeul (Village) - Pibawi (stone) - Hwangseoksanseong (Fortress) - Hwangseoksanseong nammun (fortress) - summit of } \\
\text { Hwangseoksan Mt. }\end{array}$ \\
\hline 12 Aug 2015 & Sangchonmaeul (village) - Jeolteosgol (valley) - Anbong (Mt.) \\
\hline 13 Aug 2015 & Deokhyeonmaeul (village) - Sannaegol (valley) - Deokhyeonmaeul (village) - Yudongmaeul (village) - Yeonchonmaeul (village) \\
\hline 17 Spe 2015 & Sumangnyeong (uphill) - forest road - heliport - summit of Gibaeksan Mt. - heliport - forest road - Sumangnyeong (uphill) \\
\hline 18 Sep 2015 & Yongchu natural recreation forest - Sapyeong (village) - Siheunggol (valley) - milestone (three-way) \\
\hline 15 Oct 2015 & Sangcheonjeosuji (reservoir) - Hansudonggyegok (valley) - forest road - milestone (three-way) - summit of Gibaeksan Mt. \\
\hline 16 Oct 2015 & Yongchu natural recreation forest - Sumangnyeong (uphill) - forest road - heliport \\
\hline
\end{tabular}


Table 1. Continued.

\begin{tabular}{|c|c|}
\hline Date & Route of investigation \\
\hline 22 Oct 2015 & $\begin{array}{l}\text { Ujeonmaeul (village) - Pibawi (stone) - Hwangseoksanseong (fortress) - Hwangseoksanseong nammun (fortress) - summit of } \\
\text { Hwangseoksan Mt. }\end{array}$ \\
\hline 23 Oct 2015 & Hwangamsa (temple) - summit of Hwangseoksan Mt. \\
\hline 15 Apr 2016 & $\begin{array}{l}\text { ticket office - Jawoonpokpo (falls) - Yuancheongpokpo (falls) - gazebo - summit of Geumwonsan Mt. - heliport - forest road - } \\
\text { Geumwonsan Mt. Recreational Forest - ticket office }\end{array}$ \\
\hline 21 Sep 2016 & $\begin{array}{l}\text { Ujeonmaeul (village) - Pibawi (stone) - Hwangseoksanseong (fortress) - Hwangseoksanseong nammun (fortress) - summit of } \\
\text { Hwangseoksan Mt. }\end{array}$ \\
\hline 22 Sep 2016 & Sapyeong (village) - Taejanggol (valley) \\
\hline 25 May 2017 & Cheongnyangsa (temple) - Buldanggol (valley) - milestone (three-way) \\
\hline \multirow[t]{2}{*}{26 May 2017} & A: Yongchusa iljumun(gate) - Yongchugyegok (valley) - Sapyeong (village) \\
\hline & B: Deokhyeonmaeul (village) - Sannaegol (valley) - Pibawi (stone) \\
\hline $13 \mathrm{Jul} 2017$ & Cheongnyangsa (temple) - Buldanggol (valley) - milestone (three-way) - milestone (three-way) \\
\hline $14 \mathrm{Jul} 2017$ & $\begin{array}{l}\text { Ujeonmaeul (Village) - Pibawi (stone) - Hwangseoksanseong (Fortress) - Hwangseoksanseong nammun (gate) - summit of } \\
\text { Hwangseoksan Mt. }\end{array}$ \\
\hline 18 Apr 2018 & Sapyeong (village) - Taejanggol (valley) - Sapyeong (village) - forest road - milestone (three-way) - Jijanggol (valley) \\
\hline 19 Apr 2018 & Sumangnyeong (uphill) - Keunmokjae (uphill) - Eunsinamgol (valley) - Eunsinchi (pass) - forest road \\
\hline 19 Jun 2018 & $\begin{array}{l}\text { Ujeonmaeul (Village) - Pibawi (stone) - Hwangseoksanseong (Fortress) - Hwangseoksanseong nammun (gate) - summit of } \\
\text { Hwangseoksan Mt. }\end{array}$ \\
\hline 20 Jun 2018 & forest road - Eunsinamgol (valley) - Eunsinchi (pass) - forest road \\
\hline $31 \mathrm{Jul} 2018$ & Sorosoryuji (tractional pond) - heliport \\
\hline 1 Aug 2018 & Hwangseoksa (temple) - forest road - heliport \\
\hline
\end{tabular}

\section{표본수집 및 동정}

조사지의 관속식물에 대한 분포증거를 확보하기 위하 여 조사지역 내에 관찰되는 식물 중 꽃, 열매 또는 포자 등 의 생식기관이 있는 개체만을 대상으로 채집을 수행하였 으며, 채집된 모든 식물은 채집번호를 각각 부여하였고, 일차종발생정보의 확보를 위한 GPS정보를 기록하였다. 채집품은 압착.건조 후 표본으로 제작하여 한남대학교 자 연사박물관(HNHM), 국립수목원 $(\mathrm{KH})$, 국립생물자원관 $(\mathrm{KB})$, 한국한의학연구원(KIOM)에 각각 보관하였다.

식물의 동정은 Lee $(1980,2003)$, Lee $(1996,2006)$ 등의 도 감류와 Park (2007)의 식물지 등을 이용하였으며, 확증표 본을 바탕으로 확인된 분류군만을 목록에 포함시켰다.

\section{식물목록작성}

관속식물의 목록 작성은 Engler의 분류체계(Melchior, 1964)에 따라 작성되었고, 과 내에서는 알파벳순으로 속 명과 종소명을 정리하였다. 학명의 사용은 A Synonymic List of Vascular Plants in Korea (Korea National Arboretum and The Plant Taxonomic Society of Korea, 2007)와 Checklist of Vascular Plants in Korea (Korea National Arboretum, 2017) 에 따랐다. 작성된 식물목록(Appendix 1)을 기초로 한국특 산식물은 Chung et al. (2017)에 의하였고, 식물구계학적 특
정식물종은 Floristic Target Species (FT species) in Korea (National Institute of Ecology, 2018)에 따라 파악하였다. 희 귀식물은 Rare Plants Data Book in Korea (Korea National Arboretum, 2008)를, 외래식물은 Checklist of Alien Plants in Korea (Korea National Arboretum, 2019)를 참고하여 작성하 였으며, 재배 또는 식재종은 목록에 따로 표기하였다. 또 한 식물의 유용도 분석은 Lee (1976)에 준하였고, 식물목 록(Appendix 1)에 표기하였다.

\section{결과 및 고찰}

\section{관속식물상}

본 조사지역에서 밝혀진 관속식물상은 금원산 81과 197 속 294종 10 아종 18 변종 3품종의 325분류군, 기백산 93과 226 속 301종 12 아종 28변종 4품종의 345분류군, 황석산 95 과 273 속 387 종 11 아종 30 변종 6 품종의 434 분류군, 거망산 74 과 148 속 187 종 3 아종 15 변종 3 품종의 208 분류군이며, 전체적으로는 도합 107과 346속 583종 14아종 46변종 6품 종으로 총 649분류군이다(Table 2, Appendix 1). 이는 양치 식물 12 과 17 속 29 종 1 변종의 30 분류군(4.6\%), 나자식물 3 과 8 속 8 종의 8 분류군 $(1.2 \%)$, 피자식물의 쌍자엽식물 81 과 259 속 440 종 14 아종 37 변종 4 품종의 495 분류군(76.3\%), 단 
Table 2. Comparison of floras in this investigation with those in previous ones.

\begin{tabular}{lccccccc}
\hline \hline \multicolumn{1}{c}{ Flora } & Family & Genus & Species & Subspecies & Variety & Forma & *Total \\
\hline Geumwonsan Mt. & 81 & 197 & 294 & 10 & 18 & 3 & 325 \\
Gibaeksan Mt. & 93 & 226 & 301 & 12 & 28 & 4 & 345 \\
Hwangsuksan Mt. & 95 & 273 & 387 & 11 & 30 & 6 & 434 \\
Geomangsan Mt. & 74 & 148 & 187 & 3 & 15 & 3 & 208 \\
\hline \multicolumn{1}{c}{ Total } & 107 & 346 & 583 & 14 & 46 & 6 & 649 \\
\hline
\end{tabular}

*Species and infraspecific taxa only

Table 3. Summary of the floristics of Gibaeksan Mt. county park and its neighboring mountains.

\begin{tabular}{lcccccccc}
\hline \hline \multicolumn{1}{c}{ Taxa } & Family & Genus & Species & Subspecies & Variety & Forma & *Total & Ratio (\%) \\
\hline Pteridophyta & 12 & 17 & 29 & 0 & 1 & 0 & 30 & 4.6 \\
Gymnospermae & 3 & 8 & 8 & 0 & 0 & 0 & 8 & 1.2 \\
Angiospermae & 92 & 321 & 546 & 14 & 45 & 6 & 611 & 94.2 \\
Dicotyledons & 81 & 259 & 440 & 14 & 37 & 4 & 495 & 76.3 \\
Monocotyledons & 11 & 62 & 106 & 0 & 8 & 2 & 116 & 17.9 \\
\hline \multicolumn{1}{c}{ Total } & 107 & 346 & 583 & 14 & 46 & 6 & 649 & 100 \\
\hline
\end{tabular}

*Species and infraspecific taxa only

Table 4. List of the major families in Gibaeksan Mt. county park and its neighboring mountains.

\begin{tabular}{lccccc}
\hline \hline \multicolumn{1}{c}{ Family } & Total (No.) & Ratio (\%) & Family & Total (No.) & Ratio (\%) \\
\hline Compositae & 66 & 10.17 & Lamiaceae & 24 & 3.70 \\
Rosaceae & 38 & 5.86 & Ranunculaceae & 22 & 3.39 \\
Poaceae & 36 & 5.55 & Cyperaceae & 22 & 3.39 \\
Liliaceae & 33 & 5.08 & Polygonaceae & 18 & 2.77 \\
Fabaceae & 30 & 4.62 & Apiaceae & 18 & 2.77 \\
\hline
\end{tabular}

자엽식물 11 과 62 속 106 종 8 변종 2품종의 116분류군 (17.9\%)으로 구성되며(Table 3), 한반도 전체 관속식물 4,881분류군(Korea National Arboretum and The Plant Taxonomic Society of Korea, 2007)의 $13.3 \%$, 경상남도 전체 관속식물 953분류군(Oh et al., 2010)의 68.1\%에 해당된다.

식물상 종조성의 주요 10 개과는 국화과가 66 분류군 $(10.2 \%)$ 으로 가장 많았고, 장미과 38 분류군(5.9\%), 벼과 36 분류군(5.6\%), 백합과 33 분류군(5.1\%), 콩과 30 분류군 (4.6\%), 꿀풀과 24 분류군 $(3.7 \%)$, 미나리아재비과 22분류군 (3.4\%), 사초과 22분류군 $(3.4 \%)$ 순이었으며, 마디풀과와 산형과가 18 분류군 $(2.8 \%)$ 으로 가장 적었다. 전체 종조성 에 대한 이들 상위 10 개과가 차지하는 비율은 $47.3 \%$ 로서 약 $1 / 2$ 에 해당된다(Table 4).

본 조사지역의 전체적인 식생은 온대림의 대표수종인 졸참나무, 굴참나무, 신갈나무 등이 우세하나, 개비자나 무, 노각나무, 사람주나무, 대팻집나무 등이 자주 출현하 는 온대 남부의 식생을 보인다.

식물상 우수지역으로서 금원산은 희귀식물인 덩굴꽃
마리와 뻐꾹나리, 식물구계학적 특정식물 IV등급에 해당 되는 가는잎개고사리와 가지괭이눈, III등급종인 너도바 람꽃, 노각나무 등이 생육하고 있는 유안청계곡 인근의 동사면 지역, 기백산은 특산식물인 물들메나무와 금마타 리, 식물구계학적 특정식물 III등급인 긴병꽃풀, 인가목조 팝나무, 시닥나무 등이 확인된 수망령에서 용추계곡까지 의 지역, 거망산은 너도바람꽃, 나도개감채, 뀡의다리아 재비 등이 자생하며, 해발 $800 \mathrm{~m}$ 이상부터 개비자나무 군 락이 출현하는 은신골 지역, 황석산은 돌양지꽃, 천마 등 이 분포하며, 깊은 계곡이 있어 식물상이 비교적 다양하 게 나타나는 황석산성 일원이 본 조사지역의 산지들에서 식물상이 우수한 지역에 해당된다.

\section{선행연구와 비교}

선행연구와 비교함에 있어 기백산은 Lee (1986)와 Kim (2011), 금원산은 Kwon et al. (1997), Seok et al. (2003), Park et al. (2007), Oh et al. (2008)의 조사연구가 있다. 또한 Lee and Oh (1986)는 기백산과 아울러 백운산과 황석산을 조 
사한 바 있으나 식물목록은 산지 구별 없이 관찰된 전체 목록만을 제시하고 있어 단순 비교가 어려운 실정이다. 또한 상기 언급한 선행연구들은 모두 증거표본에 대한 언 급이 없을 뿐만 아니라, 특정 또는 특산식물 등 주요 종으 로 언급된 분류군 중 식물지리학적으로 본 지역의 분포가 의심되는 분류군이 목록에 다수 제시되어 있어 신뢰하기 어렵다. 예로서 특산식물인 히어리에 대하여 Kwon et al. (1997)과 Seok et al. (2003)은 금원산에 분포한다고 언급하 였다. 그러나 Oh et al. (2016)에 따르면 경남지역에서 히어 리는 남해, 하동, 산청지역에서만 그 생육이 확인된 바 있 다. 산림청 지정 희귀식물이자 특산식물인 도깨비부채, 환경부 지정 멸종위기식물이자 특산식물인 자주솜대가 Park et al. (2007)에 의해 금원산 분포가 언급되었지만 도 깨비부채는 경기도와 강원도(Kim, 2007b)에, 자주솜대는 설악산, 소백산, 덕유산, 지리산 등의 백두대간 고봉 $(\mathrm{Oh}$,
2007)에 생육하는 식물로 알려져 있다. 또한 큰천남성은 Seok et al. (2003)과 Oh et al. (2008)에 의해 금원산 분포가 보고된 바 있으나, 본 종은 한반도 남부에 분포하는 난대 성 식물로서 오동정 가능성이 높다. 다만 Seok et al. (2003) 에 의해 보고된 금원산 620 분류군, Kim (2011)에 의한 기 백산 722 분류군과 본 조사에서 금원산과 기백산에서 확 인된 이들 식물 493 분류군과는 많은 차이를 보인다. 그러 나 상기 보고들은 앞서 언급한 바와 같이 증거표본에 대 한 정보가 제시되어 있지 않아 그 실체를 확인할 수 없기 때문에 분류군 수에 대한 비교는 무의미 하다. 다만 본 조 사가 생식기관이 있는 개체만을 대상으로 채집된 확증표 본을 바탕으로 목록이 작성되었고, 조사지역에 생육하고 있을지라도 조사당시 생식기관이 없는 개체는 채집대상 에서 제외되었기 때문이기도 하다. 추후 조사경로와 시기 를 달리하여 보완 조사를 수행한다면 본 산지의 산세로

Table 5. List of the endemic plants in Gibaeksan Mt.county park and its neighboring mountains.

\begin{tabular}{|c|c|c|c|c|c|}
\hline Family name & Taxa & 1 & 2 & 3 & 4 \\
\hline Pinaceae & Abies koreana E. H. Wilson 구상나무 (식) & $\bigcirc$ & - & - & - \\
\hline Ulmaceae & Celtis edulis Nakai 노랑팽나무 & - & - & $\bigcirc$ & - \\
\hline Moraceae & Broussonetia $\times$ hanjiana M. Kim 닥나무 (식) & - & $\bigcirc$ & $\bigcirc$ & - \\
\hline Ranunculaceae & Aconitum austrokoreense Koidz. 세뿔투구꽃 & - & - & $\bigcirc$ & - \\
\hline Ranunculaceae & Aconitum pseudolaeve Nakai 진범 & - & $\bigcirc$ & - & - \\
\hline Ranunculaceae & Clematis brachyura Maxim. 외대으아리 & - & $\bigcirc$ & - & - \\
\hline Ranunculaceae & Clematis trichotoma Nakai 할미밀망 & $\bigcirc$ & $\bigcirc$ & $\bigcirc$ & - \\
\hline Ranunculaceae & Thalictrum actaeifolium Siebold \& Zucc. 은뀡의다리 & $\bigcirc$ & $\bigcirc$ & $\bigcirc$ & $\bigcirc$ \\
\hline Aristolochiaceae & Asarum patens (Yamaki) M. Kim \& S. So 금오족도리풀 & $\bigcirc$ & - & $\bigcirc$ & - \\
\hline Theaceae & Stewartia koreana Nakai ex Rehder 노각나무 & $\bigcirc$ & - & $\bigcirc$ & $\bigcirc$ \\
\hline Rosaceae & Rubus coreamus Miq. var. concolor Nakai ex J. Y. Yang 청 복분자딸기 & - & $\bigcirc$ & - & - \\
\hline Fabaceae & Vicia hirticalycina Nakai 나래완두 & $\bigcirc$ & - & - & - \\
\hline Apiaceae & Angelica reflexa B. Y. Lee 강활 & $\bigcirc$ & $\bigcirc$ & $\bigcirc$ & - \\
\hline Oleaceae & Abeliophyllum distichum Nakai 미선나무 (식) & $\bigcirc$ & - & - & - \\
\hline Oleaceae & Fraxinus chiisanensis Nakai 물들메나무 & - & $\bigcirc$ & - & $\bigcirc$ \\
\hline Lamiaceae & Ajuga spectabilis Nakai 자란초 & - & - & - & $\bigcirc$ \\
\hline Scrophulariaceae & Paulownia coreana Uyeki 오동나무 & $\bigcirc$ & - & $\bigcirc$ & - \\
\hline Caprifoliaceae & Weigela subsessilis (Nakai) L. H. Bailey 병꽃나무 & $\bigcirc$ & $\bigcirc$ & $\bigcirc$ & $\bigcirc$ \\
\hline Valerianaceae & Patrinia saniculifolia Hemsl. 금마타리 & - & $\bigcirc$ & - & - \\
\hline Asteraceae & Aster koraiensis Nakai 벌개미취 & $\bigcirc$ & - & - & - \\
\hline Asteraceae & Cirsium setidens (Dunn) Nakai 고려엉겅퀴 & $\bigcirc$ & $\bigcirc$ & $\bigcirc$ & - \\
\hline Asteraceae & Crepidiastrum koidzumianum (Kitam.) J. H. Pak \& Kawano 지리고들빼기 & - & $\bigcirc$ & - & - \\
\hline Liliaceae & Heloniopsis koreana Fuse, N. S. Lee \& M. N. Tamura 처녀치마 & $\bigcirc$ & - & - & - \\
\hline Liliaceae & Hemerocallis hakuunensis Nakai 백운산원추리 & $\bigcirc$ & $\bigcirc$ & - & - \\
\hline Iridaceae & Iris koreana Nakai 노랑붓꽃 (식) & - & - & $\bigcirc$ & - \\
\hline
\end{tabular}

1, Geumwonsan Mt.; 2, Gibaeksan Mt.; 3, Hwangseoksan Mt.; 4, Geomangsan Mt. 
보아 분류군 수는 다소 증가할 수 있을 것으로 판단된다.

한편, 기존연구와 비교시 본 연구를 통해 새롭게 확인된 분류군은 긴꽃고사리삼, 노랑팽나무, 세뿔투구꽃, 애기고 추나물, 금오족도리풀 등 111 분류군이며, 이들은 소산식 물목록의 일련번호 앞에 ^로 표시하였다(Appendix 1).

\section{한국특산식물}

본 조사지에서 생육이 확인된 한국특산식물은 진범, 외 대으아리, 할미밀망 등 25 분류군이며, 이들 중 구상나무, 미선나무, 닥나무, 노랑붓꽃의 4 분류군은 금원산자연휴 양림에 식재된 것이다(Table 5). 상기 식재된 4분류군을 제 외하면 본 지역에 자생하는 특산식물은 전체 한국특산식 물의 약 $3.23 \%$ 에 해당된다.

한편 확인된 특산식물 중 노랑팽나무는 중부지방의 길 가, 숲, 계곡과 사면에 생육하는 수종으로 알려져 있으며 (Kim, 2007a), 본 조사에서는 황석산성 주변에서 개박달나 무, 신갈나무, 산오리나무, 팥배나무, 대팻집나무와 혼생 하고 있었고, 물들메나무는 수망령과 금원산 용추계곡 주 변을 비롯하여 거망산의 동사면 계곡부에서도 생육을 확 인하였다. 또한 자란초는 거망산의 은신치에서 신갈나무, 산뽕나무, 당단풍나무, 멸가치, 조릿대 등과 함께 우점하 고 있었으며, 노각나무는 금원산, 황석산, 거망산의 산지 계곡주변 또는 그늘진 습한 곳에서 비교적 흔히 관찰되었 고, 진범은 기백산의 정상부 능선과 동북사면의 등산로에 서 다수의 개체가 확인되었다. 조사지역 내 확인된 특산 식물 자생지는 대체적으로 양호하나, 등산로 또는 임도 주변에서 확인된 특산식물은 답압, 불법채취 등에 의한 피해가 우려되며, 금원산 동사면 지역은 위락시설 등이 추가 조성되고 있으므로 자생지의 주변 식생에 대한 관리 방안을 고려할 필요가 있다.

\section{희귀 및 멸종위기식물}

조사지역의 산림청 지정 희귀식물은 총 18 분류군이 확 인되었으며(Table 6), 보존 등급이 높은 critically endangered species $(\mathrm{CR})$ 에서 vulnerable (VU)까지의 등급에 해당되는 종은 총 6 분류군이다. 이들 중 멸종위기종 $(\mathrm{CR})$ 은 목련, 미 선나무, 노랑붓꽃의 3 분류군이 있으나 모두 식재된 것이
며, 취약종(VU)은 세뿔투구꽃, 왜박주가리, 천마의 3 분류 군이며, 위기종(EN)은 발견되지 않았다(Table 6). 이들 중 세뿔투구꽃은 경상도와 지리산, 전남 백운산 등의 주로 우리나라 남부지역에 자생하는 특산식물(Korea Forest Service and Korea National Arboretum, 2016; Lim et al., 2017) 로 본 조사지에서는 황석산 남사면 해발 $600 \mathrm{~m}$ 이상의 등 산로 주변에서 큰꽃으아리, 다래, 개옻나무, 짝자래나무 등과 함께 생육하고 있었다. 그러나 자생지 주변에서 확 인된 개체 수는 많지 않고, 인근 사찰 및 수련원의 방문객 또는 등산객의 왕래가 빈번하여 이들에 의한 훼손이 예상 되며, 세뿔투구꽃의 개체군 보전을 위한 주기적인 모니터 링이 필요할 것으로 판단된다. 왜박주가리는 기백산 동사 면의 고학리에서 늘밭고개로 가는 등산로에서 확인되었 으나 개체 수가 적고, 등산로 주변에 생육하고 있어 제초 및 탐방객 유입 등에 의한 자생지 훼손이 우려되기 때문에 적절한 조치가 요구된다. 또한 천마는 황석산 서사면의 피 바위 주변에서 소수 개체가 발견되었으며, 주위에는 함박 꽃나무, 대팻집나무, 얇은잎고광나무, 노루오줌 등이 생육 하고 있었으나 등산로 주변에 생육하고 있어 답압 및 불법 채취 등과 같은 인위적 간섭에 의한 피해가 우려되므로 이 에 대한 보전 및 관리방안이 필요할 것으로 판단된다. 한 편 환경부 지정 멸종위기 야생 식물은 미선나무, 노랑붓꽃, 세뿔투구꽃의 3 분류군이며, 모두 2 등급에 해당된다.

\section{식물구계학적 특정식물종}

식물구계학적 특정식물종은 자연환경 평가를 위한 식 물군으로 국내 주요종의 보존 우선순위를 결정하는 데 이 용할 수 있으며, 이들을 등급화하여 특정한 지역공간 내 분포현황을 통해 자연환경의 우수성 정도 및 상대적 우열 을 파악하는 데 이용하고자 선정한 목록이다 $(\mathrm{Kim}, 2000$; National Institute of Ecology, 2018). 조사지역의 식물구계학 적 특정식물종은 총 107 분류군이며, 이들을 등급별로 구 분해 보면 $\mathrm{V}$ 등급에는 노랑팽나무와 세뿔투구꽃을 비롯 한 5 분류군이 있으나, 이들 중 목련, 미선나무, 노랑붓꽃 의 3 분류군은 식재된 것이며, IV등급에는 가는잎개고사 리, 가지괭이눈, 꽃싸리, 파란여로, 왕김의털의 5 분류군, III등급에는 식재된 구상나무, 낭아초, 탱자나무, 단풍나

Table 6. List of the rare and endangered plants in Gibaeksan Mt. county park and its neighboring mountains.

\begin{tabular}{llc}
\hline \hline \multicolumn{1}{c}{ Family name } & \multicolumn{1}{c}{ Taxa } & Criteria \\
\hline Magnoliaceae & Magnolia kobus DC. 목련 (식) & CR \\
Oleaceae & Abeliophyllum distichum Nakai 미선나무 (식) & CR \\
Iridaceae & Iris koreana Nakai 노랑붓꽃 (식) & CR \\
Ranunculaceae & Aconitum austrokoreense Koidz. 세뿔투구꽃 & VU \\
Apocynaceae & Tylophora floribunda Miq. 왜박주가리 & VU \\
Orchidaceae & Gastrodia elata Blume 천마 & VU \\
\hline
\end{tabular}

$\overline{\mathrm{CR}}$, critically endangered species; $\mathrm{VU}$, vulnerable. 
Table 7. The list of floristic regional indicator plants (designated by the National Institute Ecology) in Gibaeksan Mt. county park and its neighboring mountains.

\begin{tabular}{|c|c|c|}
\hline Family name & Taxa & Grade \\
\hline Ulmaceae & Celtis edulis Nakai 노랑팽나무 & $\mathrm{V}$ \\
\hline Magnoliaceae & Magnolia kobus DC. 목련 (식) & $\mathrm{V}$ \\
\hline Ranunculaceae & Aconitum austrokoreense Koidz. 세뿔투구꽃 & $\mathrm{V}$ \\
\hline Oleaceae & Abeliophyllum distichum Nakai 미선나무 (식) & $\mathrm{V}$ \\
\hline Iridaceae & Iris koreana Nakai 노랑붓꽃 (식) & $\mathrm{V}$ \\
\hline Athyriaceae & Athyrium iseanum Rosenst. 가는잎개고사리 & IV \\
\hline Saxifragaceae & Chrysosplenium ramosum Maxim. 가지괭이눈 & IV \\
\hline Fabaceae & Campylotropis macrocarpa (Bunge) Rehder 꽃싸리 & IV \\
\hline Liliaceae & Veratrum maackii Regel var. parviflorum (Maxim. ex Miq.) H. Hara 파란여로 & IV \\
\hline Poaceae & Festuca rubra L. 왕김의털 & IV \\
\hline Pinaceae & Abies koreana E. H. Wilson 구상나무 (식) & III \\
\hline Betulaceae & Betula chinensis Maxim. 개박달나무 & III \\
\hline Betulaceae & Betula davurica Pall. 물박달나무 & III \\
\hline Betulaceae & Betula schmidtii Regel 박달나무 & III \\
\hline Ranunculaceae & Actaea asiatica H. Hara 노루삼 & III \\
\hline Ranunculaceae & Clematis brachyura Maxim. 외대으아리 & III \\
\hline Ranunculaceae & Eranthis stellata Maxim. 너도바람꽃 & III \\
\hline Actinidiaceae & Actinidia kolomikta (Maxim. \& Rupr.) Maxim. 쥐다래 & III \\
\hline Theaceae & Stewartia koreana Nakai ex Rehder 노각나무 & III \\
\hline Papaveraceae & Dicentra spectabilis (L.) Lem. 금낭화 & III \\
\hline Hydrangeaceae & Philadelphus pekinensis Rupr. 애기고광나무 & III \\
\hline Grossulariaceae & Ribes mandshuricum (Maxim.) Kom. 까치 밥나무 & III \\
\hline Grossulariaceae & Ribes maximowiczianum Kom. 명자순 & III \\
\hline Rosaceae & Aruncus dioicus (Walter) Fernald 눈개승마 & III \\
\hline Rosaceae & Prunus sargentii Rehder 산벚나무 & III \\
\hline Rosaceae & Sorbaria sorbifolia (L.) A. Braun 쉬땅나무 & III \\
\hline Rosaceae & Spiraea chamaedryfolia L. 인가목조팝나무 & III \\
\hline Rosaceae & Spiraea fritschiana C. K. Schneid. 참조팝나무 & III \\
\hline Fabaceae & Indigofera pseudotinctoria Matsum. 낭아초 (식) & III \\
\hline Rutaceae & Poncirus trifoliata Raf. 탱자나무(식) & III \\
\hline Aceraceae & Acer komarovii Pojark. 시닥나무 & III \\
\hline Aceraceae & Acer palmatum Thunb. 단풍나무 (식) & III \\
\hline Ericaceae & Vaccinium hirtum Thunb. var. koreanum (Nakai) Kitam. 산앵도나무 & III \\
\hline Primulaceae & Lysimachia vulgaris L. var. davurica (Ledeb.) R. Knuth 좁쌀풀 & III \\
\hline Oleaceae & Chionanthus retusus Lindl. \& Paxton 이팝나무 (식) & III \\
\hline Oleaceae & Fraxinus chiisanensis Nakai 물들메나무 & III \\
\hline Valerianaceae & Patrinia saniculifolia Hemsl. 금마타리 & III \\
\hline Liliaceae & Veratrum maackii Regel var. japonicum (Baker) Shimizu 여로 & III \\
\hline Cyperaceae & Carex papulosa Boott 쇠낚시사초 & III \\
\hline
\end{tabular}

무, 이팝나무를 포함하여 29분류군, II등급에는 긴꽃고사 리삼을 비롯한 30 분류군, I등급에는 개비자나무를 비롯한 38 분류군이 각각 확인되었다(Table 7). 이는 조사지 전체 관속식물 대비 약 $16.5 \%$ 에 해당되며, 이들 중 상대적으로 중시되고 있는 III등급 이상의 식물은 총 39분류군이나 식
재종 8 분류군을 제외하면 31 분류군으로서 전체 관속식물 대비 약 $4.77 \%$ 에 해당된다.

이들 중 가지괭이눈은 주로 강원도에 분포하는 식물로 잎은 대생하고, 꽃받침은 퍼지며, 녹색이고, 심피는 컵형 인 특징을 가지는데 본 조사에서 금원산 북사면의 창선리 
계곡과 동사면의 유안청 계곡에서 많은 군락이 확인되었 다. 꽃싸리는 중국, 한국에 자생하는 식물로 국내에서는 경상도 지역에서만 분포가 확인되고 있는 분포 특이식물 이다(Oh et al., 2016). 본 조사에서 꽃싸리는 황석산의 피바 위 주변 등산로에서 확인되었으며, 소나무, 쇠물푸레나 무, 조릿대와 함께 생육하고 있었다. 식물구계학적특정식 물 4등급 이상의 분류군 중 자생하는 종들은 다소 제한된 분포를 보이며, 자생지는 소수의 개체로 구성되므로 추후 자생지 보존과 관리가 필요할 것으로 판단된다. 또한 인 가목조팝나무는 경기, 강원, 충북, 경북, 전북에 분포하며, 줄기는 각이 지고, 화서는 산형상 산방화서이며, 묵은 가 지에 달리고, 수술은 30 개 이상이며, 길이는 꽃잎의 2 배 이 상인 특징이 있는 식물로 본 조사지역에서는 기백산의 정 상부 능선에서 3 개체가 발견되었다. 지금까지 알려진 인 가목조팝나무의 분포역(Oh et al., 2016)을 미루어볼 때 본 자생지는 식물지리학적으로 남방한계분포지로 판단된다.

\section{외래식물}

조사지역에서 확인된 외래식물은 19 과 43 분류군이다 (Table 8). 이는 조사지의 전체 관속식물 대비 약 $6.6 \%$ 로서 우리나라의 평균 귀화율인 $10.3 \%$ (Koh et al., 1995)보다는 낮은 편이다. 조사지역의 외래식물을 분류군 수가 많은 과별로 정리해 보면 국화과가 돼지풀, 망초, 코스모스 등 의 10 분류군, 벼과가 큰이삭풀, 오리새, 큰김의털 등의 5 분 류군, 마디풀과가 애기수영, 묵밭소리쟁이, 소리쟁이 등 4 분류군 순이다. 국내 정착 및 확산여부에 따른 외래식물 분류기준으로 구분해보면 침입외래식물(invasive alien plant)은 29분류군, 잠재침입외래식물(potentially invasive plant)은 9분류군, 사전귀화식물(Arc., archaeophyte)은 5분 류군으로 나타났다. 한편 조사지역 내 생태계교란종은 애 기수영, 돼지풀이 2종으로 확인되었다. 애기수영과 돼지 풀은 황석산 서사면의 사방댐과 임도 주변부에서 발견되 었다. 확인된 위치와 생태계교란종의 개체군 크기로 보아

Table 8. List of the alien plants in Gibaeksan Mt. county park and its neighboring mountains.

\begin{tabular}{|c|c|c|c|}
\hline Family & Species & Classification & $\begin{array}{c}\text { Ecosystem } \\
\text { disturbing } \\
\text { species }\end{array}$ \\
\hline Pinaceae & Larix kaempferi (Lamb.) Carrière 일본잎갈나무 (식) & PIP & - \\
\hline Cupressaceae & Chamaecyparis obtusa (Siebold \& Zucc.) Endl. 편백 (식) & PIP & - \\
\hline Cupressaceae & Cryptomeria japonica (Thunb. ex L.f.) D.Don 삼나무 (식) & PIP & - \\
\hline Betulaceae & Alnus firma Siebold \& Zucc. 사방오리 (식) & PIP & - \\
\hline Polygonaceae & Rumex acetosella L. 애기수영 & IAP & $\bigcirc$ \\
\hline Polygonaceae & Rumex conglomeratus Murray 묵밭소리쟁이 & IAP & - \\
\hline Polygonaceae & Rumex crispus L. 소리쟁이 & IAP & - \\
\hline Polygonaceae & Rumex obtusifolius L. 돌소리쟁이 & IAP & - \\
\hline Phytolaccaceae & Phytolacca americana L. 미국자리공 & IAP & - \\
\hline Caryophyllaceae & Cerastium glomeratum Thuill. 유럽점나도나물 & IAP & - \\
\hline Caryophyllaceae & Silene armeria L. 끈끈이대나물 & IAP & - \\
\hline Caryophyllaceae & Stellaria media (L.) Vill. 별꽃 & IAP & - \\
\hline Magnoliaceae & Liriodendron tulipifera L. 백합나무 (식) & PIP & - \\
\hline Saururaceae & Houttuynia cordata Thunb. 약모밀(식) & Arc. & - \\
\hline Brassicaceae & Lepidium apetalum Willd. 다닥냉이 (식) & PIP & - \\
\hline Brassicaceae & Lepidium virginicum L. 콩다닥냉이 & IAP & - \\
\hline Rosaceae & Kerria japonica (L.) DC. 황매화 (식) & PIP & - \\
\hline Rosaceae & Prunus persica (L.) Stokes 복사나무 (식) & Arc. & - \\
\hline Fabaceae & Amorpha fruticosa L. 족제비싸리 & IAP & - \\
\hline Fabaceae & Styphnolobium japonicum (L.) Schott 회화나무 (식) & PIP & - \\
\hline Fabaceae & Trifolium repens L. 토끼풀 & IAP & - \\
\hline Oxalidaceae & Oxalis corniculata L. 괭이밥 & Arc. & - \\
\hline Anacardiaceae & Toxicodendron vernicifluum (Stokes) F. A. Barkley 옻나무 & Arc. & - \\
\hline Aceraceae & Acer buergerianum Miq. 중국단풍 (식) & PIP & - \\
\hline
\end{tabular}


Table 8. Continued.

\begin{tabular}{|c|c|c|c|}
\hline Family & Species & Classification & $\begin{array}{c}\text { Ecosystem } \\
\text { disturbing } \\
\text { species }\end{array}$ \\
\hline Aquifoliaceae & Ilex serrata Thunb. 낙상홍(식) & IAP & - \\
\hline Onagraceae & Oenothera biennis L. 달맞이꽃 & IAP & - \\
\hline Solanaceae & Solanum nigrum L. 까마중 & Arc. & - \\
\hline Scrophulariaceae & Veronica polita Fr. 개불알풀 & IAP & - \\
\hline Asteraceae & Ambrosia artemisiifolia L. 돼지풀 & IAP & $\bigcirc$ \\
\hline Asteraceae & Conyza canadensis (L.) Cronquist 망초 & IAP & - \\
\hline Asteraceae & Cosmos bipinnatus Cav. 코스모스 & IAP & - \\
\hline Asteraceae & Erechtites hieraciifolius (L.) Raf. ex DC. 붉은서나물 & IAP & - \\
\hline Asteraceae & Erigeron annuus (L.) Pers. 개망초 & IAP & - \\
\hline Asteraceae & Galinsoga ciliata (Raf.) S. F. Blake 털별꽃아재비 & IAP & - \\
\hline Asteraceae & Galinsoga parviflora Cav. 별꽃아재비 & IAP & - \\
\hline Asteraceae & Rudbeckia bicolor Nutt. 원추천인국 & IAP & - \\
\hline Asteraceae & Rudbeckia laciniata L. 삼잎국화 & IAP & - \\
\hline Asteraceae & Taraxacum officinale F. H. Wigg. 서양민들레 & IAP & - \\
\hline Poaceae & Bromus catharticus Vahl 큰이삭풀 & IAP & - \\
\hline Poaceae & Dactylis glomerata L. 오리새 & IAP & - \\
\hline Poaceae & Festuca arundinacea Schreb. 큰김의털 & IAP & - \\
\hline Poaceae & Lolium perenne L. 호밀풀 & IAP & - \\
\hline Poaceae & Poa pratensis L. 왕포아풀 & IAP & - \\
\hline
\end{tabular}

Table 9. Usage types of vascular plants in Gibaeksan Mt. county park and its neighboring mountains.

\begin{tabular}{ccccccccccc}
\hline \hline & $\mathrm{E}$ & $\mathrm{F}$ & $\mathrm{M}$ & $\mathrm{O}$ & $\mathrm{P}$ & $\mathrm{R}$ & $\mathrm{S}$ & $\mathrm{T}$ \\
\hline Number of taxa & 257 & 8 & 206 & 84 & 220 & 4 & 13 & 23 \\
Ratio (\%) & 39.6 & 1.2 & 31.7 & 12.9 & 33.9 & 0.6 & 2.0 & 3.5 \\
\hline
\end{tabular}

E, edible; F, fiber; M, medicinal; O, ornamental; P, pasture; R, industrial raw material; S, plant dyestuff; T, timber source; U, unknown.

등산객 및 임도 공사에서 종자가 유입되어 발아한 후 정 착하여 군락을 형성한 것으로 판단되며, 이들이 산림 내 에 침입하여 추가적으로 확산되지 않도록 제거 및 관리가 필요할 것으로 판단된다.

\section{용도별 구분}

조사지에서 확인된 관속식물 중 Lee (1976)에 근거한 유 용식물은 500 분류군으로서 전체 관속식물 649 분류군의 $77 \%$ 에 해당된다. 이들을 용도별로 구분해보면 식용식물 257 분류군(39.6\%), 섬유용식물 8 분류군(1.2\%), 약용식물 206분류군(31.7\%), 관상용식물 84 분류군(12.9\%), 목초용 식물 220 분류군(33.9\%), 공업용식물 4 분류군 $(0.6 \%)$, 염료 용식물 13 분류군 $(2.0 \%)$, 목재용식물 23 분류군 $(3.5 \%)$ 이었
다(Table 9). 또한 전체 649분류군 중 238분류군이 2가지 이상의 용도를 가지는 것으로 나타났다(Table 9, Appendix 1).

ORCID: Beom Kyun PARK https://orcid.org/0000-0003-18943296; Dong Chan SON https://orcid.org/0000-0002-67730580; Sung Chul KO https://orcid.org/0000-0002-2181-2652

\section{Acknowledgments}

This study was supported by the Korea National Arboretum (KNA1-1-18, 15-3) and National Institute of Biological Resources (NIBR201801206). 


\section{Conflict of Interest}

The authors declare that there are no conflicts of interest.

\section{Literature Cited}

Chung, G. Y., K. S. Chang, J.-M. Chung, H. J. Choi, W.-K. Paik and J.-O. Hyun. 2017. A checklist of endemic plants on the Korean Peninsula. Korean Journal of Plant Taxonomy 47: 264-288.

Geochang-gun. 2018. Geochang Statistical Yearbook. Geochang, 465 pp. (in Korean)

Hamyang-gun. 2018. Hamyang Statistical Yearbook. Hamyang. 520 pp. (in Korean)

Jeong, Y. J. 2017. The vegetation structure and flora of Mt. Geomangsan in Hamyang. MS thesis, Graduate School Gyeongnam National University of Science and Technology, Jinju, Korea, 42 pp. (in Korean)

Kang, S. O. 1971. New Korea Geography. Saegeulsa, Seoul, 606 pp. (in Korean)

Kim, C. H. 2000. Assessment of natural environment: I. Selection of plant taxa. Korean Journal of Environmental Biology, 18: 163-198.

Kim, J. W. 2011. A study on the relationship between vascular plants and forest structure in Gibaeksan(Mt.). MS thesis, Graduate School Gyeongnam National University of Science and Technology, Jinju, 66 pp. (in Korean)

Kim, M. 2007a. Celtis edulis Nakai. In The Genera of Vascular Plants of Korea. Park, C.-W. (ed.), Academy Publ. Co., Seoul. P. 242.

Kim, Y. D. 2007b. Rodgersia podophylla A. Gray. In The Genera of Vascular Plants of Korea. Park, C.-W. (ed.), Academy Publ. Co., Seoul. P. 522.

Koh, K. S., I. G. Kang, M. H. Suh, J. H. Kim, K. D. Kim, J. H. Kil, H. I. Rhu, D. S. Kong, E. B. Lee and E. S. Chun. 1995. Survey for Ecological Impact by Naturalized Organisms (I): Naturalized Plants. National Institute of Environmental Research, Incheon, 215 pp. (in Korean)

Korea Forest Service and Korea National Arboretum. 2016. Distribution Maps of Vascular Plants in Korea. Korea Forest Service and Korea National Arboretum, Pocheon, 809 pp. (in Korean)

Korea National Arboretum. 2008. Rare Plants Data Book in Korea. Geobook Publishing Co., Seoul, 332 pp. (in Korean)

Korea National Arboretum. 2017. Checklist of Vascular Plants in Korea. Korea National Arboretum, Pocheon, 1,000 pp. (in Korean)

Korea National Arboretum. 2019. Checklist of Alien Plants in
Korea. Dooroo Happy Co., Ltd., Seoul, 225 pp. (in Korean) Korea National Arboretum and The Plant Taxonomic Society of Korea. 2007. A Synonymic List of Vascular Plants in Korea. Korea National Arboretum, Pocheon, 534 pp. (in Korean)

Kwon, Y. C., H. C. An, H. S. Cho and G. C. Choo. 1997. Studies on the woody plants of Keum Won San Recreation Forest. Journal of Agricultural Technology Research Institute (Chinju National University) 10: 31-41.

Lee, T. B. 1976. Vascular plants and their uses in Korea. Bulletin of the Arboretum, Seoul National University 1: 1-137.

Lee, T. B. 1980. Illustrated Flora of Korea. Hyangmunsa, Seoul, 990 pp. (in Korean)

Lee, T. B. 1986. Vegetation of Mt. Paegun of Hamyang. The Report of the Korean Association for Conservation of Nature 24: 61-71. (in Korean)

Lee, T. B. 2003. Coloured Flora of Korea. Vol. I, II. Hyangmunsa, Seoul, Vol. I, 914 pp, Vol. II, 910 pp. (in Korean)

Lee, W.-T. and Y. J. Yim. 1978. Studies on the distribution of vascular plants in the Korean Peninsula. Korean Journal of Plant Taxonomy 8 (Appendix): 1-33. (in Korean)

Lee, W. T. and Y. J. Yim. 2002. Plant Geography with Special Reference to Korea. Kangwon National University Press, Chuncheon, 412 pp. (in Korean)

Lee, Y. N. 1996. Flora of Korea, Gyohaksa, Seoul, 1247 pp. (in Korean)

Lee, Y. N. 2006. New Flora of Korea. Vol. I, II. Gyohaksa, Seoul, Vol. I, 974 pp, Vol. II. 885 pp. (in Korean)

Lee, Y. N. and Y. J. Oh. 1986. An investigation for flora on Mt. Paegun, Mt. Kipaeg and Hwangsǒk mountain. The Report of the Korean Association for Conservation of Nature, 24: 4160. (in Korean)

Lim, C. E., T. K. Noh and C.-W. Park. 2017. Aconitum austroKoreense Koidz. In Flora of Korea. Vol. 2a. Magnoliidae. Flora of Korea Editorial Committee (ed.), National Institute of Biological Resources, Incheon. Pp. 49-50.

Melchior, H. 1964. An Engler's Syllabus der pflanzenfamilien. Band II. Gebrüder Borntraeger, Berlin, 666 pp.

National Institute of Ecology. 2018. Floristic Target Species (FT species) in Korea. National Institute of Ecology, Seocheon, 728 pp. (in Korean)

National Institute of Environmental Research. 2012. 2012 The Investigation to Find the Good Area of Ecological Landscape. National Institute of Environmental Research, Incheon. Pp. 541-674. (in Korean)

Oh, B. U. 2007. Smilacina bicolor Nakai. In The Genera of Vascular Plants of Korea. Park, C.-W. (ed.), Academy Publ. Co., Seoul. P. 1313.

Oh, B. U., D. G. Jo, S. C. Ko, H. T. Im, G. Y. Chung, C. G. Jang 
and S. H. Kang. 2010. Distribution Maps of Vascular Plants of Korean Peninsula. VII. South Province (Gyeongsangnam-do) and Ulleung-do Province. Korean Forest Service, Daejeon and Korean National Arboretum, Pocheon, 776 pp. (in Korean)

Oh, B. U., S. C. Ko, S. H. Kang, W. K. Paik, K. O. Yoo, H. T. Im, C. G. Jang, G. Y. Chung, B. H. Choi, H. J. Choi, Y. M. Lee, C. H. Shin, K. Choi, J. S. Han, S. H. Park, H. J. Kim, K. S. Chang, J. C. Yang, S. Y. Jung, C. H. Lee, S. H. Oh and D. G. Jo. 2016. Distribution Maps of Vascular Plants in Korea. Korea National Arboretum, Pocheon, 809 pp.

Oh, H.-K., H.-T. Shin and M.-S. Beon. 2008. Investigation of vascular plants in the Eco-Arboretum Site, Mt. Gumwon. Korean Journal of Plant Resources 21: 117-127.

Oh, H.-K., S.-C. Kim and J.-H. You. 2015. Study on flora distributed around Mt. Hwangseok, Gyeongsangnam-do for selecting the ecological and landscape conservation area. Journal of Environmental Impact Assessment 24: 51-65.
Park, C. W. 2007. The Genera of Vascular Plants of Korea. Academy Publishing Co., Seoul, 1482 pp.

Park, J. H., J. T. Im, G. H. Gu, M. K. Cho, H. R. Jung, J. H. Lee and H. S. Moon. 2007. Species composition of forest vegetation in the Mt. Geumwon recreational forest. Korean Journal of Environmental Agriculture 26(2): 149-158.

Seok, J. C., O. R. Kim and G. C. Choo. 2003. Vascular plants of Keumwonsan area. Journal of Agricultural Technology Research Institute (Chinju National University) 16: 91-113.

Shin, H. C. 2015. Vegetation structure and dynamics of northwest slope in Hwangseoksan (Mt.). MS thesis, Graduate School Gyeongnam National University of Science and Technology, Jinju, 42 pp.

Yim, Y. J. and T. Kira. 1975. Distribution of forest vegetation and climate in the Korean Peninsula: I. Distribution of some indices of thermal climate. Japanese Journal of Ecology 25: 7788.

\section{기백산군립공원과 인접산지의 관속식물상 \\ 박범균 ${ }^{1,2}$ - 손동찬 ${ }^{2}$ - 고성철* \\ 1 한남대학교 생명·나노과학대학 생명시스템과학과 \\ 2국립수목원 산림생물다양성연구과}

적 요: 경상남도 거창군과 함양군의 경계에 위치하고 있는 기백산군립공원과 인접산지의 관속식물상을 2011년 4월부터 9월까지, 2012년 7월, 2015년 4월부터 2018년 8월까지 총 46회에 걸쳐 조사하였다. 그 결과, 본 조사 지역의 관속식물은 107과 346속 583종 14아종 46변종 6품종의 총 649분류군으로 조사되었으며, 25 분류군의 한국특산식물과 18 분류군의 희귀식물이 확인되었다. 식물구계학적 특정식물종은 식재종을 포함하 여 $\mathrm{V}$ 등급에 5 분류군, IV등급에 5분류군, III등급에 29분류군, II등급에 30분류군, I등급에 38분류군이 각각 발 견되었으며, 외래식물은 43 분류군이 확인되었다. 조사된 전체 식물 중 500 분류군이 자원식물이었으며, 이를 용도별로 구분하면 식용식물 257분류군, 약용식물 206분류군, 목초용식물 220분류군, 관상용식물 84 분류군 등이었다. 조사지의 식물상은 한반도 식물구계의 남부아구에 속한다.

주요어: 식물상, 기백산군립공원, 금원산, 기백산, 황석산, 거망산 
Appendix 1. List of the vascular plants of Gibaeksan Mt. county park and its neighboring mountains.

\section{Equisetaceae 속새과}

1. Equisetum arvense L.쇠뜨기 E, P [1, 2, 3]

Geochang-gun (Sangcheon-ri, Geumwonsan Mt.), 110518092[HNHM]; Hamyang-gun (Sangwon-ri, Gibaeksan Mt.), 151016-001 [HNHM]; Hamyang-gun (Sangwon-ri, Hwangseoksan Mt.), 150423-059[HNHM]

Ophioglossaceae 고사리삼과

$\star 2$. Botrychium strictum Underw. 긴꽃고사리삼U[4] Geomangsan Mt., 180619-187 [KB]

3. Sceptridium ternatum (Thunb.) Sw. 고사리삼 $\mathrm{M}[1,2,3]$ Geochang-gun (Sangcheon-ri, Geumwonsan Mt.), 110830067[HNHM]; Hamyang-gun (Sangwon-ri, Gibaeksan Mt.), 150918-012 [KB]; Hamyang-gun (Bongjeon-ri, Hwangseoksan Mt.), 151022-090[HNHM]

Osmundaceae 고비과

4. Osmunda cinnamomea L 뀡고비 U [1] Geochang-gun (Changseon-ri, Geumwonsan Mt.), 110519103[HNHM]

5. Osmunda japonica Thunb.고비E, M[2] Geochang-gun (Gohak-ri, Gibaeksan Mt.), 150709-005[KB]

Dennstaedtiaceae잔고사리과

6. Dennstaedtia hirsuta (Sw.) Mett. ex Miq. 잔고사리U[4] Geomangsan Mt., 180619-183 [KB]

7. Dennstaedtia wilfordii (T. Moore) Christ 황고사리U[3, 4] Hamyang-gun (Hwangsan-ri, Hwangseoksan Mt.), 150604 039 [HNHM]; Geomangsan Mt., 180731-168 [KB]

8. Pteridium aquilinum (L.) Kuhn var. latiusculum(Desv.) Underw. ex Hell. 고사리E, M[2, 3]

Geochang-gun (Gohak-ri, Gibaeksan Mt.), 150812-195[KB];

Hamyang-gun (Bongjeon-ri, Hwangseoksan Mt.), 151022-074 [HNHM]

\section{Aspleniaceae 꼬리고사리과}

9. Asplenium incisum Thunb. 꼬리고사리U[3]

Hamyang-gun (Hwangsan-ri, Hwangseoksan Mt.), 150604$006[\mathrm{HNHM}]$

Thelypteridaceae 처녀고사리과

10. Thelypteris japonica (Baker) Ching 지 네고사리U[1, 2, 3] Hamyang-gun (Sangwon-ri, Geumwonsan Mt.), 110716$050[\mathrm{HNHM}]$; Geochang-gun (Sangcheon-ri, Gibaeksan Mt.), 151015-059 [KB]; Hwangseoksan, 170525-058[KB]

\section{Woodsiaceae 우드풀과}

11. Woodsia polystichoides D. C. Eaton 우드풀 U[2, 4]

Hamyang-gun (Sangwon-ri, Gibaeksan Mt.), 150918-001[KB]; Geomangsan Mt., 180731-092 [KB]

Onocleaceae 야산고비과

12. Matteuccia struthiopteris (L.) Tod. 청나래고사리E[3] Hwangseoksan Mt., 170525-054 [KB]

Athyriaceae 개고사리과

13. Athyrium brevifrons Nakai ex Kitag. 참새발고사리E, M[2, 4] Geochang-gun (Gohak-ri, Gibaeksan Mt.), 150709-115[KB]; Geomangsan Mt., 180619-066 [KB]

$\star$ 14. Athyrium iseanum Rosenst. 가는잎개고사리U[1, 2, 4] Geochang-gun (Sangcheon-ri, Geumwonsan Mt.), 110830007[HNHM]; Hamyang-gun (Sangwon-ri, Gibaeksan Mt.), 151016-021 [KB]; Geomangsan Mt., 180619-153[KB]

15. Athyrium niponicum (Mett.) Hance 개고사리 E[2, 3, 4] Hamyang-gun (Sangwon-ri, Gibaeksan Mt.), 150917-063[KB]; Hamyang-gun (Bongjeon-ri, Hwangseoksan Mt.), 151022-007 [HNHM]; Geomangsan Mt., 180619-147[KB]

16. Asplenium yokoscense (Franch. \& Sav.) Christ 뱀고사리E[1,3]
Geochang-gun (Sangcheon-ri, Geumwonsan Mt.), 110830055[HNHM]; Hamyang-gun (Sangwon-ri, Hwangseoksan Mt.), 150813-069 [HNHM]

$\star$ 17. Cornopteris crenulatoserrulata(Makino) Nakai 응달고사리 $\mathrm{U}[1]$

Geochang-gun (Changseon-ri, Geumwonsan Mt.), 110519-032[HNHM]

18. Deparia conilii (Franch. \& Sav.) M. Kato 좀진고사리U[1, 3, 4] Geochang-gun (Sangcheon-ri, Geumwonsan Mt.), 110830-

124[HNHM]; Hamyang-gun (Bongjeon-ri, Hwangseoksan Mt.), 151022-044 [HNHM]; Geomangsan, 180619-071[KB]

19. Deparia japonica (Thunb.) M. Kato 진고사리U[4] Geomangsan Mt., 180731-187 [KB]

$\star 20$. Deparia pycnosora (Christ) M. Kato 털고사리U[2] Geochang-gun (Gohak-ri, Gibaeksan Mt.), 150812-115[KB]

Dryopteridaceae 관중과

21. Dryopteris chinensis (Baker) Koidz. 가는잎족제비고사리U[1, 3, 4] Geochang-gun (Changseon-ri, Geumwonsan Mt.), 110519107[HNHM]; Hamyang-gun (Hwangsan-ri, Hwangseoksan Mt.), 150604-025 [HNHM]; Geomangsan Mt., 180619-180[KB]

22. Dryopteris crassirhizoma Nakai 관중 E, M [1] Geochang-gun (Sangcheon-ri, Geumwonsan Mt.), 110830114 [HNHM]

23. Dryopteris lacera (Thunb.) Kuntze 비늘고사리U[2, 3, 4] Geochang-gun (Sangcheon-ri, Gibaeksan Mt.), 151015082[KB]; Hamyang-gun (Hwangsan-ri, Hwangseoksan Mt.), 150604-205 [HNHM]; Geomangsan Mt., 180619-063[KB]

24. Dryopteris setosa (Thunb.) Akasawa 산족제비고사리U[2, 3, 4] Hamyang-gun (Sangwon-ri, Gibaeksan Mt.), 150918-107[KB]; Hamyang-gun (Bongjeon-ri, Hwangseoksan Mt.), 151022-032 [HNHM]; Geomangsan Mt., 180418-076[KB]

25. Dryopteris uniformis (Makino) Makino 곰비늘고사리U[4] Geomangsan Mt.,180619-079 [KB]

26. Dryopteris varia (L.) Kuntze 족제비고사리U[3] Hwangseoksan Mt., 180619-192 [KB]

27. Polystichum tripteron (Kunze) C. Presl 십자고사리U[1, 2, 3, 4] Geochang-gun (Changseon-ri, Geumwonsan Mt.), 110519126[HNHM]; Geochang-gun (Sangcheon-ri, Gibaeksan Mt.), 151015-081 [KB]; Hamyang-gun (Bongjeon-ri, Hwangseoksan Mt.), 150710-120[HNHM]; Geomangsan Mt., 180619-150 [KB]

Davalliaceae 넉줄고사리과

28. Davallia mariesii T. Moore ex Baker 넉줄고사리O[2] Geochang-gun (Gohak-ri, Gibaeksan Mt.), 150709-010[KB]

Polypodiaceae 고란초과

29. Lepisorus ussuriensis (Regel \& Maack) Ching 산일엽초U[2, 4] Hamyang-gun (Sangwon-ri, Gibaeksan Mt.), 150917106[HNHM]; Geomangsan Mt., 180419-033 [KB]

30. Selliguea hastata (Thunb.) Fraser-Jenk. 고란초 M[1] Geochang-gun (Sangcheon-ri, Geumwonsan Mt.), 110518-141[HNHM]

Pinaceae 소나무과

31. Abies koreana E. H. Wilson 구상나무(식)O, T[1] Geochang-gun (Sangcheon-ri, Geumwonsan Mt.), 110515001 [HNHM]

32. Larix kaempferi (Lamb.) Carriere 일본잎갈나무(식)T[3] Hamyang-gun (Hwangsan-ri, Hwangseoksan Mt.), 150604043[HNHM]

33. Pinus densiflora Siebold \& Zucc. 소나무 E, M, O, T[1, 2, 3] Geochang-gun (Wolseong-ri, Geumwonsan Mt.), 110514008 [HNHM]; Geochang-gun (Gohak-ri, Gibaeksan Mt.), 150709-048 [KB]; Hamyang-gun (Bongjeon-ri, Hwangseoksan Mt.), 150710-094[HNHM] 
Appendix 1. Continued.

\section{Cupressaceae 측백나무과}

$\star$ 34. Chamaecyparis obtusa (Siebold \& Zucc.) Endl. 편백 (식) O, $\mathrm{T}$ [3] Hamyang-gun (Bongjeon-ri, Hwangseoksan Mt.), 151022028 [HNHM]

35. Cryptomeria japonica (Thunb. ex L.f.) D. Don 삼나무 (식)T[3] Hamyang-gun (Bongjeon-ri, Hwangseoksan Mt.), 151022127[HNHM]

36. Juniperus rigida Siebold \& Zucc. 노간주나무M, S[1] Geochang-gun (Sangcheon-ri, Geumwonsan Mt.), 110518$109[\mathrm{HNHM}]$

^37. Metasequoia glyptostroboidesHu \& W. C. Cheng 메타세쿼이 아(식)O[3]

Hamyang-gun (Hwangsan-ri, Hwangseoksan Mt.), 151023 050[HNHM]

Cephalotaxaceae 개비자나무과

38. Cephalotaxus harringtonii (Knight ex J. Forbes) K. Koch 개비 자나무 $\mathrm{E}, \mathrm{O}[1,2,3,4]$

Hamyang-gun (Sangwon-ri, Geumwonsan Mt.), 110716097[HNHM]; Hamyang-gun (Sangwon-ri, Gibaeksan Mt.), 150424-012 [KB]; Hamyang-gun (Sangwon-ri, Hwangseoksan Mt.), 150423-047[HNHM]; Geomangsan Mt., 180619-144 [KB]

\section{Juglandaceae 가래나무과}

39. Juglans regia L.호두나무 (식) E [3] Hwangseoksan Mt., 170526-083 [KB]

40. Platycarya strobilacea Siebold \& Zucc. 굴피나무S[3] Hamyang-gun (Hwangsan-ri, Hwangseoksan Mt.), 150604 $020[\mathrm{HNHM}]$

\section{Salicaceae 버드나무과}

41. Salix caprea L.호랑버들 P $[1,3,4]$

Geochang-gun (Sangcheon-ri, Geumwonsan Mt.), 110420 036[HNHM]; Hamyang-gun (Sangwon-ri, Hwangseoksan Mt.), 150423-067 [HNHM]; Geomangsan Mt., 180419-068[KB]

42. Salix gracilistyla Miq. 갯버들 P $[1,3,4]$ Geochang-gun (Wolseong-ri, Geumwonsan Mt.), 110514057[HNHM]; Hamyang-gun (Sangwon-ri, Hwangseoksan Mt.), 150423-051 [HNHM]; Geomangsan Mt., 180418-096[KB]

43. Salix pierotii Miq. 버드나무 P [1]

Geochang-gun (Wolseong-ri, Geumwonsan Mt.), 110514059[HNHM]

Betulaceae 자작나무과

44. Alnus firma Siebold \& Zucc. 사방오리S[1, 3]

Geochang-gun (Sangcheon-ri, Geumwonsan Mt.), 110518187[HNHM]; Hamyang-gun (Sangwon-ri, Hwangseoksan Mt.), 150423-008 [HNHM]

45. Alnus incana (L.) Moench subsp. hirsuta(Turcz. ex Spach)

Á.Löve \& D.Löve물오리나무 S [1, 2, 3]

Geochang-gun (Wolseong-ri, Geumwonsan Mt.), 110514039 [HNHM]; Hamyang-gun (Sangwon-ri, Gibaeksan Mt.), 151016-040 [KB]; Hamyang-gun (Hwangsan-ri, Hwangseoksan Mt.), 150604-040[HNHM]

46. Betula chinensis Maxim.개박달나무 T [3]

Hwangseoksan Mt., 170526-001 [KB]

47. Betula davurica Pall.물박달나무 M, T [1, 2, 3]

Geochang-gun (Sangcheon-ri, Geumwonsan Mt.), 110519-

032[HNHM]; Geochang-gun (Sangcheon-ri, Gibaeksan Mt.),

151015-041 [KB]; Hamyang-gun (Bongjeon-ri, Hwangseoksan Mt.), 151022-019[HNHM]

48. Betula schmidtii Regel박달나무 T [1, 2, 3, 4]

Geochang-gun (Sangcheon-ri, Geumwonsan Mt.), 110518 -
126[HNHM]; Geochang-gun (Sangcheon-ri, Gibaeksan Mt.), 151015-038 [KB]; Hamyang-gun (Bongjeon-ri, Hwangseoksan Mt.), 150710-058[HNHM]; Geomangsan Mt., 180418-051 [KB]

49. Carpinus cordata Blume까치박달 P, T [1, 2, 3, 4] Geochang-gun (Sangcheon-ri, Geumwonsan Mt.), 110518063[HNHM]; Hamyang-gun (Sangwon-ri, Gibaeksan Mt.), 150424-055 [KB]; Hwangseoksan Mt., 170713-023[KB]; Geomangsan Mt., 180619-010 [KB]

50. Carpinus laxiflora (Siebold \& Zucc.) Blume 서어나무O, S[2, 3, 4]

Hamyang-gun (Sangwon-ri, Gibaeksan Mt.), 150605-045[KB]; Hamyang-gun (Sangwon-ri, Hwangseoksan Mt.), 150813-086 [HNHM]; Geomangsan Mt, 180731-143[KB]

51. Carpinus tschonoskii (Siebold \& Zucc.) Maxim. 개서어나무O, $\mathrm{S}[1,2]$ Geochang-gun (Changseon-ri, Geumwonsan Mt.), 110519-138 [HNHM]; Hamyang-gun (Sangwon-ri, Gibaeksan Mt.), 150605176 [HNHM]

52. Corylus heterophylla Fisch. ex Trautv. 난티잎개암나무E, M, P[1, 2] Geochang-gun (Sangcheon-ri, Geumwonsan Mt.), 110830162[HNHM]; Hamyang-gun (Sangwon-ri, Gibaeksan Mt.), 150918-109 [KB]

53. Corylus sieboldiana Blume 참개암나무 $\mathrm{E}, \mathrm{M}[1,3]$ Geochang-gun (Sangcheon-ri, Geumwonsan Mt.), 110830134[HNHM]; Hwangseoksan Mt. ,170713-066 [KB]

54. Corylus sieboldiana Blume var. mandshurica(Maxim. \& Rupr.) C. K. Schneid.물개암나무 E, M [1, 2, 4]

Geochang-gun (Sangcheon-ri, Geumwonsan Mt.), 110830127[HNHM]; Geochang-gun (Gohak-ri, Gibaeksan Mt.), 150709-139 [KB]; Geomangsan Mt., 180619-073[KB]

Fagaceae 참나무과

55. Castanea crenata Siebold \& Zucc. 밤나무 (식)E, M, S, T[1, 2, 3] Geochang-gun (Sangcheon-ri, Geumwonsan Mt.), 110604039[HNHM]; Hamyang-gun (Sangwon-ri, Gibaeksan Mt.), 150605-141 [KB]; Hamyang-gun (Hwangsan-ri, Hwangseoksan Mt.), 150604-104[HNHM]

56. Quercus acutissima Carruth. 상수리나무(식)E, M, P, T[3, 4] Hwangseoksan Mt., 180801-100 [KB]; Geomangsan Mt., 180731-130[KB]

57. Quercus aliena Blume갈참나무 E, P, T [2, 3] Geochang-gun (Gohak-ri, Gibaeksan Mt.), 150709-096[KB]; Hamyang-gun (Hwangsan-ri, Hwangseoksan Mt.), 150423-002 [HNHM]

58. Quercus mongolica Fisch. ex Ledeb. 신갈나무E, P, T[1, 2, 3] Geochang-gun (Sangcheon-ri,. Geumwonsan Mt), 110514046[HNHM]; Geochang-gun (Gohak-ri, Gibaeksan Mt.), 150709-137 [KB]; Hamyang-gun (Bongjeon-ri, Hwangseoksan Mt.), 150710-005[HNHM]

59. Quercus serrata Murray졸참나무 E, M, P, T [1, 2, 3] Geochang-gun (Wolseong-ri, Geumwonsan Mt.), 110514028[HNHM]; Geochang-gun (Gohak-ri, Gibaeksan Mt.), 150709-155 [KB]; Hamyang-gun (Sangwon-ri, Hwangseoksan Mt.), 150813-123[HNHM]

60. Quercus variabilis Blume 굴참나무E, M, R, T[1, 3] Geochang-gun (Sangcheon-ri, Geumwonsan Mt.), 110518059[HNHM]; Hamyang-gun (Bongjeon-ri, Hwangseoksan Mt.), 150710-053 [HNHM]

Ulmaceae 느릅나무과

$\star 61$. Celtis edulis Nakai노랑팽나무 E [3]

Hwangseoksan Mt.-160921-048 [KB] 
Appendix 1. Continued.

62. Celtis sinensis Pers. 팽나무 E, M, P [2, 3]

Hamyang-gun (Sangwon-ri, Gibaeksan Mt.), 150605 -

001 [HNHM]; Hwangseoksan Mt., 170525-001 [KB]

63. Ulmus davidiana Planch. 당느릅나무 E, F, M, T [1]

Geochang-gun (Sangcheon-ri, Geumwonsan Mt.), 110518-

$182[\mathrm{HNHM}]$

64. Ulmus davidiana Planch. var. japonica(Rehder) Nakai 느릅나

무E, F, M, T[1, 3]

Geochang-gun (Changseon-ri, Geumwonsan Mt.), 110519-

$110[\mathrm{HNHM}]$; Hamyang-gun (Hwangsan-ri, Hwangseoksan Mt.), 150604-060 [HNHM]

65. Zelkova serrata (Thunb.) Makino 느티나무E, T[2, 3, 4] Hamyang-gun (Sangwon-ri, Gibaeksan Mt.), 150424-025[KB]; Hwangseoksan Mt., 180619-100 [KB]; Geomangsan Mt., 180731-164 [KB]

\section{Moraceae 뽕나무과}

$\star$ 66. Broussonetia $\times$ hanjiana M. Kim닥나무 (식) E, F, M [2, 3$]$ Hamyang-gun (Sangwon-ri, Gibaeksan Mt.), 150605-229[KB]; Hwangseoksan Mt., 170525-168 [KB]

67. Morus australis Poir.산뽕나무 E, M, P, S [1, 2, 3, 4] Geochang-gun (Sangcheon-ri, Geumwonsan Mt.), 110518041 [HNHM]; Hamyang-gun (Sangwon-ri, Gibaeksan Mt.), 150605-140 [HNHM]; Hamyang-gun (Sangwon-ri, Hwangseoksan Mt.), 150423-056[HNHM]; Geomangsan Mt., 180619-058 [KB]

Cannabaceae 삼과

68. Humulus scandens (Lour.) Merr. 환삼덩굴E, $\mathrm{M}, \mathrm{P}[1,3]$ Geochang-gun (Wolseong-ri, Geumwonsan Mt.), 110918089[HNHM]; Hamyang-gun (Bongjeon-ri, Hwangseoksan Mt.), 151022-136 [HNHM]

\section{Urticaceae 쐐기풀과}

69. Boehmeria japonica (L.f.) Miq. 왜모시풀F[1, 2, 4] Geochang-gun (Sangcheon-ri, Geumwonsan Mt.), 110830051 [HNHM]; Geochang-gun (Gohak-ri, Gibaeksan Mt.), 150812-125 [KB]; Geomang Mt., 180731-055[KB]

70. Boehmeria platanifolia (Franch. \& Sav.) C. H. Wright 개모시풀F[1, 3] Hamyang-gun (Sangwon-ri, Geumwonsan Mt.), 110716-

126[HNHM]; Hamyang-gun (Bongjeon-ri, Hwangseoksan Mt.), 151022-027 [HNHM]

71. Boehmeria spicata (Thunb.) Thunb. 좀깨잎나무 $\mathrm{F}[1,2,3,4]$ Geochang-gun (Sangcheon-ri, Geumwonsan Mt.), 110830046[HNHM]; Geochang-gun (Gohak-ri, Gibaeksan Mt.), 150709-084 [KB]; Hamyang-gun (Sangwon-ri, Hwangseoksan Mt.), 150813-007[HNHM]; Geomangsan Mt., 180731-067 [KB]

$\star 72$. Boehmeria tricuspis (Hance) Makino var. unicuspis Makino ex Ohwi 풀거북꼬리E, F[3] Hamyang-gun (Sangwon-ri, Hwangseoksan Mt.), 150813$003[\mathrm{HNHM}]$

73. Pilea peploides (Gaudich.) Hook. \& Arn. 물통이 U[2] Hamyang-gun (Sangwon-ri, Gibaeksan Mt.), 150917-010[KB]

$\star$ 74. Pilea pumila (L.) A. Gray 모시물통이P[1,3] Geochang-gun (Sangcheon-ri, Geumwonsan Mt.), 110830107[HNHM]; Hamyang-gun (Bongjeon-ri, Hwangseoksan Mt.), 151022-128 [HNHM]

75. Pilea pumila (L.) A. Gray var. hamaoi(Makino) C. J. Chen 큰물 통이 $\mathrm{U}[2,3]$

Hamyang-gun (Sangwon-ri, Gibaeksan Mt.), 151016-016[KB];

Hwangseoksan Mt., 160921-028 [KB]

Santalaceae 단향과

76. Thesium chinense Turcz.제비꿀 M [1, 3]
Geochang-gun (Sangcheon-ri, Geumwonsan Mt.), 110518-

$118[\mathrm{HNHM}]$; Hamyang-gun (Sangwon-ri, Hwangseoksan Mt.), 150423-043 [HNHM]

Viscaceae 겨우살이과

77. Viscum album L. var. coloratum (Kom.) Ohwi겨우살이 M [4] Geomangsan Mt., 180419-054 [KB]

Polygonaceae 마디풀과

78. Fallopia ciliinervis (Nakai) K. Hammer 나도하수오U[2] Hamyang-gun (Sangwon-ri, Gibaeksan Mt.), 150917-033[KB]

$\star$ 79. Fallopia dentatoalata (F. Schmidt) Holub 큰닭의 덩굴U[3] Hamyang-gun (Sangwon-ri, Hwangseoksan Mt.), 150813$062[\mathrm{HNHM}]$

80. Fallopia dumetorum (L.) Holub 닭의덩굴U[2] Hamyang-gun (Sangwon-ri, Gibaeksan Mt.), 150918-130[KB]

81. Persicaria dissitiflora (Hemsl.) H. Gross ex T. Mori 가시여뀌U[1, $2,3]$

Hamyang-gun (Sangwon-ri, Geumwonsan Mt.), 110716129 [HNHM]; Geochang-gun (Gohak-ri, Gibaeksan Mt.), 150812-006 [KB]; Hwangseoksan Mt., 160922-076[KB]

82. Persicaria filiformis (Thunb.) Nakai ex T. Mori 이삭여뀌 $\mathrm{M}[1,3,4]$ Geochang-gun (Sangcheon-ri, Geumwonsan Mt.), 110830012[HNHM]; Hamyang-gun (Sangwon-ri, Hwangseoksan Mt.), 150813-058 [HNHM]; Geomangsan Mt., 180731-167[KB]

83. Persicaria lapathifolia (L.) Delarbre 흰여뀌 U[1, 2] Geochang-gun (Wolseong-ri, Geumwonsan Mt.), 110918091 [HNHM]; Geochang-gun (Gohak-ri, Gibaeksan Mt.), 150709-068 [KB]

84. Persicaria longiseta (Bruijn) Kitag. 개여뀌 M[1, 2, 3, 4] Geochang-gun (Sangcheon-ri, Geumwonsan Mt.), 110830153[HNHM]; Geochang-gun (Gohak-ri, Gibaeksan Mt.), 150812-097 [KB]; Hamyang-gun (Bongjeon-ri, Hwangseoksan Mt.), 151022-139[HNHM]; Geomangsan Mt., 180731-046 [KB]

85. Persicaria nepalensis (Meisn.) H. Gross 산여뀌 $\mathrm{P}[1,2,3]$ Geochang-gun (Sangcheon-ri, Geumwonsan Mt.), 110830071 [HNHM]; Geochang-gun (Gohak-ri, Gibaeksan Mt.), 150812-005 [KB]; Hamyang-gun (Sangwon-ri, Hwangseoksan Mt.), 150813-107[HNHM]

86. Persicaria perfoliata (L.) H. Gross 며느리배꼽E[1, 2] Geochang-gun (Sangcheon-ri, Geumwonsan Mt.), 10830193[HNHM]; Geochang-gun (Gohak-ri, Gibaeksan Mt.), 150709-026 [KB]

87. Persicaria posumbu (Buch.-Ham. ex D. Don) H. Gross 장대여뀌 $\mathrm{U}[2,3]$

Geochang-gun (Gohak-ri, Gibaeksan Mt.), 150812-014[KB]; Hamyang-gun (Sangwon-ri, Hwangseoksan Mt.), 150813-014 [HNHM]

88. Persicaria sagittata (L.) H. Gross 미꾸리낚시 $\mathrm{P}[2,3]$ Geochang-gun (Gohak-ri, Gibaeksan Mt.), 150709-107[KB]; Hwangseoksan Mt., 160922-017 [KB]

89. Persicaria senticosa (Meisn.) H. Gross ex Nakai 며느리밑씻개 E, $\mathrm{P}[1,2,3,4]$

Geochang-gun (Sangcheon-ri, Geumwonsan Mt.), 110830280[HNHM]; Geochang-gun (Gohak-ri, Gibaeksan Mt.), 150812-123 [KB]; Hamyang-gun (Sangwon-ri, Hwangseoksan Mt.), 150813-106[HNHM]; Geomangsan Mt., 180731-161 [KB]

90. Persicaria thunbergii (Siebold \& Zucc.) H. Gross 고마리 P $[1,2,3]$ Geochang-gun (Sangcheon-ri, Geumwonsan Mt.), 110830088[HNHM]; Hamyang-gun (Sangwon-ri, Gibaeksan Mt.), 150918-046 [KB]; Hamyang-gun (Bongjeon-ri, Hwangseoksan Mt.), $151022-026[\mathrm{~KB}]$ 
Appendix 1. Continued.

91. Rumex acetosa $\mathrm{L}$. 수영 $\mathrm{M}[1,2,3]$

Geochang-gun (Sangcheon-ri, Geumwonsan Mt.), 110518-

072[HNHM]; Hamyang-gun (Sangwon-ri, Gibaeksan Mt.), 150424-046 [KB]; Hamyang-gun (Sangwon-ri, Hwangseoksan Mt.), 150423-036[HNHM]

92. Rumex acetosella $\mathrm{L}$. 애기수영 $\mathrm{E}$ [3]

Hamyang-gun (Bongjeon-ri, Hwangseoksan Mt.), 150710085[HNHM]

93. Rumex conglomeratus Murray 묵밭소리쟁이 $\mathrm{M}, \mathrm{P}[4]$ Geomangsan Mt., 180619-032 [KB]

94. Rumex crispus L.소리쟁이 E, M, P [2, 3]

Geochang-gun (Gohak-ri, Gibaeksan Mt.), 150709-120[KB];

Hwangseoksan Mt., 170525-173 [KB]

95. Rumex obtusifolius L.돌소리쟁이 U [1]

Hamyang-gun (Sangwon-ri, Geumwonsan Mt.), 110716-

$091[\mathrm{HNHM}]$

\section{Phytolaccaceae 자리공과}

96. Phytolacca americana L. 미국자리공 U [2, 3, 4]

Geochang-gun (Gohak-ri, Gibaeksan Mt.), 150709-036[KB]; Hamyang-gun (Sangwon-ri, Hwangseoksan Mt.), 150813-037 [HNHM]; Geomangsan Mt., 180731-204[KB]

\section{Molluginaceae 석류풀과}

97. Mollugo pentaphylla L.석류풀 U [3]

Hamyang-gun (Sangwon-ri, Hwangseoksan Mt.), 150813040 [HNHM]

Caryophyllaceae 석죽과

98. Arenaria serpyllifolia L. 벼룩이자리E[3]

Hamyang-gun (Sangwon-ri, Hwangseoksan Mt.), 150423002 [HNHM]

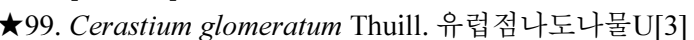
Hamyang-gun (Sangwon-ri, Hwangseoksan Mt.), 150423 048[HNHM]

100. Cerastium holosteoides Fr. var. hallaisanense(Nakai) Mizush. 점나도나물 $\mathrm{E}[1,2]$

Geochang-gun (Wolseong-ri, Geumwonsan Mt.), 110514035[HNHM]; Hamyang-gun (Sangwon-ri, Gibaeksan Mt.), 150424-030 [KB]

101. Dianthus chinensis L.패랭이꽃 M [3] Hwangseoksan Mt., 180801-093 [KB]

102. Pseudostellaria heterophylla(Miq.) Pax 개별꽃 E, M[1, 3, 4] Geochang-gun (Sangcheon-ri, Geumwonsan Mt.), 110420028 [HNHM]; Hamyang-gun (Sangwon-ri, Hwangseoksan Mt.), 150423-027 [HNHM]; Geomangsan Mt., 180419-047[KB]

103. Pseudostellaria palibiniana(Takeda) Ohwi 큰개별 꽃 $\mathrm{E}[2,3,4]$ Hamyang-gun (Sangwon-ri, Gibaeksan Mt.), 150424-089[KB]; Hwangseoksan Mt., 170525-123 [KB]; Geomangsan Mt., 180418-021 [KB]

$\star$ 104. Silene armeria L.근끈이 대나물 U [3, 4] Hamyang-gun (Bongjeon-ri, Hwangseoksan Mt.), 150519035[HNHM]; Geomangsan Mt., 180731-047 [KB]

105. Silene firma Siebold \& Zucc. 장구채 E, M, P[1, 3, 4] Geochang-gun (Sangcheon-ri, Geumwonsan Mt.), 110830282[HNHM]; Hamyang-gun (Sangwon-ri, Hwangseoksan Mt.), 150813-111 [HNHM], Geomangsan Mt., 180731-334[KB]

106. Silene seoulensis Nakai가는장구채 P [3, 4]

Hamyang-gun (Bongjeon-ri, Hwangseoksan Mt.), 150710015[HNHM]; Geomangsan Mt., 180731-201 [KB]

107. Stellaria aquatica (L.) Scop. 쇠별꽃 E, M[1, 2, 3] Geochang-gun (Changseon-ri, Geumwonsan Mt.), 110519 098[HNHM]; Hamyang-gun (Sangwon-ri, Gibaeksan Mt.),
150918-157 [KB]; Hamyang-gun( Bongjeon-ri, Hwangseoksan Mt.), 150519-011[HNHM]

108. Stellaria media (L.) Vill. 별꽃 E [2]

Hamyang-gun (Sangwon-ri, Gibaeksan Mt.), 150424-032[KB]

109. Stellaria uliginosa Murray 벼룩나물 $\mathrm{E}[1,2]$

Geochang-gun (Sangcheon-ri, Geumwonsan Mt.), 110518 -

038[HNHM]; Hamyang-gun (Sangwon-ri, Gibaeksan Mt.), 150424-053 [KB]

Chenopodiaceae 명아주과

110. Chenopodium album L. var. centrorubrum Makino 명아주 E, P[2]

Hamyang-gun (Sangwon-ri, Gibaeksan Mt.), 150918-187[KB]

Amaranthaceae 비름과

111. Achyranthes bidentata Blume var. japonicaMiq. 쇠무릎 E, M, P[2, 3] Geochang-gun (Gohak-ri, Gibaeksan Mt.), 150812-013[KB];

Hamyang-gun (Bongjeon-ri, Hwangseoksan Mt.), 151022-114 [HNHM]

\section{Magnoliaceae 목련과}

$\star$ 112. Liriodendron tulipifera L. 백합나무(식)U[3]

Hwangseoksan Mt., 170526-034 [KB]

113. Magnolia kobus DC.목련M, O[3]

Hwangseoksan Mt.-170525-129 [KIOM]

114. Magnolia sieboldii K. Koch 함박꽃나무O, P[1, 2, 3, 4] Geochang-gun (Sangcheon-ri, Geumwonsan Mt.), 10518083[HNHM]; Hamyang-gun (Sangwon-ri, Gibaeksan Mt.), 150605-026 [KB]; Hwangseoksan Mt., 160921-017[KB]; Geomangsan Mt., 180619-134 [KB]

Schisandraceae 오미자과

115. Schisandra chinensis (Turcz.) Baill. 오미자 (식)E, M[1, 2, 3] Geochang-gun (Sangcheon-ri, Geumwonsan Mt.), 110518047[HNHM]; Geochang-gun (Gohak-ri, Gibaeksan Mt.), 150812-080 [KB]; Hamyang-gun (Bongjeon-ri, Hwangseoksan Mt.), 150710-123[HNHM]

\section{Lauraceae 녹나무과}

116. Lindera erythrocarpa Makino 비목나무O $\mathrm{O} 1,2,3,4]$ Geochang-gun (Sangcheon-ri, Geumwonsan Mt.), 110518107[HNHM]; Hamyang-gun (Sangwon-ri, Gibaeksan Mt.), 150424-080 [KB]; Hamyang-gun (Hwangsan-ri, Hwangseoksan Mt.), 150423-005[HNHM], Geomangsan Mt., 180418-043 [KB]

117. Lindera glauca (Siebold \& Zucc.) Blume 감태나무S[3] Hamyang-gun (Hwangsan-ri, Hwangseoksan Mt.), 150604 046[HNHM]

118. Lindera obtusiloba Blume 생강나무E, M, O[1, 2, 3, 4] Geochang-gun (Sangcheon-ri, Geumwonsan Mt.), 110420003[HNHM]; Geochang-gun (Gohak-ri, GibaeksanMt.),150709176 [KB]; Hamyang-gun (Hwangsan-ri, Hwangseoksan Mt.), 150604-010 [HNHM]; Geomangsan Mt., 180619-028 [KB].

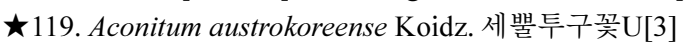
Hamyang-gun (Hwangsan-ri, Hwangseoksan Mt.), 150604195[HNHM]

120. Aconitum jaluense Kom. 투구꽃 M [2, 3]

Hamyang-gun (Sangwon-ri, Gibaeksan Mt.), 150918-020[KB];

Hamyang-gun (Bongjeon-ri, Hwangseoksan Mt.), 151022-006 [HNHM]

121. Aconitum pseudolaeve Nakai 진범 M [2] Geochang-gun (Sangcheon-ri, Gibaeksan Mt.), 151015-025[KB]

122. Actaea asiatica $\mathrm{H}$. Hara노루삼 $\mathrm{U}[1,3,4]$ Geochang-gun (Changseon-ri, Geumwonsan Mt.), 110519 062[HNHM]; Hamyang-gun (Hwangsan-ri, Hwangseoksan Mt.), 150423-025 [HNHM]; Geomangsan Mt., 180731$307[\mathrm{~KB}]$ 
Appendix 1. Continued.

123. Actaea dahurica (Turcz. ex Fisch. \& C. A. Mey.) Franch. 눈빛 승마E, M[3]

Hamyang-gun (Hwangsan-ri, Hwangseoksan Mt.), 150604007[HNHM]

124. Adonis amurensis Regel \& Radde 복수초 $\mathrm{M}, \mathrm{O}[4]$ Geomangsan Mt., 180419-003 [KB]

125. Anemone raddeana Regel꿩 의바람꽃 O [4] Geomangsan Mt., 180419-039 [KB]

126. Caltha palustris L.동의나물 P [4] Geomangsan Mt., 180418-064 [KB]

127. Clematis apiifolia DC.사위질빵 $\mathrm{U}[1,2,3,4]$ Geochang-gun (Sangcheon-ri, Geumwonsan Mt.), 110830194[HNHM]; Geochang-gun (Gohak-ri, Gibaeksan Mt.), 150812-039 [KB]; Hamyang-gun (Sangwon-ri, Hwangseoksan Mt.), 150813-055[HNHM]; Geomangsan Mt., 180731-127 [KB]

128. Clematis brachyura Maxim. 외대으아리 $\mathrm{M}[2]$ Geochang-gun (Gohak-ri, Gibaeksan Mt.), 150709-028[KB]

129. Clematis patens C. Morren \& Decne. 큰꽃으아리O[3] Hamyang-gun (Hwangsan-ri, Hwangseoksan Mt.), 150604181 [HNHM]

130. Clematis terniflora DC. var. mandshurica(Rupr.) Ohwi 으아리 $\mathrm{M}[3]$

Hamyang-gun (Hwangsan-ri, Hwangseoksan Mt.), 150604090[HNHM]

131. Clematis trichotoma Nakai 할미밀 망U[1, 2, 3] Geochang-gun (Sangcheon-ri, Geumwonsan Mt.), 110604011 [HNHM]; Hamyang-gun (Sangwon-ri, Gibaeksan Mt.), 150917-005 [KB]; Hwangseoksan Mt., 170525-190[KB]

132. Eranthis stellata Maxim. 너도바람꽃 $\mathrm{U}[1,4]$ Geochang-gun (Sangcheon-ri, Geumwonsan Mt.), 110420 019 [HNHM]; Geomangsan Mt., 180419-025 [HNHM]

133. Pulsatilla koreana (Y. Yabe ex Nakai) Nakai ex T. Mori 할미 翋 $\mathrm{M}[1,3]$

Geochang-gun (Sangcheon-ri, Geumwonsan Mt.), 110515 041 [HNHM]; Hwangseoksan Mt., 170525-192 [KB]

134. Ranunculus chinensis Bunge 젓가락나물 $\mathrm{U}[2]$ Geochang-gun (Gohak-ri, Gibaeksan Mt.), 150709-044[KB]

135. Ranunculus japonicus Thunb. 미나리아재비 $\mathrm{M}, \mathrm{P}[1,2]$ Geochang-gun (Wolseong-ri, Geumwonsan Mt.), 110514010 [HNHM]; Hamyang-gun (Sangwon-ri, Gibaeksan Mt.), 150424-088 [KB]

136. Ranunculus quelpaertensis (H. Lév.) Nakai 왜젓가락나물U[1]

Geochang-gun (Sangcheon-ri, Geumwonsan Mt.), 110830198 [HNHM]

$\star$ 137. Ranunculus tachiroei Franch. \& Sav. 개구리미나리M[1, 2, 3] Geochang-gun (Sangcheon-ri, Geumwonsan Mt.), 110830066[HNHM]; Geochang-gun (Gohak-ri, Gibaeksan Mt.), 150812-065 [KB]; Hamyang-gun (Sangwon-ri, Hwangseoksan Mt.), 150813-031[HNHM]

138. Thalictrum actaeifolium Siebold \& Zucc. 은꿩 의다리E[1, 2, 3, 4] Hamyang-gun (Sangwon-ri, Geumwonsan Mt.), 110716095[HNHM]; Geochang-gun (Gohak-ri, Gibaeksan Mt.), 150812-015 [KB]; Hamyang-gun (Hwangsan-ri, Hwangseoksa Mt.), 150604-032[HNHM]; Geomangsan Mt., 180731-212 [KB]

139. Thalictrum aquilegifolium $\mathrm{L}$.. var. sibiricumRegel \& Tiling 아 시아꿩의다리 $\mathrm{E}, \mathrm{M}, \mathrm{P}[1,2,3]$

Hamyang-gun (Sangwon-ri, Geumwonsan Mt.), 110716016[HNHM]; Geochang-gun (Gohak-ri, Gibaeksan Mt.), 150812-165 [KB]; Hamyang-gun (Hwangsan-ri, Hwangseoksan Mt.), 151023-080[HNHM]
140. Thalictrum tuberiferum Maxim. 산꿩의다리 $\mathrm{M}[1,4]$ Hamyang-gun (Sangwon-ri, Geumwonsan Mt.), 110716124[HNHM]; Geomangsan Mt., 180619-145 [KB]

Berberidaceae 매자나무과

141. Caulophyllum robustum Maxim. 꿩의다리아재비E, M[4] Geomangsan Mt., 180619-231 [KB]

Lardizabalaceae 으름덩굴과

142. Akebia quinata (Houtt.) Decne. 으름덩굴 M, O, P[2, 3] Hamyang-gun (Sangwon-ri, Gibaeksan Mt.), 150424-001[KB]; Hamyang-gun (Hwangsan-ri, Hwangseoksan Mt.), 150423-018 [HNHM]

\section{Menispermaceae 방기과}

143. Cocculus orbiculatus (L.) DC. 댕댕이덩굴E, M, P[2, 3, 4] Geochang-gun (Gohak-ri, Gibaeksan Mt.), 150812-169[KB]; Hamyang-gun (Sangwon-ri, Hwangseoksan Mt.), 150813-096 [HNHM]; Geomangsan Mt., 180731-008[KB]

144. Menispermum dauricum DC. 새모래덩굴U[1, 3] Geochang-gun (Changseon-ri, Geumwonsan Mt.), 110519 040[HNHM]; Hwangseoksan Mt., 170526-050 [KB]

\section{Saururaceae 삼백초과}

$\star$ 145. Houttuynia cordata Thunb. 약모밀 (식)M[3] Hwangseoksan Mt., 180619-211 [KB]

Chloranthaceae 흘아비꽃대과

146. Chloranthus japonicus Siebold 홀아비꽃대U[2, 3, 4] Geochang-gun (Gohak-ri, Gibaeksan Mt.), 150709-121[KB]; Hamyang-gun (Hwangsan-ri, Hwangseoksan Mt.), 150604-051 [HNHM]; Geomangsan Mt., 180731-313[KB]

Aristolochiaceae 쥐방울덩굴과

$\star 147$. Asarum patens (Yamaki) M. Kim \& S. So 금오족도리풀 $\mathrm{U}[1,3]$

Geochang-gun(Sangcheon-ri, Geumwonsan Mt.), 110514010 [HNHM], Hamyang-gun(Sangwon-ri, Hwangseoksan Mt.), 150423-014 [HNHM]

148. Asarum sieboldii Miq.족도리풀 M [1, 2, 3, 4] Geochang-gun (Sangcheon-ri, Geumwonsan Mt.), 110420018 [HNHM]; Hamyang-gun (Sangwon-ri, Gibaeksan Mt.), 150424-022 [KB]; Hwangseoksan Mt., 170525-010[KB]; Geomangsan Mt., 180419-066 [KB]

\section{Actinidiaceae 다래나무과}

149. Actinidia arguta (Siebold \& Zucc.) Planch. ex Miq. 다래 E, M $[2,3,4]$

Hamyang-gun (Sangwon-ri, Gibaeksan Mt.), 150605-144[KB]; Hamyang-gun (Bongjeon-ri, Hwangseoksan Mt.), 150519-021 [HNHM]; Geomangsan Mt,.180619-110[KB]

150. Actinidia kolomikta (Maxim. \& Rupr.) Maxim. 쥐다래 E[4] Geomangsan Mt., 180731-192 [KB]

Ranunculaceae 미나리아재비과

151. Actinidia polygama (Siebold \& Zucc.) Planch. ex Maxim. 개 다래 $\mathrm{E}, \mathrm{M}[2]$

Geochang-gun (Gohak-ri, Gibaeksan Mt.),150812-049[KB]

Theaceae 차나무과

152. Stewartia koreana Nakai ex Rehder 노각나무O, T[1, 3, 4] Geochang-gun (Sangcheon-ri, Geumwonsan Mt.), 110518127[HNHM]; Hwangseoksan Mt., 160922-022 [KB]; Geomangsan Mt., 180731-179[KB]

Clusiaceae 물레나물과

153. Hypericum ascyron L.물레나물 $\mathrm{E}, \mathrm{O}[2,3,4]$ Geochang-gun (Gohak-ri, Gibaeksan Mt.), 150812-011[KB]; Hamyang-gun (Sangwon-ri, Hwangseoksan Mt.), 150813-112 [HNHM]; Geomangsan Mt., 180731-123[KB] 
Appendix 1. Continued.

154. Hypericum erectum Thunb. 고추나물 $\mathrm{M}, \mathrm{P}[2,3,4]$ Geochang-gun (Gohak-ri, Gibaeksan Mt.), 150812-106[KB]; Hamyang-gun (Bongjeon-ri, Hwangseoksan Mt.), 150710-063 [HNHM]; Geomangsan Mt., 180731-002[KB]

$\star$ 155. Hypericum japonicum Thunb. 애기고추나물 $\mathrm{U}[2]$ Geochang-gun (Gohak-ri, Gibaeksan Mt.), 150812-166[KB]

156. Hypericum laxum (Blume) Koidz. 좀고추나물U[4] Geomangsan Mt., 180731-266 [KB]

Papaveraceae 양귀비과

157. Chelidonium majus L. var. asiaticum(Hara) Ohwi 애기똥풀 $\mathrm{M}[1,2,3]$

Geochang-gun (Sangcheon-ri, Geumwonsan Mt.), 110518006[HNHM]; Hamyang-gun (Sangwon-ri, Gibaeksan Mt.), 150424-051 [KB]; Hamyang-gun (Sangwon-ri, Hwangseoksan Mt.), 150423-075[HNHM]

\section{Fumariaceae 현호색과}

$\star$ 158. Corydalis pauciovulata Ohwi 선괴불주머니U[1, 2, 3$]$ Geochang-gun (Sangcheon-ri, Geumwonsan Mt.), 110830$100[\mathrm{HNHM}]$; Hamyang-gun (Sangwon-ri, Gibaeksan Mt.), 150917-144 [KB]; Hamyang-gun (Bongjeon-ri, Hwangseoksan Mt.), 151022-087[HNHM]

159. Corydalis remota Fisch. ex Maxim. 현호색 $\mathrm{M}[1,2,3,4]$ Geochang-gun (Sangcheon-ri, Geumwonsan Mt.), 110420007[HNHM]; Hamyang-gun (Sangwon-ri, Gibaeksan Mt.), 150424-023 [KB]; Hamyang-gun (Sangwon-ri, Hwangseoksan Mt.), 150423-012[HNHM]; Geomangsan Mt., 180419-002[KB]

160. Corydalis speciosa Maxim. 산괴불주머니U[1, 2, 3, 4] Geochang-gun (Sangcheon-ri, Geumwonsa Mt.), 110420004[HNHM]; Hamyang-gun (Sangwon-ri, Gibaeksan Mt.), 150424-062 [KB]; Hamyang-gun (Sangwon-ri, Hwangseoksan Mt.), 150423-005[HNHM]; Geomangsan Mt., 180418-028 [KB]

161. Corydalis ternata (Nakai) Nakai 들현호색 M[1] Geochang-gun (Sangcheon-ri, Geumwonsan Mt.), 110518037[HNHM]

162. Dicentra spectabilis (L.) Lem. 금낭화 E, O[1] Geochang-gun (Sangcheon-ri, Geumwonsan Mt.), 110604008[HNHM]

\section{Cruciferae 십자화과}

$\star$ 163. Berteroella maximowiczii (Palib.) O. E. Schulz 장대냉이 $\mathrm{E}[2,3]$

Geochang-gun (Gohak-ri, Gibaeksan Mt.), 150709-073[KB]; Hwangseoksan Mt., 170526-015 [KB]

164. Capsella bursa-pastoris (L.) Medicus 냉이 E, M [1, 2, 4] Geochang-gun (Wolseong-ri, Geumwonsan Mt.), 110514068 [HNHM]; Hamyang-gun (Sangwon-ri, Gibaeksan Mt.), 150424-042 [KB]; Geomangsan Mt., 180418-069[KB]

165. Cardamine fallax (O. E. Schulz) Nakai 좁쌀냉이 E[1, 3] Geochang-gun (Sangcheon-ri, Geumwonsan Mt.), 110518074[HNHM]; Hamyang-gun (Sangwon-ri, Hwangseoksan Mt.), 150423-055 [HNHM]

166. Cardamine flexuosa With. 황새냉이 $\mathrm{E}[1,3,4]$ Geochang-gun (Sangcheon-ri, Geumwonsan Mt.), 110420026[HNHM]; Hwangseoksan Mt., 170525-018 [KB]; Geomangsan Mt., 180619-178[KB]

167. Cardamine impatiens L.싸리냉이 E [1, 2, 3, 4] Geochang-gun (Wolseong-ri, Geumwonsan Mt.), 110514031 [HNHM]; Hamyang-gun (Sangwon-ri, Gibaeksan Mt.), 150424-060 [KB]; Hamyang-gun (Hwangsan-ri, Hwangseoksan Mt.), 150604-114[HNHM]; Geomangsan Mt., 180418-037 [KB] 168. Cardamine leucantha (Tausch) O. E. Schulz 미나리냉이E[1, 2, 3, 4]
Geochang-gun (Wolseong-ri, Geumwonsan Mt.), 110514049 [HNHM]; Hamyang-gun (Sangwon-ri, Gibaeksan Mt.), 150605-118 [KB]; Hwangseoksan Mt., 170525-142[KB]; Geomangsan Mt., 180619-057 [KB]

169. Draba nemorosa L.꽃다지 E, M [1, 2, 3, 4] Geochang-gun (Changseon-ri, Geumwonsan Mt.), 10519 056[HNHM]; Hamyang-gun (Sangwon-ri, Gibaeksan Mt.), 150424-036 [KB]; Hamyang-gun (Sangwon-ri, Hwangseoksan Mt.), 150423-003[HNHM]; Geomangsan Mt., 180418-104 [KB]

170. Lepidium apetalum Willd. 다닥냉이(식)E, $\mathrm{M}[2,3]$

Hamyang-gun (Sangwon-ri, Gibaeksan Mt.), 150605-051[KB]; Hwangseoksan Mt., 170525-227 [KB]

171. Lepidium virginicum $\mathrm{L}$. 콩다닥냉이 $\mathrm{U}$ [3]

Hamyang-gun (Hwangsan-ri, Hwangseoksan Mt.), 150604142[HNHM]

172. Rorippa indica (L.) Hiern 개갓냉이E[2] Hamyang-gun (Sangwon-ri, Gibaeksan Mt.), 150605-023[KB]

173. Rorippa palustris (L.) Besser 속속이풀 $\mathrm{E}$ [2]

Hamyang-gun (Sangwon-ri, Gibaeksan Mt.), 150917-095[KB]

174. Arabis glabra L.장대나물 E [1,3]

Geochang-gun (Sangcheon-ri, Geumwonsan Mt.), 110518-

002[HNHM]; Hamyang-gun (Bongjeon-ri, Hwangseoksan Mt.), 150519-033 [HNHM]

\section{Crassulaceae 돌나물과}

175. Hylotelephium erythrostictum(Miq.) H. Ohba 꿩의비름E, M, $\mathrm{P}[2]$

Hamyang-gun (Sangwon-ri, Gibaeksan Mt.), 150917-001[KB]

176. Hylotelephium verticillatum(L.) H. Ohba 세잎꿩의비름U[2] Hamyang-gun (Sangwon-ri, Gibaeksan Mt.), 150917-193[KB]

$\star 177$. Hylotelephium viviparum (Maxim.) H. Ohba 새끼꿩의비름 $\mathrm{O}[2,3]$

Hamyang-gun (Sangwon-ri, Gibaeksan Mt.), 150917-068[KB]; Hamyang-gun (Sangwon-ri, Hwangseoksan Mt.), 150813-063 [HNHM]

178. Phedimus aizoon (L.) 't Hart 가는기린초E[1, 2, 4] Geochang-gun (Sangcheon-ri, Geumwonsan Mt.), 110604$090[\mathrm{HNHM}]$; Hamyang-gun (Sangwon-ri, Gibaeksan Mt.), 150917-154 [KB]; Geomangsan Mt, 180731-222 [KB]

179. Phedimus kamtschaticus (Fisch. \& C. A. Mey.) 't Hart 기린초 E[1, 2, 3] Geochang-gun (Sangcheon-ri, Geumwon Mt.), 110604091 [HNHM]; Hamyang-gun (Sangwon-ri, Gibaeksan Mt.), 150605-155 [KB]; Hamyang-gun (Bongjeon-ri, Hwangseoksan Mt.), 151022-072[HNHM]

180. Sedum bulbiferum Makino말똥비름 U [2, 3] Geochang-gun (Gohak-ri, Gibaeksan Mt.), 150709-161[KB]; Hamyang-gun (Hwangsan-ri, Hwangseoksan Mt.), 150604-013 [HNHM]

181. Sedum polytrichoides Hemsl. 바위채송화U[2] Geochang-gun (Gohak-ri, Gibaeksan Mt.), 150812-209[KB]

182. Sedum sarmentosum Bunge돌나물 E, M [2, 3]

Hamyang-gun (Sangwon-ri, Gibaeksan Mt.), 150605-196[KB]; Hwangseoksan Mt., 170526-064 [KB]

\section{Saxifragaceae 범의귀과}

183. Astilbe chinensis (Maxim.) Franch. \& Sav. 노루오줌M, P[1, 2, $3,4]$

Hamyang-gun (Sangwon-ri, Geumwonsan Mt.), 110716011 [HNHM]; Geochang-gun (Gohak-ri, Gibaeksan Mt.), 150812-091 [KB]; Hamyang-gun (Bongjeon-ri, Hwangseoksan Mt.), 150710-054[HNHM]; Geomangsan Mt., 180731-207 [KB] 184. Astilbe koreana (Kom.) Nakai 숙은노루오줌U[1,3] 
Appendix 1. Continued.

Geochang-gun (Sangcheon-ri, Geumwonsan Mt.), 110830 085[HNHM]; Hamyang-gun (Bongjeon-ri, Hwangseoksan Mt.), 150710-002 [HNHM]

185. Chrysosplenium flagelliferumF. Schmidt 애기괭이눈 $U[1,3]$ Geochang-gun (Sangcheon-ri, Geumwonsan Mt.), 110420$010[\mathrm{HNHM}]$; Hamyang-gun (Sangwon-ri, Hwangseoksan Mt.), 150423-009 [KB]

186. Chrysosplenium grayanum Maxim. 괭이눈 U[4] Geomansan Mt., 180418-081 [KB]

187. Chrysosplenium japonicum (Maxim.) Makino 산괭이눈U[1, 3, 4] Geochang-gun (Sangcheon-ri, Geumwonsan Mt.), 110420033[HNHM]; Hwangseoksan Mt., 170525-124 [KB]; Geomangsan Mt., 180419-031[KB]

$\star$ 188. Chrysosplenium ramosum Maxim. 가지괭이눈 U[1] Geochang-gun (Sangcheon-ri, Geumwonsan Mt.), 110515-015[HNHM]

189. Saxifraga fortunei Hook. 바위떡풀E[1, 2]

Geochang-gun (Sangcheon-ri, Geumwonsan Mt.), 110830016[HNHM]; Hamyang-gun (Sangwon-ri, Gibaeksan Mt.), $150917-028$ [KB]

\section{Hydrangeaceae 수국과}

190. Deutzia glabrata Kom.물참대 O [1, 3, 4] Geochang-gun (Changseon-ri, Geumwonsan Mt.), 110519076[HNHM]; Hwangseoksan Mt., 170525-019 [KB]; Geomangsan Mt., 180619-190[KB]

191. Deutzia parviflora Bunge 말발도리O[1, 2, 3] Geochang-gun (Sangcheon-ri, Geumwonsan Mt.), 110604009 [HNHM]; Geochang-gun (Gohak-ri, Gibaeksan Mt.), 150709-001 [KB]; Hwangseoksan Mt., 160921-088[KB]

192. Deutzia uniflora Shirai매화말발도리 O [1, 2, 3, 4] Geochang-gun (Sangcheon-ri, Geumwonsan Mt.), 110420$002[\mathrm{HNHM}$ ]; Hamyang-gun (Sangwon-ri, Gibaeksan Mt.), 150424-072 [KB]; Hamyang-gun (Sangwon-ri, Hwangseoksan Mt.), 150423-021[HNHM]; Geomangsan Mt., 180418-044 [KB] 193. Hydrangea macrophylla (Thunb.) Ser. subsp. serrata(Thunb.) Makino 산수국 $\mathrm{O}[1,2,3,4]$

Geochang-gun (Sangcheon-ri, Geumwonsan Mt.), 110519010[HNHM]; Geochang-gun (Gohak-ri, Gibaeksan Mt.), 150709-042 [KB]; Hamyang-gun (Bongjeon-ri, Hwangseoksan Mt.), 150710-032[HNHM]; Geomangsan Mt., 180731-186 [KB]

$\star$ 194. Philadelphus pekinensis Rupr. 애기고광나무O[3] Hwangseoksan Mt., 170526-046 [KB]

195. Philadelphus temuifolius Rupr. \& Maxim. 얇은잎고광나무O $[1,2,3,4]$ Geochang-gun (Changseon-ri, Geumwonsan Mt.), 110519166[HNHM]; Hamyang-gun (Sangwon-ri, Gibaeksan Mt.), 150605-068 [KB]; Hamyang-gun (Bongjeon-ri, Hwangseoksan Mt.), 150519-019[HNHM]; Geomangsan Mt., 180731-265 [KB]

\section{Grossulariaceae 까치밥나무과}

196. Ribes fasciculatum Siebold \& Zucc. var. chinenseMaxim. 까마 귀밥나무 $\mathrm{U}[2,4]$

Hamyang-gun (Sangwon-ri, Gibaeksan Mt.), 150424-071[KB]; Geomangsan Mt., 180418-022 [KB]

197. Ribes mandshuricum (Maxim.) Kom. 까치 밥나무E, O[1, 3] Geumwonsan Mt., 160415-004 [KIOM]; Hamyang-gun (Sangwon-ri, Hwangseoksan Mt.), 150423-019[HNHM]

$\star$ 198. Ribes maximowiczianum Kom. 명자순 O[3] Hwangseoksan Mt., 170525-199 [KB]

Rosaceae 장미과

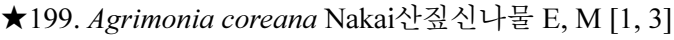
Hamyang-gun (Sangwon-ri, Geumwonsan Mt.), 110716041 [HNHM]; Hamyang-gun (Bongjeon-ri, Hwangseoksan Mt.),

\section{0-029 [HNHM]}

200. Agrimonia pilosa Ledeb.짚신나물 E, M, P [1, 2, 3, 4] Geochang-gun (Sangcheon-ri, Geumwonsan Mt.), 110830118 [HNHM]; Geochang-gun (Gohak-ri, Gibaeksan Mt.), 150709-202 [KB]; Hamyang-gun(Sangwon-ri, Hwangseoksan Mt.), 150813-017[HNHM]; Geomangsan Mt., 180731-076 [KB]

201. Aruncus dioicus (Walter) Fernald 눈개승마U[2, 3] Hamyang-gun (Sangwon-ri, Gibaeksan Mt.), 150918-095[KB]; Hwangseoksan Mt., 160921-077 [KB]

202. Duchesnea indica (Andr.) Focke 뱀딸기 E, M, P[1, 3, 4] Geochang-gun (Wolseong-ri, Geumwonsan Mt.), 110514024[HNHM]; Hamyang-gun (Hwangsan-ri, Hwangseoksan Mt.), 150423-031 [HNHM]; Geomangsan Mt., 180619-031[KB]

203. Geum aleppicum Jacq. 큰뱀무 E, P [1] Hamyang-gun (Sangwon-ri, Geumwonsan Mt.), 110716025 [HNHM]

204. Geum japonicum Thunb. 뱀무E, M, P[1, 2, 4] Geochang-gun (Sangcheon-ri, Geumwonsan Mt.), 110604092[HNHM]; Geochang-gun(Gohak-ri, Gibaeksan Mt.), 150709-049 [KB]; Geomangsan Mt., 180731-005[KB]

$\star 205$. Kerria japonica (L.) DC. 황매화 (식)O[3] Hamyang-gun (Hwangsan-ri, Hwangseoksan Mt.), 150423$011[\mathrm{HNHM}]$

206. Malus baccata Borkh.야광나무 E, O [2, 3]

Hamyang-gun (Sangwon-ri, Gibaeksan Mt.), 150605-101[KB]; Hwangseoksan Mt., 170525-162 [KB]

$\star 207$. Malus mandshurica (Maxim.) Kom. ex Skvortsov 털야광나 무E, $\mathrm{O}[1]$

Geochang-gun (Wolseong-ri, Geumwonsan Mt.), 110514052[HNHM]

208. Malus toringo (Siebold) de Vriese 아그배나무E, O[1, 2, 3, 4] Geochang-gun (Sangcheon-ri, Geumwonsan Mt.), 110518143[HNHM]; Hamyang-gun (Sangwon-ri, Gibaeksan Mt.), 150605-193 [KB]; Hamyang-gun (Bongjeon-ri, Hwangseoksan Mt.), 150519-004[HNHM]; Geomangsan Mt., 180619-121 [KB]

209. Potentilla ancistrifolia Bunge var. dickinsii(Franch. \& Sav.) Koidz. 돌양지꽃 $\mathrm{U}[2,3]$

Hamyang-gun (Sangwon-ri, Gibaeksan Mt.), 150917-015[KB]; Hamyang-gun (Bongjeon-ri, Hwangseoksan Mt.), 150710-043 [HNHM]

$\star 210$. Potentilla chinensis Ser. 딱지꽃 E, M, P[4] Geomangsan Mt., 180731-279 [KB]

211. Potentilla fragarioides $\mathrm{L}$. 양지 꽃 $\mathrm{E}, \mathrm{M}[1,2,3,4]$ Geochang-gun (Sangcheon-ri, Geumwonsan Mt.), 110420027[HNHM]; Hamyang-gun (Sangwon-ri, Gibaeksan Mt.), 150424-015 [KB]; Hamyang-gun (Sangwon-ri, Hwangseoksan Mt.), 150423-026[HNHM]; Geomangsan Mt., 180418-004 [KB]

212. Potentilla freyniana Bornm. 세 잎양지 꽃 E, $\mathrm{P}[1,3,4]$ Geochang-gun (Sangcheon-ri, Geumwonsan Mt.), 110514005 [HNHM]; Hamyang-gun (Hwangsan-ri, Hwangseoksan Mt.), 150423-036 [HNHM]; Geomangsan Mt., 180418-111[KB]

213. Potentilla kleiniana Wight \& Arn. 가락지나물E, P[1, 2, 3, 4] Geochang-gun (Changseon-ri, Geumwonsan Mt.), 110519077[HNHM]; Hamyang-gun (Sangwon-ri, Gibaeksan Mt.), 150605-024 [KB]; Hamyang-gun (Sangwon-ri, Hwangseoksan Mt.), 150813-099[HNHM]; Geomangsan Mt., 180731-247 [KB]

214. Potentilla rosulifera $\mathrm{H}$. Lév. 민눈양지꽃 U[1] Geochang-gun (Sangcheon-ri, Geumwonsan Mt.), 110515-030[HNHM]

$\star 215$. Prunus armeniaca L.살구나무 E, M, O [3] Hamyang-gun (Bongjeon-ri, Hwangseoksan Mt.), 150519-022[HNHM] 
Appendix 1. Continued.

216. Prunus padus L. 귀룽나무 E, M [2]

Hamyang-gun (Sangwon-ri, Gibaeksan Mt.), 150605-152[KB]

217. Prunus persica (L.) Batsch 복사나무E, $\mathrm{P}[1,2,3,4]$ Geochang-gun (Wolseong-ri, Geumwonsan Mt.), 110514075[HNHM]; Hamyang-gun (Sangwon-ri, Gibaeksan Mt.), 150605-149 [KB]; Hamyang-gun (Sangwon-ri, Hwangseoksan Mt.), 150423-068[HNHM]; Geomangsan Mt., 180418-088 [KB]

218. Prunus sargentii Rehder산벚나무 O, P [1] Geumwonsan Mt., 160415-001 [KIOM]

$\star 219$. Prunus serrulata Lindl. var. pubescens(Makino) Nakai 잔털 벚나무E, $\mathrm{O}[3,4]$

Hwangseoksan Mt., 170525-259 [KB]; Geomangsan Mt., 180418-094[KB]

220. Prunus serrulata Lindl. f. spontanea(E. H. Wilson) Chin S. Chang벚나무 E, O [3]

Hwangseoksan Mt., 180619-032 [KB]

221. Rosa multiflora Thunb.찔레꽃 E, M, P [1, 2, 3] Geochang-gun (Sangcheon-ri, Geumwonsan Mt.), 110604028[HNHM], Geochang-gun (Gohak-ri, Gibaeksan Mt.), 150709-064 [KB]; Hamyang-gun (Bongjeon-ri, Hwangseoksan Mt.), 150519-017[HNHM]

222. Rubus coreanus Miq. 복분자딸기 $\mathrm{E}, \mathrm{M}[1,2,4]$ Geochang-gun (Changseon-ri, Geumwonsan Mt.), 110519 075[HNHM]; Hamyang-gun (Sangwon-ri, Gibaeksan Mt.), 150605-012 [KB]; Geomangsan Mt., 180619-101[KB]

$\star 223$. Rubus coreanus Miq. var. concolorNakai ex J. Y. Yang 청복 분자딸기 $\mathrm{E}[2]$

Hamyang-gun (Sangwon-ri, Gibaeksan Mt.), 150605-234[KB]

224. Rubus crataegifolius Bunge 산딸기 $\mathrm{E}, \mathrm{M}, \mathrm{P}[1,3]$ Geochang-gun (Sangcheon-ri, Geumwonsan Mt.), 110604051 [HNHM]; Hamyang-gun (Bongjeon-ri, Hwangseoksan Mt.), 150519-018 [HNHM]

225. Rubus parvifolius L.멍석 딸기 E, M [2, 3, 4] Hamyang-gun (Sangwon-ri, Gibaeksan Mt.), 150605-138[KB]; Hamyang-gun (Bongjeon-ri, Hwangseoksan Mt.), 150519-023 [HNHM]; Geomangsan Mt., 180619-124[KB]

226. Rubus phoenicolasius Maxim. 곰딸기 $\mathrm{E}[2,3,4]$ Geochang-gun (Gohak-ri, Gibaeksan Mt.), 150709-038[KB]; Hamyang-gun (Hwangsan-ri, Hwangseoksan Mt.), 150604-106 [HNHM]; Geomangsan Mt., 180731-060[KB]

227. Rubus pungens Cambess.줄딸기 $\mathrm{E}[1,2,3,4]$ Geochang-gun (Wolseong-ri, Geumwonsan Mt.), 110514012 [HNHM]; Hamyang-gun (Sangwon-ri, Gibaeksan Mt.), 150424-069 [KB]; Hamyang-gun (Sangwon-ri, Hwangseoksan Mt.), 150423-032[HNHM]; Geomangsan Mt., 180418-099 [KB]

228. Sanguisorba officinalis L. 오이풀 M, P[1, 2, 3] Geochang-gun (Sangcheon-ri, Geumwonsan Mt.), 110830172[HNHM]; Geochang-gun (Gohak-ri, Gibaeksan Mt.), 150812-003 [KB]; Hamyang-gun (Hwangsan-ri, Hwangseoksan Mt.), 151023-064[HNHM]

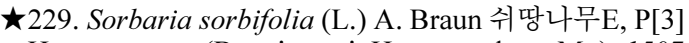
Hamyang-gun (Bongjeon-ri, Hwangseoksan Mt.), 150710 111 [HNHM]

230. Sorbus alnifolia (Siebold \& Zucc.) K. Koch 팥배나무E, P[1] Geochang-gun (Changseon-ri, Geumwonsan Mt.), 110519 094[HNHM]

^231. Spiraea chamaedryfolia L. 인가목조팝나무O[2] Hamyang-gun (Sangwon-ri, Gibaeksan Mt.), 150917-178[KB]

232. Spiraea fritschiana Schneid 참조팝나무 $\mathrm{O}[1,2]$ Geochang-gun (Sangcheon-ri, Geumwonsan Mt.), 110604-
075[HNHM]; Hamyang-gun (Sangwon-ri, Gibaeksan Mt.), 150917-053 [KB]

$\star 233$. Spiraea miyabei Koidz. 덤불조팝나무 O [2] Geochang-gun (Gohak-ri, Gibaeksan Mt.), 150709-065[KB]

234. Spiraea prunifolia Siebold \& Zucc. f. simplicifloraNakai 조팝 나무E, $\mathrm{M}, \mathrm{O}[1,2,3,4]$ Geochang-gun (Sangcheon-ri, Geumwonsan Mt.), 110420015[HNHM]; Hamyang-gun (Sangwon-ri, Gibaeksan Mt.), 150424-033 [KB]; Hamyang-gun (Sangwon-ri, Hwangseoksan Mt.), 150423-061[HNHM]; Geomangsan Mt., 180418-039 [KB]

$\star 235$. Spiraea salicifolia L.꼬리조팝나무 E, M, O [2] Geochang-gun (Gohak-ri, Gibaeksan Mt.), 150709-051[KB]

236. Stephanandra incisa (Thunb.) Zabel 국수나무 $\mathrm{S}[1,2,3,4]$ Geochang-gun (Sangcheon-ri, Geumwonsan Mt.), 110515011 [HNHM]; Hamyang-gun (Sangwon-ri, Gibaeksan Mt.), 150605-121 [KB]; Hamyang-gun (Bongjeon-ri, Hwangseoksan Mt.), 150519-005[HNHM]; Geomangsan Mt., 180731-346 [KB]

Leguminosae 콩과

237. Albizia julibrissin Durazz. 자귀나무M, O, P[3]

Hamyang-gun (Hwangsan-ri, Hwangseoksan Mt.), 150604 004[HNHM]

238. Amorpha fruticosa L.족제 비싸리 P $[1,3]$ Geochang-gun (Sangcheon-ri, Geumwonsan Mt.), 110604068[HNHM]; Hwangseoksan Mt., 170714-026 [KB]

239. Amphicarpaea bracteata (L.) Fernald subsp. edgeworthii(Benth.) H. Ohashi 새콩 E, P [1, 2, 3] Geochang-gun (Sangcheon-ri, Geumwonsan Mt.), 110830001 [HNHM]; Hamyang-gun (Sangwon-ri, Gibaeksan Mt.), 150918-139 [KB]; Hamyang-gun (Hwangsan-ri, Hwangseoksan Mt.), 151023-009[HNHM]

$\star 240$. Campylotropis macrocarpa (Bunge) Rehder 꽃싸리 O, P[3] Hamyang-gun (Bongjeon-ri, Hwangseoksan Mt.), 150710-092 [HNHM]

241. Chamaecrista nomame (Siebold) H. Ohashi 차풀 E, M, P [2, 4] Geochang-gun (Gohak-ri, Gibaeksan Mt.), 150812-062[KB]; Geomangsan Mt., 180731-034 [KB]

Hamyang-gun (Sangwon-ri, Hwangseoksan Mt.), 150423 077[HNHM]; Geomangsan Mt., 180619-198 [KB]

$\star 242$. Crotalaria sessiliflora L. 활나물 U[2] Geochang-gun (Gohak-ri, Gibaeksan Mt.), 150812-093[KB]

243. Glycine $\max$ (L.) Merr. subsp. soja(Siebold \& Zucc.) H. Ohashi돌콩E, $\mathrm{P}[2]$

Hamyang-gun (Sangwon-ri, Gibaeksan Mt.), 150918-140[KB]

244. Hylodesmum oldhamii (Oliv.) H. Ohashi \& R. R. Mill 큰도둑 놈의갈고리 $\mathrm{P}[2,3]$

Hamyang-gun (Sangwon-ri, Gibaeksan Mt.), 150918-166[KB]; Hamyang-gun (Sangwon-ri, Hwangseoksan Mt.),150813-116 [HNHM]

$\star 245$. Hylodesmum podocarpum (DC.) H. Ohashi \& R. R. Mill 개 도둑놈의갈고리U[2]

Geochang-gun (Gohak-ri, Gibaeksan Mt.), 150812-205[KB]

246. Hylodesmum podocarpum (DC.) H. Ohashi \& R. R. Mill subsp. oxyphyllum(DC.) H. Ohashi \& R. R. Mill도둑놈의갈고리 U [2, 3] Geochang-gun (Gohak-ri, Gibaeksan Mt.), 150812-001[KB]; Hamyang-gun (Sangwon-ri, Hwangseoksan Mt.),150813-004 [HNHM]

$\star 247$. Hylodesmum podocarpum (DC.) H. Ohashi \& R. R. Mill var. mandshuricum(Maxim.) H. Ohashi \& R. R. Mill애기도둑놈 의갈고리 U [3]

Hamyang-gun (Sangwon-ri, Hwangseoksan Mt.), 150813115 [HNHM] 
Appendix 1. Continued.

248. Indigofera kirilowii Maxim. ex Palib. 땅비싸리 O[1] Geochang-gun (Sangcheon-ri, Geumwonsan Mt.), 110604093[HNHM]

249. Indigofera pseudotinctoriaMatsum. 낭아초 (식)M, $\mathrm{O}[2,3]$ Geochang-gun (Sangcheon-ri, Gibaeksan Mt.), 151015$069[\mathrm{~KB}]$; Hamyang-gun (Bongjeon-ri, Hwangseoksan Mt.), 151022-129 [HNHM]

250. Lathyrus davidii Hance활량나물 E, M [2, 3] Geochang-gun (Gohak-ri, Gibaeksan Mt.), 150812-136[KB]; Hamyang-gun (Bongjeon-ri, Hwangseoksan Mt.),151022-037 [HNHM]

251. Lespedeza bicolor Turcz. 싸리 O, P [1, 2, 3] Hamyang-gun (Sangwon-ri, Geumwonsan Mt.), 110716087[HNHM]; Geochang-gun (Gohak-ri, Gibaeksan Mt.),150709-070 [KB]; Hwangseoksan Mt., 170713-103[KB]

252. Lespedeza cuneata (Dum.Cours.) G. Don 비수리 P[2, 3] Geochang-gun (Sangcheon-ri, Gibaeksan Mt.), 151015072[KB]; Hamyang-gun (Bongjeon-ri, Hwangseoksan Mt.),151022-133 [HNHM]

253. Lespedeza cyrtobotrya Miq. 참싸리 $\mathrm{F}, \mathrm{O}[1,2,3,4]$ Geochang-gun (Wolseong-ri, Geumwonsan Mt.), 110918050[HNHM]; Hamyang-gun (Sangwon-ri, Gibaeksan Mt.), 150917-281 [KB]; Hamyang-gun (Hwangsan-ri, Hwangseoksan Mt.), 151023-054[HNHM], Geomangsan Mt., 180731-037 [HNHM]

254. Lespedeza maximowiczii C. K. Schneid. 조록싸리O, P[1, 2, 3, 4] Geochang-gun (Sangcheon-ri, Geumwonsan Mt.), 110604101 [HNHM], Hamyang-gun (Sangwon-ri, Gibaeksan Mt.), 150605-022 [KB]; Hwangseoksan Mt., 160922-007[KB]; Geomangsan Mt., 180619-046 [KB]

$\star 255$. Lespedeza thunbergii Nakai subsp. formosa(Vogel) H. Ohashi 풀싸리 $\mathrm{O}, \mathrm{P}[3]$

Hwangseoksan Mt., 160922-014 [KB]

256. Maackia amurensis Rupr.다릅나무 T $[2,3]$

Geochang-gun (Gohak-ri, Gibaeksan Mt.), 150709-015[KB]; Hamyang-gun (Hwangsan-ri, Hwangseoksan Mt.),150604-035 [HNHM]

257. Pueraria lobata (Willd.) Ohwi 칡 E, M, P, S [1, 3, 4] Geochang-gun (Sangcheon-ri, Geumwonsan Mt.), 110830178 [HNHM]; Hamyang-gun (Sangwon-ri, Hwangseoksan Mt.), 150813-133 [HNHM]; Geomangsan Mt., 180731-041[KB]

258. Rhynchosia acuminatifolia Makino 큰여우콩 $\mathrm{P}[2]$ Geochang-gun (Gohak-ri, Gibaeksan Mt.), 150812-058[KB]

259. Sophora flavescens Solander ex Aiton 고삼 M [2, 3] Geochang-gun (Gohak-ri, Gibaeksan Mt.), 150709-078[KB]; Hamyang-gun (Hwangsan-ri, Hwangseoksan Mt.),150604-001 [HNHM]

^260. Styphnolobium japonicum (L.) Schott 회화나무(식)M[2] Geochang-gun (Gohak-ri, Gibaeksan Mt.), 150812-187[KB]

261. Trifolium repens $\mathrm{L}$. 토끼풀 $\mathrm{P}$ [1]

Geochang-gun (Sangcheon-ri, Geumwonsan Mt.), 110604054 [HNHM]

262. Vicia amoena Fisch. ex Ser. 갈퀴나물 $\mathrm{E}, \mathrm{P}[2]$ Geochang-gun (Gohak-ri, Gibaeksan Mt.), 150709-043[KB]

$\star 263$. Vicia hirticalycina Nakai 나래완두 $\mathrm{P}[1]$ Geochang-gun (Sangcheon-ri, Geumwonsan Mt.), 110518001 [HNHM]

264. Vicia unijuga A. Braun나비나물 E, $\mathrm{P}[2,3]$ Geochang-gun (Gohak-ri, Gibaeksan Mt.), 150709-081[KB]; Hamyanggun (Bongjeon-ri, Hwangseoksan Mt.),151022-015 [HNHM]
265. Vicia venosa (Willd.) Maxim. 연리갈퀴 $\mathrm{P}[4]$ Geomangsan Mt.,180731-361 [KB]

$\star 266$. Vigna angularis (Willd.) Ohwi \& H. Ohashi var. nipponensis(Ohwi) Ohwi \& H. Ohashi 새팥E[3] Hamyang-gun (Sangwon-ri, Hwangseoksan Mt.), 150813$029[\mathrm{HNHM}]$

\section{Oxalidaceae 갱이밥과}

267. Oxalis corniculata L. 괭이밥 E, M [4] Geomangsan Mt.,180619-235 [KB]

268. Oxalis obtriangulata Maxim. 큰괭이밥E[3, 4] Hamyang-gun (Sangwon-ri, Hwangseoksan Mt.), 150423016[HNHM]; Geomangsan Mt., 180419-011 [KB]

$\star 269$. Oxalis stricta L.선괭이밥 E [3] Hamyang-gun (Hwangsan-ri, Hwangseoksan Mt.), 150604 $178[\mathrm{HNHM}]$

\section{Geraniaceae 쥐손이풀과}

270. Geranium krameri Franch. \& Sav. 선이질풀 M[3] Hwangseoksan Mt., 160921-134 [KB]

271. Geranium sibiricum L.쥐손이풀 M, P [2, 3] Hamyang-gun (Sangwon-ri, Gibaeksan Mt.), 150917-032[KB]; Hwangseoksan Mt., 160921-005 [HNHM]

272. Geranium thunbergii Siebold ex Lindl. \& Paxton 이질풀 M, $\mathrm{P}[1,2,3,4]$

Geochang-gun (Sangcheon-ri, Geumwonsan Mt.), 110830212[HNHM]; Geochang-gun (Gohak-ri, Gibaeksan Mt.),150709-076 [KB]; Hamyang-gun (Bongjeon-ri, Hwangseoksan Mt.), 151022-064[HNHM]; Geomangsan Mt., 180731-095 [KB]

273. Geranium wilfordii Maxim. 세잎쥐손이 M[1] Geochang-gun (Sangcheon-ri, Geumwonsan Mt.), 110830270[HNHM]

\section{Euphorbiaceae 대극과}

274. Acalypha australis L.깨풀E[3]

Hamyang-gun (Sangwon-ri, Hwangseoksan Mt.), 150813043 [HNHM]

275. Euphorbia sieboldiana Morren \& Decne. 개감수 M[3, 4] Geochang-gun (Sangcheon-ri, Geumwonsan Mt.), 110604$070[\mathrm{HNHM}]$; Geochang-gun (Gohak-ri, Gibaeksan Mt.),150812-108 [KB]; Hamyang-gun (Bongjeon-ri, Hwangseoksan Mt.), 150710-006[HNHM]; Geomangsan Mt., 180619-114 [KB]

276. Neoshirakia japonica (Siebold \& Zucc.) Esser 사람주나무 $\mathrm{S}[2,3,4]$

Hamyang-gun (Sangwon-ri, Gibaeksan Mt.), 150605-134[KB]; Hamyang-gun (Sangwon-ri, Hwangseoksan Mt.),150813-129 [HNHM]; Geomangsan Mt., 180619-035[KB]

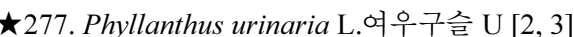

Geochang-gun (Gohak-ri, Gibaeksan Mt.), 150812-035[KB]; Hwangseoksan Mt., 180801-081 [KB]

278. Securinega suffruticosa (Pall.) Rehder 광대싸리 M[1, 2, 3] Hamyang-gun (Sangwon-ri, Geumwonsan Mt.), 110716119 [HNHM]; Geochang-gun (Gohak-ri, Gibaeksan Mt.), 150709-004 [KB]; Hamyang-gun (Hwangsan-ri, Hwangseoksan Mt.), 150604-065[HNHM]

Rutaceae 운향과

279. Phellodendron amurense Rupr. 황벽나무 M, P[3] Hwangseoksan Mt., 180619-046 [KB]

280. Poncirus trifoliata Raf. 탱자나무(식)M, O[1] Geochang-gun (Wolseong-ri, Geumwonsan Mt.), 110514042[HNHM] 
Appendix 1. Continued.

$\star$ 281. Tetradium daniellii (Benn.) T. G. Hartley 쉬나무 (식)M[2] Geochang-gun (Gohak-ri, Gibaeksan Mt.), 150709-134[KB]

282. Zanthoxylum piperitum (L.) DC. 초피나무E, $\mathrm{M}[1,2,3]$ Geochang-gun (Changseon-ri, Geumwonsan Mt.), 110519 031 [HNHM], Hamyang-gun (Sangwon-ri, Gibaeksan Mt.), 150605-005 [KB]; Hamyang-gun (Sangwon-ri, Hwangseoksan Mt.), 150813-053[HNHM]

283. Zanthoxylum schinifolium Siebold \& Zucc. 산초나무E[1, 2, 3, 4] Geochang-gun (Sangcheon-ri, Geumwonsan Mt.), 110830262[HNHM]; Geochang-gun (Gohak-ri, Gibaeksan Mt.),150709-089 [KB]; Hamyang-gun (Sangwon-ri, Hwangseoksan Mt.), 150813117[HNHM]; Geomangsan Mt., 180731-158 [KB]

Polygalaceae 원지과

284. Polygala japonica Houtt. 애기풀 E, M[1, 2] Geochang-gun (Sangcheon-ri, Geumwonsan Mt.), 110518007[HNHM]; Geochang-gun (Sangcheon-ri, Gibaeksan Mt.), 151015-023 [KB]

Anacardiaceae 옻나무과

285. Rhus chinensis Mill.붉나무 E, M, R [2, 3]

Geochang-gun (Gohak-ri, Gibaeksan Mt.), 150812-219[KB]; Hamyang-gun (Sangwon-ri, Hwangseoksan Mt.),150813-122 [HNHM]

286. Toxicodendron trichocarpum(Miq.) Kuntze 개옻나무 $\mathrm{M}[1,2,3,4]$ Geochang-gun (Sangcheon-ri, Geumwonsan Mt.), 110515007[HNHM]; Hamyang-gun (Sangwon-ri, Gibaeksan Mt.), 150605-162 [KB]; Hamyang-gun (Hwangsan-ri, Hwangseoksan Mt.), 150604-191[HNHM]; Geomangsan Mt., 180619-082 [KB]

$\star 287$. Toxicodendron vernicifluum(Stokes) F. A. Barkley 옻나무( 식)M, R[3]

Hamyang-gun (BongJeon-ri, Hwangseoksan Mt.), 150519 013 [HNHM]

\section{Aceraceae 단풍나무과}

288. Acer buergerianum Miq. 중국단풍 O [3]

Hamyang-gun (Bongjeon-ri, Hwangseoksan Mt.), 151022109 [HNHM]

289. Acer komarovii Pojark.시닥나무 O [2]

Hamyang-gun (Sangwon-ri, Gibaeksan Mt.), 150917-045[KB]

290. Acer palmatum Thunb.단풍나무 O, P [1, 3]

Geochang-gun (Sangcheon-ri, Geumwonsan Mt.), 110518-

166[HNHM]; Hamyang-gun (Bongjeon-ri, Hwangseoksan Mt.), 151022-115 [KB]

$\star 291$. Acer pictum Thunb.털고로쇠나무 M, O, P [1] Geochang-gun (Changseon-ri, Geumwonsan Mt.), 110519 033[HNHM]

292. Acer pictum Thunb. subsp. mono(Maxim.) Ohashi 고로쇠나무 $\mathrm{M}, \mathrm{O}, \mathrm{P}[2,3,4]$

Hamyang-gun (Sangwon-ri, Gibaeksan Mt.), 150424-064[KB];

Hamyang-gun (Sangwon-ri, Hwangseoksan Mt.),150423-007

[HNHM], Geomangsan Mt., 180418-025[KB]

293. Acer pseudosieboldianum (Pax) Kom. 당단풍나무(식)O[1, 2, 3, 4] Geochang-gun (Wolseong-ri, Geumwonsan Mt.), 110514025[HNHM]; Hamyang-gun (Sangwon-ri, Gibaeksan Mt.), 150424-070 [KB]; Hamyang-gun (Bongjeon-ri, Hwangseoksan Mt.), 150710-008[HNHM]; Geomangsan Mt., 180619-232 [KB]

294. Acer tataricum L. subsp. ginnala(Maxim.) Wesm. 신나무 P[1, 2] Geochang-gun (Changseon-ri, Geumwonsan Mt.), 110519025[HNHM]; Geochang-gun (Gohak-ri, Gibaeksan Mt.), $150709-149$ [KB]

Balsaminaceae 봉선화과

295. Impatiens noli-tangere $\mathrm{L}$. 노랑물봉선 $\mathrm{P}[1]$
Geochang-gun (Sangcheon-ri, Geumwonsan Mt.), 110830074 [HNHM]

296. Impatiens textori Miq. 물봉선 M [1, 2, 3] Geochang-gun (Sangcheon-ri, Geumwonsan Mt.), 110830169 [HNHM]; Hamyang-gun (Sangwon-ri, Gibaeksan Mt.), 150917-146 [KB]; Hwangseoksan Mt., 160921-115[KB]

\section{Aquifoliaceae 감탕나무과}

297. Ilex macropoda Miq. 대팻집나무 $\mathrm{E}[1,2,3]$

Geochang-gun (Sangcheon-ri, Geumwonsan Mt.), 110604044[HNHM]; Geochang-gun (Gohak-ri, Gibaeksan Mt.), 150812-214 [KB]; Hwangseoksan Mt., 170525-187[KB]

$\star 298$. Ilex serrata Thunb. 낙상홍 (식) O [1]

Geochang-gun (Wolseong-ri, Geumwonsan Mt.), 110918074[HNHM]

\section{Celastraceae 노박덩굴과}

299. Celastrus orbiculatus Thunb. 노박덩굴E, O, R[1, 2] Geochang-gun (Changseon-ri, Geumwonsan Mt.), 110519150[HNHM]; Geochang-gun (Gohak-ri, Gibaeksan Mt.), 150709-003 [KB]

300. Euonymus alatus (Thunb.) Siebold 화살나무E, M, P[1, 3] Geochang-gun (Sangcheon-ri, Geumwonsan Mt.), 110518018 [HNHM]; Hamyang-gun (Hwangsan-ri, Hwangseoksan Mt.), 151023-049 [HNHM]

301. Euonymus alatus (Thunb.) Siebold f. ciliatodentatus(Franch. \& Sav.) Hiyama 회잎나무 E, P [2, 3, 4] Hamyang-gun (Sangwon-ri, Gibaeksan Mt.), 151016-074[KB]; Hamyang-gun (Sangwon-ri, Hwangseoksan Mt.), 150423-050 [HNHM]; Geomangsan Mt., 180619-125[KB]

302. Euonymus hamiltonianus Wall. 참빗살나무 $\mathrm{M}, \mathrm{P}[2]$ Hamyang-gun (Sangwon-ri, Gibaeksan Mt.), 151016-072[KB]

303. Euonymus oxyphyllus Miq. 참회나무 $\mathrm{P}[1,2,3,4]$ Geochang-gun (Sangcheon-ri, Geumwonsan Mt.), 110518$020[\mathrm{HNHM}]$; Hamyang-gun (Sangwon-ri, Gibaeksan Mt.),150605-049 [KB]; Hamyang-gun (Bongjeon-ri, Hwangseoksan Mt.), 150710-017[HNHM]; Geomangsan Mt., 180619-040 [KB]

304. Euonymus sachalinensis (F. Schmidt) Maxim. 회나무 U[1] Geochang-gun (Sangcheon-ri, Geumwonsan Mt.), 110830101 [HNHM]

305. Tripterygium regelii Sprague \& Takeda 미역줄나무U[1, 2, 3, 4] Geochang-gun (Gohak-ri, Gibaeksan Mt.), 150812-019[KB]

Staphyleaceae 고추나무과

306. Staphylea bumalda DC.고추나무 E [1, 2, 3, 4] Geochang-gun (Wolseong-ri, Geumwonsan Mt.), 110514077[HNHM]; Hamyang-gun (Sangwon-ri, Gibaeksan Mt.), 150605-072 [KB]; Hamyang-gun (Bongjeon-ri, Hwangseoksan Mt.), 150710-073[HNHM]; Geomangsan Mt., 180619-007 [KB]

Rhamnaceae 갈매나무과

$\star$ 307. Hovenia dulcis Thunb.헛개나무 (식) E [2, 3] Geochang-gun (Gohak-ri, Gibaeksan Mt.), 150709-111[KB]; Hwangseoksan Mt., 170713-076 [KB]

308. Rhamnus yoshinoi Makino짝자래나무 $\mathrm{U}[1,3]$

Geochang-gun (Changseon-ri, Geumwonsan Mt.), 110519117 [HNHM], Hamyang-gun (Hwangsan-ri, Hwangseoksan Mt.), 150604-196 [HNHM]

Vitaceae 포도과

309. Ampelopsis heterophylla (Thunb.) Siebold \& Zucc. 개머루 $\mathrm{U}[1,2,3,4]$ 
Appendix 1. Continued.

\begin{tabular}{|c|c|}
\hline $\begin{array}{l}\text { Hamyang-gun (Sangwon-ri, Geumwonsan Mt.), 110716- } \\
\text { 108[HNHM]; Geochang-gun (Gohak-ri, Gibaeksan } \\
\text { Mt.),150709-091 [KB]; Hamyang-gun (Bongjeon-ri, } \\
\text { Hwangseoksan Mt.), 151022-071[HNHM]; Geomangsan Mt., } \\
\text { 180731-218 [KB] }\end{array}$ & $\begin{array}{l}\text { Geochang-gun (Wolseong-ri, Geumwonsan Mt.), 110514- } \\
081 \text { [HNHM]; Hamyang-gun (Hwangsan-ri, Hwangseoksan } \\
\text { Mt.),150423-004 [HNHM]; Geomangsan Mt., 180418- } \\
\text { 110[HNHM] } \\
\text { 324. Viola orientalis (Maxim.) W. Becker 노랑제비꽃 U[1, 3, 4] }\end{array}$ \\
\hline $\begin{array}{l}\text { 310. Parthenocissus tricuspidata(Siebold \& Zucc.) Planch. 담쟁이 } \\
\text { 덩굴 O, P }[1,3] \\
\text { Geochang-gun (Sangcheon-ri, Geumwonsan Mt.), 110830- }\end{array}$ & $\begin{array}{l}\text { Geochang-gun (Sangcheon-ri, Geumwonsan Mt.), 110420- } \\
\text { 038[HNHM]; Hwangseoksan Mt., 170525-243 [KB]; } \\
\text { Geomangsan Mt.,180419-041 [KB] }\end{array}$ \\
\hline $\begin{array}{l}\text { 054[HNHM]; Hamyang-gun (Hwangsan-ri, Hwangseoksan Mt.), } \\
\text { 150604-015 [HNHM] } \\
\text { 311. Vitis amurensis Rupr.왕머루 E, M, P [3] }\end{array}$ & $\begin{array}{l}\text { 325. Viola patrinii DC. ex Ging. 흰제비꽃 U[1] } \\
\text { Geochang-gun (Sangcheon-ri, Geumwonsan Mt.), 110515- } \\
\text { 013[HNHM] }\end{array}$ \\
\hline $\begin{array}{l}\text { 11. Vitis amurensis Rupr.왕머루 E, M, P [3] } \\
\text { Hamyang-gun (Bongjeon-ri, Hwangseoksan Mt.), 150519- } \\
014 \text { [HNHM] }\end{array}$ & $\begin{array}{l}\text { 326. Viola philippica Cav.호제비꽃 U [2, 3] } \\
\text { Hamyang-gun (Sangwon-ri, Gibaeksan Mt.), 150424-027[KB]; }\end{array}$ \\
\hline $\begin{array}{l}\text { 312. Vitis flexuosa Thunb.새머루 E, P }[1,3] \\
\text { Geochang-gun (Sangcheon-ri, Geumwonsan Mt.), 110518- }\end{array}$ & $\begin{array}{l}\text { Hamyang-gun (Sangwon-ri, Hwangseoksan Mt.),150423-004 } \\
\text { [HNHM] }\end{array}$ \\
\hline $\begin{array}{l}061 \text { [HNHM]; Hamyang-gun (Hwangsan-ri, Hwangseoksan } \\
\text { Mt.),150604-029 [HNHM] }\end{array}$ & $\begin{array}{l}\text { 327. Viola rossii Hemsl.고깔제비꽃 M }[1,2,3,4] \\
\text { Geochang-gun (Sangcheon-ri, Geumwonsan Mt.), 110519- }\end{array}$ \\
\hline Tiliaceae 피나무과 & 024[HNHM]; Hamyang-gun (Sangwon-ri, Gibaeksan \\
\hline $\begin{array}{l}\text { 313. Corchoropsis tomentosa (Thunb.) Makino 수까치깨U[1, 3] } \\
\text { Geochang-gun (Sangcheon-ri, Geumwonsan Mt.), 110830- } \\
\text { 255[HNHM]; Hamyang-gun (Sangwon-ri, Hwangseoksan Mt.), } \\
150813-109 \text { [HNHM] }\end{array}$ & $\begin{array}{l}\text { Mt.),150424-014 [KB]; Hamyang-gun (Sangwon-ri, } \\
\text { Hwangseoksan Mt.), 150423-015[HNHM], Geomangsan Mt., } \\
\text { 180419-056 [KB] } \\
\text { 328. Viola selkirkii Pursh ex Goldie 뫼제비꽃U[3] }\end{array}$ \\
\hline 314. Tilia taquetii C. K. Schneid. 뽕잎피나무P, T[2, 4] & \\
\hline $\begin{array}{l}\text { Hamyang-gun (Sangwon-ri, Gibaeksan Mt.), } 150917-172[\mathrm{~KB}] \\
\text { Geomangsan Mt., } 180619-021 \text { [KB] }\end{array}$ & $\begin{array}{l}\text { 329. Viola variegata Fisch. ex Link 알록제비꽃 E, } \mathrm{O}[2,3,4] \\
\text { Hamyang-gun (Sangwon-ri, Gibaeksan Mt.), } 151016-052[\mathrm{~KB}]\end{array}$ \\
\hline $\begin{array}{l}\text { Elaeagnaceae 보리수나무과 } \\
\text { 315. Elaeagnus umbellata Thunb. 보리수나무E, } \mathrm{P}[1,2,3]\end{array}$ & $\begin{array}{l}\text { Hamyang-gun (Hwangsan-ri, Hwangseoksan Mt.),150604-003 } \\
\text { [HNHM], Geomangsan Mt., 180418-010[KB] }\end{array}$ \\
\hline Geoch & Cucurbitaceae 박과 \\
\hline $\begin{array}{l}040[\mathrm{HNHM}] \text {; Hamyang-gun (Sangwon-ri, Gibaeksan } \\
\text { Mt.),150605-156 [KB]; Hamyang-gun (Bongjeon-ri, } \\
\text { Hwangseoksan Mt.), 150519-027[HNHM] }\end{array}$ & $\begin{array}{l}\text { 330. Trichosanthes kirilowii Maxim. 하늘타리E, M[2] } \\
\text { Geochang-gun (Gohak-ri, Gibaeksan Mt.), 150812-174[KB] } \\
\text { Onagraceae 바늘꽃과 }\end{array}$ \\
\hline Violaceae 제비꽃과 & $\begin{array}{l}\text { alpina } \mathrm{L} \text {. 쥐털이슬 U [4] } \\
\text { Mt.,180731-063 [KB] }\end{array}$ \\
\hline $\begin{array}{l}\text { 방제비닟 E }[1,2,3,4] \\
\text { Geumwonsan Mt.), } 110514 \text { - } \\
\text { (Sangwon-ri, Gibaeksan }\end{array}$ & $\begin{array}{l}\text { Geon } \\
\text { 332. } C i \\
\text { Geon }\end{array}$ \\
\hline $\begin{array}{l}\text { Mt.), 150605-115 [KB]; Hamyang-gun (Hwangsan-ri, } \\
\text { Hwangseoksan Mt.), 150423-001[HNHM]; Geomangsan Mt., } \\
\text { 180619-054 [KB] }\end{array}$ & $\begin{array}{l}\text { 333. Circaea lutetiana L. subsp. quadrisulcata(Maxim.) Asch. \& } \\
\text { Magnus 말털이슬 P [3, 4] } \\
\text { Hwangseoksan Mt.,180801-116 [HNHM],; Geomangsan Mt., }\end{array}$ \\
\hline 317. Viola albida Palib.태백제비꽃 E [1, 2, 3, 4] & \\
\hline $\begin{array}{l}\text { Geochang-gun (Sangcheon-ri, Geumwonsan Mt.), 110420- } \\
\text { 014[HNHM]; Hamyang-gun (Sangwon-ri, Gibaeksan Mt.), } \\
\text { 150918-118 [KB]; Hamyang-gun (Sangwon-ri, Hwangseoksan } \\
\text { Mt.), 150423-017[HNHM]; Geomangsan Mt., 180418-065 [KB] }\end{array}$ & $\begin{array}{l}\star \text { 334. Circaea mollis Slebold \& Zucc. 털이슬 P[2] } \\
\text { Gibaeksan Mt.),150709-047 [KB]; Hamyang-gun (Sangwon-ri, } \\
\text { Hwangseoksan Mt.), 150423-001[HNHM]; Geomangsan Mt., } \\
\text { 180419-060 [KB] }\end{array}$ \\
\hline $\begin{array}{l}\text { ^318. Viola albida Palib. var. takahashii(Makino) Nakai 단풍제비 } \\
\text { 꽃U[3] } \\
\text { Hamyang-gun (Hwangsan-ri, Hwangseoksan Mt.), 150604- } \\
\text { 199[HNHM] }\end{array}$ & $\begin{array}{l}\text { 335. Oenothera biennis L. 달맞이꽃 O, P }[1,2,4] \\
\text { Geochang-gun (Sangcheon-ri, Geumwonsan Mt.), 110830- } \\
\text { 064[HNHM]; Hamyang-gun (Sangwon-ri, Gibaeksan Mt.), } \\
\text { 150917-202 [KB]; Geomangsan Mt., 180731-107[KB] }\end{array}$ \\
\hline $\begin{array}{l}\text { 319. Viola arcuata Blume콩제비꽃 E, P [1, 3] } \\
\text { Geochang-gun (Sangcheon-ri, Geumwonsan Mt.), 110420- } \\
\text { 017[HNHM]; Hamyang-gun (Hwangsan-ri, Hwangseoksan } \\
\text { Mt.),150604-172 [HNHM] }\end{array}$ & $\begin{array}{l}\text { Halorragaceae 개미탑과 } \\
\text { ^336. Haloragis micrantha (Thunb.) R.Br. ex Siebold \& Zucc. 개 } \\
\text { 미탑 U[2] } \\
\text { Geochang-gun (Gohak-ri, Gibaeksan Mt.), 150812-036[KB] }\end{array}$ \\
\hline $\begin{array}{l}\text { 320. Viola japonica Langsd. ex Ging. 왜제비꽃 U[3] } \\
\text { Hamyang-gun (Sangwon-ri, Hwangseoksan Mt.), 150423- } \\
\text { 034[HNHM] }\end{array}$ & $\begin{array}{l}\text { Alangiaceae 박쥐나무과 } \\
\text { 337. Alangium platanifolium (Siebold \& Zucc.) Harms var. } \\
\text { trilobum(Miq.) Ohwi 박쥐나무E, P[1, 2, 3, 4] }\end{array}$ \\
\hline $\begin{array}{l}\text { 321. Viola keiskei Miq.잔털제비꽃 U [3] } \\
\text { Hamyang-gun (Sangwon-ri, Hwangseoksan Mt.), 150423- } \\
\text { 033[HNHM] }\end{array}$ & $\begin{array}{l}\text { Hamyang-gun (Sangwon-ri, Geumwonsan Mt.), 110716- } \\
096[\mathrm{HNHM}] \text {; Hamyang-gun (Sangwon-ri, Gibaeksan } \\
\text { Mt.),150605-040 [KB]; Hamyang-gun (Bongjeon-ri, }\end{array}$ \\
\hline $\begin{array}{l}\text { 322. Viola lactiflora Nakai흰젓제비꽃 U [2] } \\
\text { Hamyang-gun (Sangwon-ri, Gibaksan Mt.), 150424-029 }\end{array}$ & $\begin{array}{l}\text { Hwangseoksan Mt.), 150710-022[HNHM]; Geomangsan Mt., } \\
\text { 180619-128 [KB] }\end{array}$ \\
\hline 323. Viola mandshurica $\mathrm{W}$. Becker 제비꽃 $\mathrm{E}, \mathrm{M}, \mathrm{P}[1,3,4]$ & Cornaceae 충충나무과 \\
\hline
\end{tabular}


Appendix 1. Continued.

338. Cornus controversa Hemsl. 층층나무 $\mathrm{U}[1,2,3,4]$ Geochang-gun (Wolseong-ri, Geumwonsan Mt.), 110514043[HNHM]; Hamyang-gun (Sangwon-ri, Gibaeksan Mt.),150605-105 [KB]; Hamyang-gun (Bongjeon-ri, Hwangseoksan Mt.), 150519-015[HNHM]; Geomangsan Mt., 180731-073 [KB]

339. Cornus kousa F.Buerger ex Miquel 산딸나무U[1, 4] Geochang-gun (Changseon-ri, Geumwonsan Mt.), 110519 081 [HNHM]; Geomangsan Mt., 180731-260 [KB]

340. Cornus officinalis Siebold \& Zucc. 산수유 (식)M, O[2, 3] Hamyang-gun (Sangwon-ri, Gibaeksan Mt.), 150605-187[KB]; Hwangseoksan Mt., 160922-034 [KB]

341. Cornus walteri Wangerin말채나무 U [1] Hamyang-gun (Sangwon-ri, Geumwonsan Mt.), 110716114 [HNHM]

\section{Araliaceae 두릅나무과}

342. Aralia cordata Thunb. var. continentalis(Kitag.) Y. C. Chu 독 활 $\mathrm{E}, \mathrm{M}$ [1]

Geochang-gun (Wolseong-ri, Geumwonsan Mt.), 110918013 [HNHM]

343. Aralia elata (Miq.) Seem. 두릅나무E, M[2] Geochang-gun (Gohak-ri, Gibaeksan Mt.), 150812-207[KB]

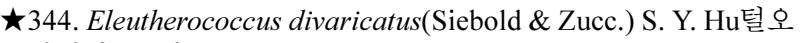
갈피나무 (식) $\mathrm{M}$ [3]

Hamyang-gun (Hwangsan-ri, Hwangseoksan Mt.), 150604 $190[\mathrm{HNHM}]$

345. Eleutherococcus sessiliflorus(Rupr. \& Maxim.) S. Y. Hu오갈 피나무 (식) E, M [3]

Hamyang-gun (Sangwon-ri, Hwangseoksan Mt.), 150813046[HNHM]

\section{Apiaceae 산형과}

346. Angelica cartilaginomarginata(Makino ex Y. Yabe) Nakai처 녀바디 U [3]

Hwangseoksan Mt., 160921-117 [KB]

347. Angelica decursiva (Miq.) Franch. \& Sav. 바디나물E, M[1, 2, 3] Geochang-gun (Sangcheon-ri, Geumwonsan Mt.), 110830040 [HNHM], Hamyang-gun (Sangwon-ri, Gibaeksan Mt.), 150918-198 [KB]; Hamyang-gun (Bongjeon-ri, Hwangseoksan Mt.), 151022-012[HNHM]

348. Angelica polymorpha Maxim. 궁궁이 E, M[1, 2, 3] Geochang-gun (Sangcheon-ri, Geumwonsan Mt.), 110830192 [HNHM]; Hamyang-gun (Sangwon-ri, Gibaeksan Mt.), 150917-268 [KB]; Hamyang-gun (Bongjeon-ri, Hwangseoksan Mt.), 151022-042[HNHM]

349. Angelica reflexa B.Y. Lee 강활 E, M [1, 2, 3] Geochang-gun (Sangcheon-ri, Geumwonsan Mt.), 110830026[HNHM]; Hamyang-gun (Sangwon-ri, Gibaeksan Mt.), 150918-036 [HNHM]; Hwangseoksan Mt., 160922-065[KB]

$\star$ 350. Anthriscus sylvestris (L.) Hoffm. 전호 M, P [3, 4] Hwangseoksan Mt., 170525-228 [KB]; Geomangsan Mt., 180619-184[KB]

351. Cryptotaenia japonica Hassk. 파드득나물 $\mathrm{E}[3]$ Hwangseoksan Mt., 170713-033 [KB]

352. Cymopterus melanotilingia (H. Boissieu) C.Y.Yoon 큰참나물 $\mathrm{E}[2,3]$

Hamyang-gun (Sangwon-ri, Gibaeksan Mt.), 150917-048[KB]; Hwangseoksan Mt., 160921-116 [KB]

353. Heracleum moellendorffii Hance 어수리 E, P[3] Hamyang-gun (Bongjeon-ri, Hwangseoksan Mt.), 151022$013[\mathrm{HNHM}]$
354. Oenanthe javanica (Blume) DC. 미나리 E, M[2] Geochang-gun (Gohak-ri, Gibaeksan Mt.), 150812-022[KB]

355. Osmorhiza aristata (Thunb.) Rydb. 긴사상자U[4] Geomangsan Mt.,180619-016 [KB]

356. Ostericum grosseserratum (Maxim.) Kitag. 신감채 $\mathrm{P}[2,3]$ Geochang-gun (Gohak-ri, Gibaeksan Mt.), 150812-127[KB]; Hamyang-gun (Sangwon-ri, Hwangseoksan Mt.),150813-011 [HNHM]

357. Peucedanum terebinthaceum (Fisch. ex Trevir.) Fisch. ex Turcz. 기름나물 $\mathrm{E}[1,2,3]$

Geochang-gun (Sangcheon-ri, Geumwonsan Mt.), 110830057[HNHM]; Geochang-gun (Sangcheon-ri, Gibaeksan Mt.), 151015-005 [KB]; Hamyang-gun (Hwangsan-ri, Hwangseoksan Mt.), 151023-062[HNHM]

358. Pimpinella brachycarpa (Kom.) Nakai 참나물 E, $\mathrm{P}[1,3]$ Geochang-gun (Sangcheon-ri, Geumwonsan Mt.), 110830117 [HNHM]; Hamyang-gun (Bongjeon-ri, Hwangseoksan Mt.), 151022-014 [HNHM]

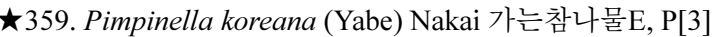
Hamyang-gun (Bongjeon-ri, Hwangseoksan Mt.), 151022$061[\mathrm{HNHM}]$

360. Sanicula chinensis Bunge 참반디 E, M[1, 3, 4] Hamyang-gun (Sangwon-ri, Geumwonsan Mt.), 110716001 [HNHM]; Hamyang-gun (Bongjeon-ri, Hwangseoksan Mt.),150710-033 [HNHM]; Geomangsan Mt., 180731-272[KB]

^361. Sium ninsi L.감자개발나물 U [3] Hamyang-gun (Bongjeon-ri, Hwangseoksan Mt.), 151022124[HNHM]

362. Sium suave Walter개발나물 $M[1,2]$ Geochang-gun (Sangcheon-ri, Geumwonsan Mt.), 110830$160[\mathrm{HNHM}]$; Hamyang-gun (Sangwon-ri, Gibaeksan Mt.), 150918-081 [KB]

363. Torilis japonica (Houtt.) DC. 사상자 $\mathrm{M}[1,2]$ Hamyang-gun (Sangwon-ri, Geumwonsan Mt.), 110716107[HNHM]; Geochang-gun (Gohak-ri, Gibaeksan Mt.), 150709-045 [KB]

\section{Ericaceae 진달래과}

364. Chimaphila japonica Miq. 매화노루발U[2, 3] Geochang-gun (Gohak-ri, Gibaeksan Mt.), 150709-025[KB]; Hwangseoksan Mt., 170713-100 [KB]

365. Pyrola japonica Klenze ex Alef. 노루발 M[1, 2, 3, 4] Geochang-gun (Changseon-ri, Geumwonsan Mt.), 110519 005 [HNHM], Hamyang-gun (Sangwon-ri, Gibaeksan Mt.), 150917-050 [KB]; Hamyang-gun (Hwangsan-ri, Hwangseoksan Mt.), 150604-062[HNHM], Geomangsan Mt., 180619-049 [KB]

366. Rhododendron mucronulatum Turcz. 진달래 E, $\mathrm{O}[1,2,3,4]$ Geochang-gun (Sangcheon-ri, Geumwonsan Mt.), 110420008 [HNHM]; Geochang-gun (Gohak-ri, Hamyang-gun (Sangwon-ri, Geumwonsan Mt.), 110716-098[HNHM]; Hamyang-gun (Hwangsan-ri, Hwangseoksan Mt.),150604-080 [KB]

367. Rhododendron schlippenbachiiMaxim. 철쭉 $\mathrm{O}[1,2,3,4]$ Geochang-gun (Sangcheon-ri, Geumwonsan Mt.), 110514025[HNHM]; Hamyang-gun (Sangwon-ri, Gibaeksan Mt.),150917-112 [KB]; Hamyang-gun (Hwangsan-ri, Hwangseoksan Mt.), 150423-016[HNHM]; Geomangsan Mt., 180619-018 [KB]

368. Rhododendron yedoense Maxim. f. poukhanense(H.Lév.) M. Sugim. ex T. Yamaz.산철쭉 O $[1,2,3,4]$ 
Appendix 1. Continued.

\begin{tabular}{l}
\hline \hline Geochang-gun (Sangcheon-ri, Geumwonsan Mt.), 110514- \\
021 [HNHM]; Hamyang-gun (Sangwon-ri, Gibaeksan \\
Mt.),150424-004 [KB]; Hamyang-gun (Hwangsan-ri, \\
Hwangseoksan Mt.), 150423-014[HNHM], Geomangsan Mt., \\
180418-038 [KB]
\end{tabular}

369. Vaccinium hirtum Thunb. var. koreanum(Nakai) Kitam. 산앵 도나무 $\mathrm{E}[1,3]$

Geochang-gun (Sangcheon-ri, Geumwonsan Mt.), 110830243[HNHM]; Hwangseoksan Mt., 170525-063 [KB]

370. Vaccinium oldhamii Miq. 정금나무 $\mathrm{E}[2,3]$

Hamyang-gun (Sangwon-ri, Gibaeksan Mt.), 150918-149[KB]; Hamyang-gun (Bongjeon-ri, Hwangseoksan Mt.), 150710-018 [HNHM]

\section{Primulaceae 앵초과}

^371. Androsace umbellata (Lour.) Merr. 봄맞이 U[3] Hamyang-gun (Hwangsan-ri, Hwangseoksan Mt.), 150423003[HNHM]

372. Lysimachia clethroides Duby 큰까치수염 $\mathrm{E}, \mathrm{P}[1,2,3,4]$ Geochang-gun (Changseon-ri, Geumwonsan Mt.), 110519021 [HNHM]; Geochang-gun (Gohak-ri, Gibaeksan Mt.),150709-164 [KB]; Hamyang-gun (Bongjeon-ri, Hwangseoksan Mt.), 150710-061[HNHM]; Geomangsan Mt., 180731-340 [KB]

$\star$ 373. Lysimachia japonica Thunb. 좀가지풀U[1, 3, 4] Geochang-gun (Sangcheon-ri, Geumwonsan Mt.), 110830257[HNHM]; Hamyang-gun (Hwangsan-ri, Hwangseoksan Mt.),150604-075 [HNHM]; Geomangsan Mt., 180619-069[KB]

374. Lysimachia vulgaris L. var. davurica(Ledeb.) R. Kunth 좁쌀풀 $\mathrm{U}[2]$

Geochang-gun (Gohak-ri, Gibaeksan Mt.), 150709-063[KB]

Ebenaceae 감나무과

375. Diospyros lotus L.고욤나무 E, M [2, 3]

Hamyang-gun (Sangwon-ri, Gibaeksan Mt.), 150605-169[KB]; Hamyang-gun (Hwangsan-ri, Hwangseoksan Mt.),150604-076 [HNHM]

\section{Styracaceae 때죽나무과}

376. Styrax japonicus Siebold \& Zucc. 때죽나무O, P[1, 2, 3$]$ Hamyang-gun (Sangwon-ri, Geumwonsan Mt.), 110716104[HNHM]; Hamyang-gun (Sangwon-ri, Gibaeksan Mt.),150605-044 [KB]; Hamyang-gun (Bongjeon-ri, Hwangseoksan Mt.), 150519-001[HNHM]

377. Styrax obassia Siebold \& Zucc. 쪽동백나무O, P[1, 3, 4] Geochang-gun (Sangcheon-ri, Geumwonsan Mt.), 110518103[HNHM]; Hamyang-gun (Bongjeon-ri, Hwangseoksan Mt.),150519-020 [HNHM]; Geomangsan Mt., 180619-001[KB]

\section{Symplocaceae 노린재나무과}

378. Symplocos sawafutagi Nagam. 노린재나무 $\mathrm{P}[1,2,3,4]$ Geochang-gun (Sangcheon-ri, Geumwonsan Mt.), 110518156[HNHM]; Hamyang-gun (Sangwon-ri, Gibaeksan Mt.),150605-130 [KB]; Hamyang-gun (Bongjeon-ri, Hwangseoksan Mt.), 150519-003[HNHM]; Geomangsan Mt., 180619-004 [KB]

\section{Oleaceae 물푸레나무과}

^379. Abeliophyllum distichum Nakai 미선나무(식)O[1] Geochang-gun (Sangcheon-ri, Geumwonsan Mt.), 110420001 [HNHM]

380. Chionanthus retusus Lindl. \& Paxton 이팝나무(식)O[3] Hwangseoksan Mt.,180801-035 [KB]

381. Fraxinus chiisanensis Nakai 물들메나무U[2, 4] Hamyang-gun (Sangwon-ri, Gibaeksan Mt.), 151016-082[KB];
Geomangsan Mt., 180418-082 [KB]

382. Fraxinus mandshurica Rupr. 들메나무P, T[2]

Hamyang-gun (Sangwon-ri, Gibaeksan Mt.), 150605-036[KB]

383. Fraxinus rhynchophylla Hance 물푸레나무M, P[1, 2, 3] Geochang-gun (Sangcheon-ri, Geumwonsan Mt.), 110518051 [HNHM]; Geochang-gun (Gohak-ri, Gibaeksan Mt.), 150709-054 [KB]; Hamyang-gun (Hwangsan-ri, Hwangseoksan Mt.), 150423-028[HNHM]

384. Fraxinus sieboldiana Blume 쇠물푸레나무M[1, 2, 3] Geochang-gun (Wolseong-ri, Geumwonsan Mt.), 110514061 [HNHM]; Hamyang-gun (Sangwon-ri, Gibaeksan Mt.), 150605-091 [KB]; Hamyang-gun (Sangwon-ri, Hwangseoksan Mt.), 150423-037[HNHM]

385. Ligustrum obtusifolium Siebold \& Zucc. 쥐똥나무M[1, 2, 3] Geochang-gun (Changseon-ri, Geumwonsan Mt.), 110519146[HNHM]; Hamyang-gun (Sangwon-ri, Gibaeksan Mt.), 150605-219 [KB]; Hamyang-gun (Bongjeon-ri, Hwangseoksan Mt.), 150519-032[HNHM]

\section{Gentianaceae 용담과}

386. Gentiana scabra Bunge용담 $\mathrm{M}, \mathrm{P}[2,3]$

Geochang-gun (Sangcheon-ri, Gibaeksan Mt.), 151015$002[\mathrm{~KB}]$; Hamyang-gun (Bongjeon-ri, Hwangseoksan Mt.), 151022-098 [HNHM]

$\star$ 387. Gentiana triflora Pall. var. japonica(Kusn.) H.Hara 과남풀 $\mathrm{M}[1,2]$

Geochang-gun (Wolseong-ri, Geumwonsan Mt.), 110918066[HNHM]; Hamyang-gun (Sangwon-ri, Gibaeksan Mt.),150917-019 [KB]

388. Gentiana zollingeri Fawc. 큰구슬붕이U[1, 2, 3, 4] Geochang-gun (Sangcheon-ri, Geumwonsan Mt.), 110420029[HNHM]; Hamyang-gun (Sangwon-ri, Gibaeksan Mt.), 150424-009 [KB]; Hamyang-gun (Hwangsan-ri, Hwangseoksan Mt.), 150423-023[HNHM]; Geomangsan Mt., 180418-029 [KB] Apocynaceae 협죽도과

389. Cynanchum paniculatum (Bunge) Kitag. ex H. Hara 산해박 $\mathrm{M}[2,3]$

Geochang-gun (Gohak-ri, Gibaeksan Mt.), 150709-109[KB]; Hamyang-gun (Hwangsan-ri, Hwangseoksan Mt.), 150604-134 [HNHM]

390. Metaplexis japonica (Thunb.) Makino 박주가리E, M, P[3] Hwangseoksan Mt.,180801-077 [KB]

^391. Tylophora floribunda Miq. 왜박주가리U[2] Geochang-gun (Gohak-ri, Gibaeksan Mt.), 150709-023[KB]

Rubiaceae 꼭두서니과

392. Galium bungei Steud. var. trachyspermum(A. Gray) Cufod. 네 잎갈퀴U[2]

Geochang-gun (Gohak-ri, Gibaeksan Mt.), 150709-057[KB]

393. Galium dahuricum Turcz. ex Ledeb. 큰잎갈퀴U[1, 3, 4] Geochang-gun (Sangcheon-ri, Geumwonsan Mt.), 110604096[HNHM]; Hamyang-gun (Bongjeon-ri, Hwangseoksan Mt.),150710-105 [HNHM]; Geomangsan Mt., 180619-205[KB]

$\star$ 394. Galium gracilens (A. Gray) Makino 좀네잎갈퀴U[1, 3]

395. Galium maximowiczii (Kom.) Pobed. 개갈퀴 U[1, 2, 3, 4] Geochang-gun (Sangcheon-ri, Geumwonsan Mt.), 110604022[HNHM]; Hamyang-gun (Sangwon-ri, Gibaeksan Mt.),151016-046 [KB]; Hamyang-gun (Bongjeon-ri, Hwangseoksan Mt.), 150710-042[HNHM]; Geomangsan Mt., 180731-288 [KB]

396. Galium paradoxum Maxim. 두메갈퀴 U [4] Geomangsan Mt.,180619-201 [KB] 
Appendix 1. Continued.

397. Galium pogonanthum Franch. \& Sav. 산갈퀴 U[3, 4] Hamyang-gun (Bongjeon-ri, Hwangseoksan Mt.), 150710039 [HNHM]; Geomangsan Mt., 180619-097 [KB]

398. Galium spurium L.갈퀴덩굴 E, M $[1,3]$ Geochang-gun (Changseon-ri, Geumwonsan Mt.), 110519097[HNHM]; Hamyang-gun (Bongjeon-ri, Hwangseoksan Mt.), 150519-012 [HNHM]

399. Galium verum L.솔나물 P [2]

Geochang-gun (Gohak-ri, Gibaeksan Mt.), 150709-060[KB]

400. Paederia foetida L.계요등 M [2, 3]

Geochang-gun (Gohak-ri, Gibaeksan Mt.), 150812-043[KB];

Hamyang-gun (Sangwon-ri, Hwangseoksan Mt.),150813-015 [HNHM]

401. Rubia argyi (H. Lév. \& Vaniot) H. Hara ex Lauener 꼭두서니 $\mathrm{U}[1,2,3]$

Geochang-gun (Sangcheon-ri, Geumwonsan Mt.), 110830025[HNHM]; Geochang-gun (Gohak-ri, Gibaeksan Mt.), 150812-048 [KB]; Hamyang-gun (Bongjeon-ri, Hwangseoksan Mt.), 151022-091[HNHM]

402. Rubia cordifolia L.갈퀴꼭두서니 M [4] Geomangsan Mt., 180731-031 [KB]

Convolvulaceae 메꽃과

403. Cuscuta australis $\mathrm{R}$. Br. 실새삼 U[3]

Hamyang-gun (Hwangsan-ri, Hwangseoksan Mt.), 151023086[HNHM]

404. Cuscuta japonica Choisy새삼M[2, 3] Geochang-gun (Sangcheon-ri, Gibaeksan Mt.), 151015$045[\mathrm{~KB}]$; Hamyang-gun (Hwangsan-ri, Hwangseoksan Mt.),151023-015 [HNHM]

Boraginaceae 지치과

405. Bothriospermum tenellum (Hornem.) Fisch. \& C. A. Mey. 꽃 받이 $\mathrm{U}[3]$

Hamyang-gun (Bongjeon-ri, Hwangseoksan Mt.), 150519038[HNHM]

406. Trigonotis icumae (Maxim.) Makino 덩굴꽃마리E[1] Geochang-gun (Sangcheon-ri, Geumwonsan Mt.), 110514012 [HNHM]

407. Trigonotis peduncularis ((Trevis.) Benth. ex Baker \& S.Moore 꽃마리 $\mathrm{E}[1,2,3]$

Geochang-gun (Wolseong-ri, Geumwonsan Mt.), 110514036[HNHM]; Hamyang-gun (Sangwon-ri, Gibaeksan Mt.), 150424-028 [KB]; Hamyang-gun (Sangwon-ri, Hwangseoksan Mt.), 150423-073[HNHM]

Verbenaceae 마편초과

408. Callicarpa japonica Thunb. 작살나무 $\mathrm{O}[1,2,3,4]$ Hamyang-gun (Sangwon-ri, Geumwonsan Mt.), 110716083[HNHM]; Geochang-gun (Gohak-ri, Gibaeksan Mt.), 150812-057 [KB]; Hamyang-gun (Bongjeon-ri, Hwangseoksan Mt.), 150710-025[HNHM]; Geomangsan Mt., 180731-136 [KB]

409. Clerodendrum trichotomum Thunb. 누리장나무E, M, P[2, 3, 4] Geochang-gun (Gohak-ri, Gibaeksan Mt.), 150812-131[KB]; Hamyang-gun (Hwangsan-ri, Hwangseoksan Mt.),151023-073 [HNHM]; Geomangsan Mt., 180731-183[KB]

410. Tripora divaricata (Maxim.) P. D. Cantino 누린내풀M[3] Hamyang-gun (Hwangsan-ri, Hwangseoksan Mt.), 151023017[HNHM]

\section{Labiatae 꿀풀과}

411. Agastache rugosa (Fisch. \& Mey.) Kuntze 배초향 E[2, 3] Hamyang-gun (Sangwon-ri, Gibaeksan Mt.), 150917-091[KB];
Hwangseoksan Mt., 160921-178 [KB]

$\star 412$. Ajuga spectabilis Nakai자란초 U [4] Geomangsan Mt., 180619-225 [KB]

413. Clinopodium chinense (Benth.) Kuntze var. parviflorum(Kudô) H.Hara 층층이꽃 $\mathrm{E}[2,3]$

Geochang-gun (Gohak-ri, Gibaeksan Mt.), 150812-128[KB];

Hamyang-gun (Sangwon-ri, Hwangseoksan Mt.),150813-026

[HNHM]

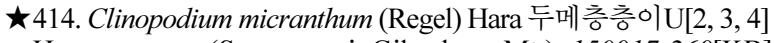
Hamyang-gun (Sangwon-ri, Gibaeksan Mt.), 150917-269[KB]; Hamyang-gun (Bongjeon-ri, Hwangseoksan Mt.), 151022-066 [HNHM]; Geomangsan Mt., 180731-068[KB]

415. Elsholtzia ciliata (Thunb.) Hyl. 향유 M $[1,2]$ Geochang-gun (Wolseong-ri, Geumwonsan Mt.), 110918092[HNHM]; Hamyang-gun (Sangwon-ri, Gibaeksan Mt.),151016-008 [KB]

416. Elsholtzia splendens Nakai ex Maekawa 꽃향유 M[2, 3] Hamyang-gun (Sangwon-ri, Gibaeksan Mt.), 150917-062[KB]; Hamyang-gun (Bongjeon-ri, Hwangseoksan Mt.), 151022-079 [HNHM]

$\star$ 417. Glechoma longituba (Nakai) Kuprian. 긴병꽃풀M[2, 3] Hamyang-gun (Sangwon-ri, Gibaeksan Mt.), 150424-039[KB]; Hamyang-gun (Bongjeon-ri, Hwangseoksan Mt.), 150519-008 [HNHM]

418. Isodon excisus (Maxim.) Kudô 오리방풀E[2, 3] Hamyang-gun (Sangwon-ri, Gibaeksan Mt.), 150918-115[KB]; Hwangseoksan Mt., 160921-010 [KB]

419. Isodon inflexus (Thunb.) Kudô 산박하 E[1, 2, 3, 4] Geochang-gun (Sangcheon-ri, Geumwonsan Mt.), 110830019 [HNHM]; Hamyang-gun (Sangwon-ri, Gibaeksan Mt.), 150917-160 [KB]; Hamyang-gun (Sangwon-ri, Hwangseoksan Mt.), 150813-068[HNHM]; Geomangsan Mt., 180731-090 [KB]

420. Isodon japonicus (Burm.f.) H. Hara 방아풀 $\mathrm{E}[1,3]$ Geochang-gun (Sangcheon-ri, Geumwonsan Mt.), 110830024[HNHM]; Hamyang-gun (Bongjeon-ri, Hwangseoksan Mt.), 151022-003 [HNHM]

421. Lamium album L. subsp. barbatum (Siebold \& Zucc.) Mennema 광대수염E, $\mathrm{P}[1,2,3]$

Hamyang-gun (Sangwon-ri, Geumwonsan Mt.), 110716075[HNHM]; Hamyang-gun (Sangwon-ri, Gibaeksan Mt.), 150424-038 [KB]; Hamyang-gun (Bongjeon-ri, Hwangseoksan Mt.), 150519-009[HNHM]

422. Lamium amplexicaule $\mathrm{L}$. 광대나물 $\mathrm{E}[2,3]$ Hamyang-gun (Sangwon-ri, Gibaeksan Mt.), 150424-067[KB]; Hamyang-gun (Bongjeon-ri, Hwangseoksan Mt.),151022-121 [HNHM]

423. Leonurus japonicus Houtt. 익모초 $\mathrm{M}[2,3]$

Hamyang-gun (Sangwon-ri, Gibaeksan Mt.), 150918-145[KB]; Hamyang-gun (Sangwon-ri, Hwangseoksan Mt.),150813-044 [HNHM]

424. Leonurus macranthus Maxim. 송장풀 M[3] Hwangseoksan Mt., 160921-080 [KB]

Geochang-gun (Sangcheon-ri, Geumwonsan Mt.), 110830-

167[HNHM]; Geochang-gun (Gohak-ri, Gibaeksan Mt.),150812-159

[KB]; Hamyang-gun (Sangwon-ri, Hwangseoksan Mt.), 150813135[HNHM]; Geomangsan Mt., 180731-341 [KB]

425. Lycopus lucidus Turcz. ex Benth. 쉽싸리 M[1, 2, 4] Geochang-gun (Sangcheon-ri, Geumwonsan Mt.), 110830171 [HNHM]; Geochang-gun (Gohak-ri, Gibaeksan Mt.),150709-075 [KB]; Geomangsan Mt., 180731-175[KB] 
Appendix 1. Continued.

426. Meehania urticifolia (Miq.) Makino 벌깨덩굴E, P[1, 4] Geochang-gun (Wolseong-ri, Geumwonsan Mt.), 110514011 [HNHM]; Geomangsan Mt., 180619-052 [KB]

427. Mosla scabra (Thunb.) C. Y. Wu \& H. W. Li 들깨풀 M[1, 3] Geochang-gun (Sangcheon-ri, Geumwonsan Mt.), 110830$292[\mathrm{HNHM}$ ]; Hamyang-gun (Sangwon-ri, Hwangseoksan Mt.),150813-032 [HNHM]

428. Phlomis umbrosa Turcz. 속단E, M[3]

Hamyang-gun (Bongjeon-ri, Hwangseoksan Mt.), 151022063[HNHM]

429. Prunella vulgaris L. subsp. asiatica(Nakai) H. Hara 꿀풀 E, M $[1,2,3,4]$

Geochang-gun (Sangcheon-ri, Geumwonsan Mt.), 110604050[HNHM]; Geochang-gun (Gohak-ri, Gibaeksan Mt.), 150709-186 [KB]; Hamyang-gun (Hwangsan-ri, Hwangseoksan Mt.), 150604-086[HNHM]; Geomangsan Mt., 180731-146 [KB]

430. Salvia plebeia R.Br.배암차즈기 U [2]

Hamyang-gun (Sangwon-ri, Gibaeksan Mt.), 151016-004[KB]

431. Scutellaria indica $\mathrm{L}$. 골무꽃 M [3]

Hwangseoksan Mt., 170525-219 [KB]

432. Scutellaria pekinensis Maxim. var. transitra(Makino) H. Hara 산골무꽃 $\mathrm{U}[1,2,3,4]$

Geochang-gun (Sangcheon-ri, Geumwonsan Mt.), 110604 045[HNHM]; Hamyang-gun (Sangwon-ri, Gibaeksan Mt.), 150605-008 [KB]; Hamyang-gun (Hwangsan-ri, Hwangseoksan Mt.), 150604-014[HNHM]; Geomangsan Mt., 180619-013 [KB]

$\star$ 433. Scutellaria pekinensis Maxim. var. ussuriensis(Regel) Hand.-Mazz. 호골무꽃 U[1]

Geochang-gun (Sangcheon-ri, Geumwonsan Mt.), 110830$246[\mathrm{HNHM}]$

$\star 434$. Teucrium viscidum Blume var. miquelianum(Maxim.) H. Hara 덩굴곽향U $[3,4]$

Hamyang-gun (Sangwon-ri, Hwangseoksan Mt.), 150813 092[HNHM]; Geomangsan Mt., 180731-233 [KB]

Solanaceae 가지과

$\star$ 435. Physaliastrum echinatum (Yatabe) Makino 가시꽈리U[4] Geomangsan Mt., 180731-273 [KB]

436. Solanum lyratum Thunb.배풍등 U [3]

Hamyang-gun (Hwangsan-ri, Hwangseoksan Mt.), 151023 033[HNHM]

437. Solanum nigrum $\mathrm{L}$.까마중 E, M, P [3]

Hamyang-gun (Bongjeon-ri, Hwangseoksan Mt.), 151022 025 [HNHM]

Scrophulariaceae 현삼과

438. Mazus pumilus (Burm.f.) Steenis 주름잎 E[1, 4] Geochang-gun (Changseon-ri, Geumwonsan Mt.), 110519062[HNHM]; Geomangsan Mt., 180418-066 [KB]

439. Melampyrum roseum Maxim. 꽃며느리밥풀 $\mathrm{U}[1,2]$ Geochang-gun (Sangcheon-ri, Geumwonsan Mt.), 110830045[HNHM]; Hamyang-gun (Sangwon-ri, Gibaeksan Mt.), $150917-118$ [KB]

440. Melampyrum roseum Maxim. var. ovalifolium Nakai ex Beauverd 알며느리밥풀U[4] Geomangsan Mt.,180731-088 [KB]

$\star 441$. Mimulus tenellus Bunge var. nepalensis(Benth.) P. C. Tsoong 물꽈리아재비U[3]

Hamyang-gun (Sangwon-ri, Hwangseoksan Mt.), 150813 $101[\mathrm{HNHM}]$

442. Paulownia coreana Uyeki오동나무 (식) T $[1,3]$ Geochang-gun (Wolseong-ri, Geumwonsan Mt.), 110918-
078[HNHM]; Hwangseoksan Mt., 170525-156 [KB]

443. Pedicularis resupinata $\mathrm{L}$. 송이풀 $\mathrm{E}, \mathrm{M}[1,2,4]$ Geochang-gun (Sangcheon-ri, Geumwonsan Mt.), 110830235[HNHM]; Hamyang-gun (Sangwon-ri, Gibaeksan Mt.), 150917-069 [KB]; Geomangsan Mt., 180731-173[KB]

444. Phtheirospermum japonicum (Thunb.) Kanitz 나도송이풀U[2, 3] Hamyang-gun (Sangwon-ri, Gibaeksan Mt.), 150917-215[KB]; Hamyang-gun (Bongjeon-ri, Hwangseoksan Mt.), 151022-101 [HNHM]

445. Pseudolysimachion longifolium(L.) Opiz 긴산꼬리풀U[2 Hamyang-gun (Sangwon-ri, Gibaeksan Mt.), 150917-017[KB]

446. Scrophularia buergeriana Miq. 현삼 M [3]

Hamyang-gun (Sangwon-ri, Hwangseoksan Mt.), 150813067[HNHM]

447. Veronica polita $\mathrm{Fr}$.개불알풀 U [4] Geomangsan Mt., 180418-072 [KB]

\section{Phrymaceae 파리풀과}

448. Phryma leptostachya L. var. oblongifolia(Koidz.) Honda 파리 풀 $\mathrm{M}[1,2,3,4]$

Hamyang-gun (Sangwon-ri, Geumwonsan Mt.), 110716-

127[HNHM]; Geochang-gun (Gohak-ri, Gibaeksan Mt.), 150812-201 [KB]; Hamyang-gun (Bongjeon-ri,

Hwangseoksan Mt.), 150710-103[HNHM]; Geomangsan Mt., 180731-013 [KB]

\section{Plantaginaceae 질경이과}

449. Plantago asiatica L. 질경이 E, M, P [1, 2, 3, 4] Geochang-gun (Sangcheon-ri, Geumwonsan Mt.), 110604025 [HNHM]; Hamyang-gun (Sangwon-ri, Gibaeksan Mt.), 150605-058 [KB]; Hamyang-gun (Bongjeon-ri, Hwangseoksan Mt.), 150710-083[HNHM]; Geomangsan Mt., 180619-238 [KB]

$\star 450$. Plantago depressa Willd. 털질경 이E, M, P[1] Geochang-gun (Sangcheon-ri, Geumwonsan Mt.), 110519 $025[\mathrm{HNHM}]$

Caprifoliaceae 인동과

451. Lonicera japonica Thunb. 인동덩굴 $\mathrm{M}, \mathrm{P}[2,3]$ Geochang-gun (Gohak-ri, Gibaeksan Mt.), 150709-174[KB]; Hamyang-gun (Hwangsan-ri, Hwangseoksan Mt.),150604-067 [HNHM]

452. Sambucus racemosa L. subsp. kamtschatica(E. Wolf) Hultén 지렁쿠나무 $\mathrm{E}, \mathrm{M}, \mathrm{P}[1,2,3]$

Geochang-gun (Wolseong-ri, Geumwonsan Mt.), 110514054[HNHM]; Hamyang-gun (Sangwon-ri, Gibaeksan Mt.), 150605-014 [KB]; Hamyang-gun (Hwangsan-ri, Hwangseoksan Mt.), 150423-020[HNHM]

453. Viburnum opulus var. calvescens (Rehder) H. Hara 백당나무 $\mathrm{O}[1,2]$

Geochang-gun (Sangcheon-ri, Geumwonsan Mt.), 110604 097[HNHM]; Hamyang-gun (Sangwon-ri, Gibaeksan Mt.), 150605-127 [KB]

454. Weigela florida (Bunge) A. DC. 붉은병꽃나무O, $\mathrm{P}[2]$ Hamyang-gun (Sangwon-ri, Gibaeksan Mt.), 150917-212[KB]

455. Weigela subsessilis (Nakai) L.H.Bailey 병꽃나무O, $\mathrm{P}[1,2,3,4]$ Geochang-gun (Wolseong-ri, Geumwonsan Mt.), 110514080 [HNHM]; Hamyang-gun (Sangwon-ri, Gibaeksan Mt.), 150424-085 [KB]; Hamyang-gun (Hwangsan-ri, Hwangseoksan Mt.), 150423-009[HNHM]; Geomangsan Mt., 180619-107 [KB]

Valerianaceae 마타리과

456. Patrinia saniculaefolia Hemsl. 금마타리U[2] Hamyang-gun (Sangwon-ri, Gibaeksan Mt.), 150917-250[KB] 
Appendix 1. Continued.

457. Patrinia scabiosaefolia Fisch. ex Trevir. 마타리 E, M, P $[1,2,3,4]$

458. Patrinia villosa (Thunb.) Juss. 뚝갈 $\mathrm{E}[1,2,3,4]$

Geochang-gun (Sangcheon-ri, Geumwonsan Mt.), 110830-

063[HNHM]; Geochang-gun (Gohak-ri, Gibaeksan

Mt.),150812-088 [KB]; Hamyang-gun (Bongjeon-ri,

Hwangseoksan Mt.), 151022-104[HNHM]; Geomangsan Mt., 180731-006 [KB]

459. Valeriana fauriei Briq.쥐오줌풀 E [1]

Geochang-gun (Wolseong-ri, Geumwonsan Mt.), 110514001 [HNHM]

\section{Campanulaceae 초롱꽃과}

460. Adenophora remotiflora (Siebold \& Zucc.) Miq. 모시대 E, $\mathrm{M}[1,2]$

Geochang-gun (Sangcheon-ri, Geumwonsan Mt.), 110830232[HNHM]; Hamyang-gun (Sangwon-ri, Gibaeksan Mt.), 150918-093 [KB]

461. Adenophora triphylla (Thunb.) A. DC. var. japonica(Regel) H. Hara 잔대 E, M [2, 3, 4]

Geochang-gun (Gohak-ri, Gibaeksan Mt.), 150709-204[KB]; Hwangseoksan Mt., 160922-044 [KB]; Geomangsan Mt., 180731-264 [KB]

462. Adenophora verticillata Fisch. var. hirsutaF. Schmidt 털잔대 $\mathrm{E}[3]$

Hamyang-gun (Sangwon-ri, Hwangseoksan Mt.), 150813114 [HNHM]

463. Asyneuma japonicum (Miq.) Briq. 영아자 E[1, 2, 3, 4] Geochang-gun (Sangcheon-ri, Geumwonsan Mt.), 110830211 [HNHM]; Geochang-gun (Gohak-ri, Gibaeksan Mt.), 150812110 [KB]; Hamyang-gun (Sangwon-ri, Hwangseoksan Mt.), 150813-118[HNHM]; Geomangsan Mt., 180731-102 [KB]

464. Codonopsis lanceolata (Siebold \& Zucc.) Benth. \& Hook. f. ex Trautv. 더덕 (식) E, M, P [3]

Hwangseoksan Mt., 170525-111 [KB]

$\star 465$. Codonopsis ussuriensis (Rupr. \& Maxim.) Hemsl. 소경불알E[3] Hamyang-gun (Sangwon-ri, Hwangseoksan Mt.), 150813076[HNHM]

$\star$ 466. Lobelia chinensis Lour.수염 가래꽃 M [2] Geochang-gun (Gohak-ri, Gibaeksan Mt.), 150812-173[KB]

467. Platycodon grandiflorum (Jacq.) A. DC. 도라지 (식)E, M, $\mathrm{O}[3]$

Hwangseoksan Mt., 180801-097 [KB]

\section{Compositae 국화과}

468. Adenocaulon himalaicum Edgew. 멸가치 E[3] Hwangseoksan Mt., 160922-067 [KB]

469. Ainsliaea acerifolia Sch. Bip. 단풍취 $\mathrm{E}[1,2,3]$ Geochang-gun (Sangcheon-ri, Geumwonsan Mt.), 110830209 [HNHM]; Hamyang-gun (Sangwon-ri, Gibaeksan Mt.), 150917-051 [KB]; Hwangseoksan Mt., 160922019 [HNHM]

470. Ambrosia artemisiifolia L. 돼지풀 U[3]

Hamyang-gun (Bongjeon-ri, Hwangseoksan Mt.), 151022134 [HNHM]

471. Artemisia capillaris Thunb. 사철쑥 $\mathrm{E}, \mathrm{P}[2,3]$ Geochang-gun (Sangcheon-ri, Gibaeksan Mt.), 151015075[KB]; Hamyang-gun (Sangwon-ri, Hwangseoksan Mt.),150813-027 [HNHM]

472. Artemisia japonica Thunb. 제비쑥 $\mathrm{E}, \mathrm{P}[2]$

Hamyang-gun (Sangwon-ri, Gibaeksan Mt.), 150917-133[KB]

473. Artemisia keiskeana Miq. 맑은대쑥E, M, P[1, 2, 3] Geochang-gun (Sangcheon-ri, Geumwonsan Mt.), 110830-
291 [HNHM]; Hamyang-gun (Sangwon-ri, Gibaeksan

Mt.), 150917-188 [KB]; Hamyang-gun (Bongjeon-ri,

Hwangseoksan Mt.), 151022-075[HNHM]

474. Artemisia montana (Nakai) Pamp. 산쑥 P $[2,3]$

Hamyang-gun (Sangwon-ri, Gibaeksan Mt.), 150917-134[KB];

Hamyang-gun (Bongjeon-ri, Hwangseoksan Mt.),151022-031 [HNHM]

475. Artemisia stolonifera (Maxim.) Kom. 넓은잎외잎쑥E, $\mathrm{P}[1,2,3]$ Geochang-gun (Sangcheon-ri, Geumwonsan Mt.), 110830202[HNHM]; Hamyang-gun (Sangwon-ri, Gibaeksan Mt.), 150917-049 [KB]; Hamyang-gun (Bongjeon-ri, Hwangseoksan Mt.), 151022-022[HNHM]

476. Aster ageratoides Turcz. 까실쑥부쟁이 U[1, 2, 3] Geochang-gun (Wolseong-ri, Geumwonsan Mt.), 110918063[HNHM]; Geochang-gun (Gohak-ri, Gibaeksan Mt.), 150709-132 [KB]; Hamyang-gun (Bongjeon-ri, Hwangseoksan Mt.), 151022-051[HNHM]

$\star 477$. Aster incisus Fisch.가새쑥부쟁이 U $[1,2]$ Geochang-gun (Sangcheon-ri, Geumwonsan Mt.), 110830289 [HNHM]; Geochang-gun (Gohak-ri, Gibaeksan Mt.), $150812-176$ [KB]

478. Aster koraiensis Nakai벌개미취 E [1] Geochang-gun (Sangcheon-ri, Geumwonsan Mt.), 110830068[HNHM]

479. Aster meyendorfii (Regel \& Maack) Voss 개쑥부쟁이U[1, 2, 3] Geochang-gun (Sangcheon-ri, Geumwonsan Mt.), 110830078[HNHM]; Hamyang-gun (Sangwon-ri, Gibaeksan Mt.), 150917-123 [KB]; Hamyang-gun (Hwangsan-ri, Hwangseoksan Mt.), 150604-005[HNHM]

480. Aster scaber Thunb.참취E, $\mathrm{P}[1,2,3,4]$

Geochang-gun (Sangcheon-ri, Geumwonsan Mt.), 110830018[HNHM]; Geochang-gun (Gohak-ri, Gibaeksan Mt.), 150812-107 [KB]; Hamyang-gun (Bongjeon-ri, Hwangseoksan Mt.), 151022-095[HNHM]; Geomangsan Mt., 180731-126 [KB]

481. Atractylodes ovata (Thunb.) DC. 삽주 E, M, P [2, 3] Geochang-gun (Sangcheon-ri, Gibaeksan Mt.), 151015 $003[\mathrm{~KB}]$; Hamyang-gun (Hwangsan-ri, Hwangseoksan Mt.), 151023-048 [HNHM]

482. Bidens bipinnata $\mathrm{L}$.도깨비바늘 $\mathrm{E}, \mathrm{M}, \mathrm{P}$ [3]

Hamyang-gun (Bongjeon-ri, Hwangseoksan Mt.), 151022088[HNHM]

483. Bidens tripartita L.가막사리 E, M [2, 3]

Hamyang-gun (Sangwon-ri, Gibaeksan Mt.), 151016-059[KB]; Hamyang-gun (Bongjeon-ri, Hwangseoksan Mt.),151022-089 [HNHM]

484. Carpesium abrotanoides L. 담배풀 M[2] Hamyang-gun (Sangwon-ri, Gibaeksan Mt.), 150918-069[KB]

485. Carpesium divaricatum Siebold \& Zucc. 긴담배풀E, M[2, 4] Geochang-gun (Gohak-ri, Gibaeksan Mt.), 150812-009[KB]; Geomangsan Mt., 180731-257 [KB]

^486. Carpesium glossophyllum Maxim. 천일담배풀U[3] Hamyang-gun (Bongjeon-ri, Hwangseoksan Mt.), 151022117 [HNHM]

487. Carpesium triste Maxim. 두메담배풀 U [3, 4] Hwangseoksan Mt., 160921-020 [KB]; Geomangsan Mt., $180731-323[\mathrm{~KB}]$

488. Chrysanthemum boreale (Makino) Makino 산국 M, P [2, 3] Geochang-gun (Sangcheon-ri, Gibaeksan Mt.), 151015066[KB]; Hamyang-gun (Bongjeon-ri, Hwangseoksan Mt.), 151022-083 [HNHM] 
Appendix 1. Continued.

489. Chrysanthemum zawadskii Herbich 산구절초M, P[1] Geochang-gun (Wolseong-ri, Geumwonsan Mt.), 110918048 [HNHM]

490. hrysanthemum zawadskii Herbich var. latilobum(Maxim.) Kitam. 구절초 $\mathrm{M}, \mathrm{P}[1,2,3]$

Geochang-gun (Sangcheon-ri, Geumwonsan Mt.), 110830286[HNHM]; Hamyang-gun (Sangwon-ri, Gibaeksan Mt.), 150917-058 [KB]; Hamyang-gun (Bongjeon-ri, Hwangseoksan Mt.), 151022-094[HNHM]

491. Cirsium chanroenicum (L.) Nakai 정 영엉 겅퀴U[1] Geochang-gun (Wolseong-ri, Geumwonsan Mt.), 110918059 [HNHM]

492. Cirsium japonicum Fisch. ex DC. var. maackii(Maxim.) Matsum. 엉겅퀴 E, M, P[1, 2, 3, 4] Geochang-gun (Changseon-ri, Geumwonsan Mt.), 110519171 [HNHM]; Geochang-gun (Gohak-ri, Gibaeksan Mt.), 150709-187 [KB]; Hamyang-gun (Hwangsan-ri, Hwangseoksan Mt.), 150604-085[HNHM]; Geomangsan Mt., 180731-001 [KB]

^493. Cirsium pendulum Fisch. ex DC. 큰엉겅 퀴E[2] Hamyang-gun (Sangwon-ri, Gibaeksan Mt.), 150917-267[KB]

494. Cirsium setidens (Dunn) Nakai 고려엉겅 퀴E[1, 2, 3] Geochang-gun (Sangcheon-ri, Geumwonsan Mt.), 110830287[HNHM]; Hamyang-gun (Sangwon-ri, Gibaeksan Mt.), 150917-126 [KB]; Hwangseoksan Mt., 160921-082[KB]

495. Conyza canadensis (L.) Cronquist 망초 E [3]

Hwangseoksan Mt., 180801-038 [KB]

496. Cosmos bipinnatus Cav.코스모스 U [3]

Hwangseoksan Mt., 160922-011 [KB]

497. Crepidiastrum chelidoniifolium(Makino) J. H. Pak \& Kawano 까치고들빼기 $\mathrm{E}[1,2,3]$

Geochang-gun (Sangcheon-ri, Geumwonsan Mt.), 110830111 [HNHM]; Hamyang-gun (Sangwon-ri, Gibaeksan Mt.), 150917-025 [KB]; Hwangseoksan Mt., 160921-124[KB]

498. Crepidiastrum denticulatum(Houtt.) J. H. Pak \& Kawano이고 들빼기 $\mathrm{E}, \mathrm{P}[1,2,3]$

Geochang-gun (Wolseong-ri, Geumwonsan Mt.), 110918001 [HNHM]; Hamyang-gun (Sangwon-ri, Gibaeksan Mt.), 150917-163 [KB]; Hamyang-gun (Hwangsan-ri, Hwangseoksan Mt.), 151023-066[HNHM]

499. Crepidiastrum koidzumianum(Kitam.) J.H.Pak \& Kawano지 리고들빼기 U [2]

Hamyang-gun (Sangwon-ri, Gibaeksan Mt.), 150917-149[KB]

500. Crepidiastrum sonchifolium(Maxim.) J. H. Pak \& Kawano고 들빼기 $\mathrm{E}, \mathrm{P}[1,3]$

Geochang-gun (Sangcheon-ri, Geumwonsan Mt.), 110518005 [HNHM]; Hamyang-gun (Bongjeon-ri, Hwangseoksan Mt.),150519-010 [HNHM]

501. Erechtites hieraciifolius (L.) Raf. ex DC. 붉은서나물E[3] Hamyang-gun (Hwangsan-ri, Hwangseoksan Mt.), 151023085 [HNHM]

502. Erigeron annuus (L.) Pers. 개 망초 E[1, 2, 3, 4] Geochang-gun (Sangcheon-ri, Geumwonsan Mt.), 110604 049 [HNHM]; Hamyang-gun (Sangwon-ri, Gibaeksan Mt.), 150605-184 [KB]; Hamyang-gun (Hwangsan-ri, Hwangseoksan Mt.), 150604-095[HNHM]; Geomangsan Mt., 180619-127 [KB]

503. Euchiton japonicus (Thunb.) Holub 풀솜나물 $\mathrm{E}[4]$ Geomangsan Mt.,180731-122 [KB]

504. Eupatorium japonicum Thunb. 등골나물E, $\mathrm{P}[1,2,3,4]$ Hamyang-gun (Sangwon-ri, Geumwonsan Mt.), 110716-
061 [HNHM]; Geochang-gun (Gohak-ri, Gibaeksan Mt.), 150709-124 [KB]; Hamyang-gun (Sangwon-ri, Hwangseoksan Mt.), 150813-113[HNHM]; Geomangsan Mt., 180731-140 [KB]

505. Eupatorium lindleyanum DC. 골등골나물E, $\mathrm{P}[3]$

Hamyang-gun (Hwangsan-ri, Hwangseoksan Mt.), 151023078[HNHM]

$\star$ 506. Eupatorium makinoi Kawah. \& Yahara var. oppositifolium(Koidz.) Kawah. \& Yahara 벌등골나물 E, P [3, 4] Hamyang-gun (Hwangsan-ri, Hwangseoksan Mt.), 151023 036[HNHM]; Geomangsan Mt., 180731-178 [KB]

507. Galinsoga ciliata (Raf.) S.F.Blake 털별 꽃아재비U[3, 4] Hamyang-gun (Sangwon-ri, Hwangseoksan Mt.), 150813052[HNHM]; Geomangsan Mt., 180731-196 [KB]

$\star$ 508. Galinsoga parviflora Cav. 별꽃아재비U[2] Geochang-gun (Gohak-ri, Gibaeksan Mt.), 150709-198[KB]

509. Hemistepta lyrata (Bunge) Bunge 지칭개 $\mathrm{E}, \mathrm{P}[1,2,3]$ Geochang-gun (Sangcheon-ri, Geumwonsan Mt.), 110830248 [HNHM]; Hamyang-gun (Sangwon-ri, Gibaeksan Mt.), 150605-054 [KB]; Hamyang-gun (Bongjeon-ri, Hwangseoksan Mt.), 150519-029[HNHM]

510. Hieracium umbellatum L.조밥나물 E, P [2, 3] Hamyang-gun (Sangwon-ri, Gibaeksan Mt.), 150917-056[KB]; Hamyang-gun (Sangwon-ri, Hwangseoksan Mt.),150813-034 [HNHM]

511. Ixeridium dentatum (Thunb.) Tzvelev 씀바귀 E, M, P[1,3] Geochang-gun (Sangcheon-ri, Geumwonsan Mt.), 110604047[HNHM]; Hamyang-gun (Hwangsan-ri, Hwangseoksan Mt.),150604-128 [HNHM]

$\star$ 512. Ixeris chinensis (Thunb.) Nakai 노랑선씀바귀 U[3] Hamyang-gun (Hwangsan-ri, Hwangseoksan Mt.), 150604 058[HNHM]

513. Ixeris polycephala Cass. 벌씀바귀 E, $\mathrm{P}[1,2,3]$ Geochang-gun (Changseon-ri, Geumwonsan Mt.), 110519 042[HNHM]; Hamyang-gun (Sangwon-ri, Gibaeksan Mt.), 151016-011 [KB]; Hwangseoksan Mt., 170525-237[KB]

514. Ixeris strigosa (H.Lev. \& Vaniot) J. H. Pak \& Kawano 선씀바 귀E, $\mathrm{P}[1,2,3]$ Geochang-gun (Sangcheon-ri, Geumwonsan Mt.), 110604 057[HNHM]; Geochang-gun (Gohak-ri, Gibaeksan Mt.), 150709-108 [KB]; Hwangseoksan Mt., 180801-149[KB]

515. Lactuca indica L.왕고들빼기 E, M, P [1, 2, 3] Geochang-gun (Wolseong-ri, Geumwonsan Mt.), 110918088[HNHM]; Hamyang-gun (Sangwon-ri, Gibaeksan Mt.),150917-046 [KB]; Hamyang-gun (Sangwon-ri, Hwangseoksan Mt.), 150813-035[HNHM]

516. Leibnitzia anandria (L.) Turcz. 솜나물 E, P[2] Hamyang-gun (Sangwon-ri, Gibaeksan Mt.), 150917-077[KB]

517. Petasites japonicus (Siebold \& Zucc.) Maxim. 머위 E, M, P [3] Hamyang-gun (Sangwon-ri, Hwangseoksan Mt.), 150423085[HNHM]

518. Pseudognaphalium affine (D. Don) Anderb. 떡쑥 E, M [3] Hamyang-gun (Hwangsan-ri, Hwangseoksan Mt.), 150604144[HNHM]

519. Rudbeckia bicolor Nutt.원추천인국 O [4] Geomangsan Mt.,180731-010 [KB]

$\star 520$. Rudbeckia laciniata L.삼잎국화 O [4] Geomangsan Mt.,180731-344 [KB]

521. Saussurea gracilis Maxim. 은분취 $\mathrm{P}[2]$ Hamyang-gun (Sangwon-ri, Gibaeksan Mt.), 150917-016[KB] 
Appendix 1. Continued.

522. Serratula coronata L. subsp. insularis(Iljin) Kitam. 산비장이 $\mathrm{E}[1,2]$

Geochang-gun (Sangcheon-ri, Geumwonsan Mt.), 110830-

123[HNHM]; Hamyang-gun (Sangwon-ri, Gibaeksan Mt.), 150917-073 [KB]

523. Sigesbeckia glabrescens (Makino) Makino 진득찰 M, P[2] Hamyang-gun (Sangwon-ri, Gibaeksan Mt.), 150918-192[KB]

524. Solidago virgaurea L. subsp. asiaticaKitam. ex H. Hara 미역 취 $\mathrm{E}, \mathrm{M}[1,2,3]$

Geochang-gun (Sangcheon-ri, Geumwonsan Mt.), 110830136[HNHM]; Hamyang-gun (Sangwon-ri, Gibaeksan Mt.), 150917-057 [KB]; Hamyang-gun (Sangwon-ri, Hwangseoksan Mt.), 150813-061[HNHM]

Geochang-gun (Changseon-ri, Geumwonsan Mt.), 110519053 [HNHM]

525. Syneilesis palmata (Thunb.) Maxim. 우산나물 $\mathrm{U}[1,3,4]$ Geochang-gun (Sangcheon-ri, Geumwonsan Mt.), 110830129 [HNHM]; Hwangseoksan Mt., 160921-081 [KB]; Geomangsan Mt., 180731-306[KB]

526. Synurus deltoides (Aiton) Nakai 수리취 E, P $[1,2]$ Geochang-gun (Wolseong-ri, Geumwonsan Mt.), 110918060[HNHM]; Hamyang-gun (Sangwon-ri, Gibaeksan Mt.), 150917-003 [KB]

$\star 527$. Synurus excelsus (Makino) Kitam. 큰수리취E, P[1] Geochang-gun (Wolseong-ri, Geumwonsan Mt.), 110918$073[\mathrm{HNHM}]$

528. Taraxacum officinale Weber 서양민들레 $\mathrm{E}[1,2,3]$ Geochang-gun (Wolseong-ri, Geumwonsan Mt.), 110514026[HNHM]; Hamyang-gun (Sangwon-ri, Gibaeksan Mt.), 150424-054 [KB]; Hamyang-gun (Sangwon-ri, Hwangseoksan Mt.), 150423-084[HNHM]

$\star$ 529. Taraxacum ohwianum Kitam. 산민들레 $\mathrm{E}[2,3]$ Hamyang-gun (Sangwon-ri, Gibaeksan Mt.), 150424-059[KB]; Hamyang-gun (Hwangsan-ri, Hwangseoksan Mt.),151023-084 [HNHM]

530. Taraxacum platycarpum Dahlst. 민들레 E, M[1] Geochang-gun (Changseon-ri, Geumwonsan Mt.), 110519$110[\mathrm{HNHM}]$

531. Tephroseris kirilowii (Turcz. ex DC.) Holub 솜방망이E[1] Geochang-gun (Sangcheon-ri, Geumwonsan Mt.), 110519002 [HNHM]

532. Tephroseris pseudosonchus (Vaniot) C. Jeffrey \& Y. L. Chen 물솜방망이U[1]

Geochang-gun (Sangcheon-ri, Geumwonsan Mt.), 110518 094[HNHM]

533. Youngia japonica (L.) DC. 뽀리뱅이 E[1, 2, 3] Geochang-gun (Sangcheon-ri, Geumwonsan Mt.), 110518146[HNHM]; Hamyang-gun (Sangwon-ri, Gibaeksan Mt.), 150424-031 [KB]; Hamyang-gun (Sangwon-ri, Hwangseoksan Mt.), 150423-049[HNHM]

Alismataceae 택사과

$\star$ 534. Sagittaria aginashi Makino 보풀 U [2] Geochang-gun (Gohak-ri, Gibaeksan Mt.), 150812-020[KB] Liliaceae 백합과

535. Allium macrostemon Bunge 산달래 E, M[1]

Geochang-gun (Changseon-ri, Geumwonsan Mt.), 110519 004 [HNHM]

536. Allium monanthum Maxim. 달래E[4]

Geomangsan Mt.,180419-021 [KB]

537. Allium thunbergii G. Don 산부추 $\mathrm{E}[2,3]$
Hamyang-gun (Sangwon-ri, Gibaeksan Mt.), 150917-064[KB]; Hamyang-gun (Bongjeon-ri, Hwangseoksan Mt.),150519-026 [HNHM]

538. Asparagus schoberioides Kunth 비짜루 E[3]

Hamyang-gun (Hwangsan-ri, Hwangseoksan Mt.), 150604119 [HNHM]

539. Barnardia japonica (Thunb.) Schult. f. 무릇 E [2, 3$]$ Geochang-gun (Gohak-ri, Gibaeksan Mt.), 150812-179[KB]; Hamyang-gun (Sangwon-ri, Hwangseoksan Mt.),150813-038 [HNHM]

540. Convallaria keiskei Miq. 은방울꽃 M[2, 3] Geochang-gun (Gohak-ri, Gibaeksan Mt.), 150709-103[KB]; Hamyang-gun (Hwangsan-ri, Hwangseoksan Mt.), 150604-117 [HNHM]

541. Disporum smilacinum A. Gray 애기나리E, $\mathrm{P}[1,2,3]$ Geochang-gun (Wolseong-ri, Geumwonsan Mt.), 110514007[HNHM]; Hamyang-gun (Sangwon-ri, Gibaeksan Mt.), 150917-176 [KB]; Hwangseoksan Mt., 170525-245[HNHM]

542. Disporum uniflorum Baker 윤판나물E, $\mathrm{P}[1,2,3]$ Geochang-gun (Sangcheon-ri, Geumwonsan Mt.), 110518176[HNHM]; Hamyang-gun (Sangwon-ri, Gibaeksan Mt.), 150918-014 [KB]; Hamyang-gun (Hwangsan-ri, Hwangseoksan Mt.), 150604-044[HNHM]

543. Disporum viridescens (Maxim.) Nakai 큰애기나리E, $\mathrm{P}[1,3,4]$ Geochang-gun (Sangcheon-ri, Geumwonsan Mt.), 110515052[HNHM]; Hamyang-gun (Bongjeon-ri, Hwangseoksan Mt.),150710-100 [HNHM]; Geomangsan Mt., 180619-179[KB]

544. Heloniopsis koreana Fuse, N. S. Lee \& M. N. Tamura 처녀치마U[1] Geochang-gun (Sangcheon-ri, Geumwonsan Mt.), 110420025 [HNHM]

545. Hemerocallis fulva (L.) L. 원추리 E, M, O, P[1] Geochang-gun (Sangcheon-ri, Geumwonsan Mt.), 110830077[HNHM]

$\star$ 546. Hemerocallis hakuunensis Nakai 백 운산원추리E, M, O, $\mathrm{P}[1,2,3,4]$

Geochang-gun (Sangcheon-ri, Geumwonsan Mt.), 110830173[HNHM]; Hamyang-gun (Sangwon-ri, Gibaeksan Mt.),150917-041 [KB]; Hamyang-gun (Bongjeon-ri, Hwangseoksan Mt.), 151022-073[HNHM]; Geomangsan Mt., 180731-085 [KB]

547. Hosta capitata (Koidz.) Nakai 일 월비비추E, P[1, 2, 3, 4] Geochang-gun (Sangcheon-ri, Geumwonsan Mt.), 110604059 [HNHM]; Geochang-gun (Gohak-ri, Gibaeksan Mt.),150709-131 [KB]; Hamyang-gun (Bongjeon-ri, Hwangseoksan Mt.), 150710-004[HNHM]; Geomangsan Mt., 180731-243 [KB]

548. Lilium amabile Palib. 털중나리 E, M [1, 2, 3] Hamyang-gun (Sangwon-ri, Geumwonsan Mt.), 110716031 [HNHM]; Hamyang-gun (Sangwon-ri, Gibaeksan Mt.),150917-081 [KB]; Hamyang-gun (Hwangsan-ri, Hwangseoksan Mt.), 150604-037[HNHM]

549. Lilium lancifolium Thunb. 참나리 E, $\mathrm{M}[1,2]$ Hamyang-gun (Sangwon-ri, Geumwonsan Mt.), 110716089[HNHM]; Geochang-gun (Gohak-ri, Gibaeksan Mt.), 150709-190 [KB]

550. Lilium tsingtauense Gilg 하늘말나리E, M, O, P[1, 2, 3] Geochang-gun (Sangcheon-ri, Geumwonsan Mt.), 110604072[HNHM]; Geochang-gun (Gohak-ri, Gibaeksan Mt.),150812-012 [KB]; Hamyang-gun (Bongjeon-ri, Hwangseoksan Mt.), 150710-014[HNHM] 
Appendix 1. Continued.

551. Liriope spicata (Thunb.) Lour. 개맥문동M, $\mathrm{P}[2,3,4]$ Geochang-gun (Gohak-ri, Gibaeksan Mt.), 150812-181[KB]; Hamyang-gun (Bongjeon-ri, Hwangseoksan Mt.),151022-108 [HNHM]; Geomangsan Mt., 180731-119[KB]

552. Lloydia triflora (Ledeb.) Baker 나도개감채U[4] Geomangsan Mt.,180419-015 [KB]

553. Maianthemum japonicum (A. Gray) LaFrankie 풀솜대 E[1, 4] Geochang-gun (Sangcheon-ri, Geumwonsan Mt.), 110420011 [HNHM]; Geomangsan Mt., 180619-165 [KB]

554. Paris verticillata $\mathrm{M}$. Bieb. 삿갓나물 $\mathrm{E}[1,3,4]$ Geochang-gun (Sangcheon-ri, Geumwonsan Mt.), 110514028[HNHM]; Hamyang-gun (Sangwon-ri, Hwangseoksan Mt.),150423-024 [HNHM]; Geomangsan Mt., 180619-142[KB]

$\star$ 555. Polygonatum humile Fisch. ex Maxim. 각시둥굴레E, P[1] Geochang-gun (Wolseong-ri, Geumwonsan Mt.), 110514013 [HNHM]

556. Polygonatum inflatum Kom. 퉁둥굴레 U[1,3] Geochang-gun (Changseon-ri, Geumwonsan Mt.), 110519001 [HNHM]; Hamyang-gun (Hwangsan-ri, Hwangseoksan Mt.),150604-041 [HNHM]

557. Polygonatum involucratum (Franch. \& Sav.) Maxim. 용둥굴 레E, P[1]

558. Polygonatum lasianthum Maxim. 죽대 $\mathrm{M}[1,3]$ Geochang-gun (Sangcheon-ri, Geumwonsan Mt.), 110515004[HNHM]; Hamyang-gun (Bongjeon-ri, Hwangseoksan Mt.),151022-001 [HNHM]

559. Polygonatum odoratum var. pluriflorum(Miq.) Ohwi 둥굴레 E, $\mathrm{P}[1,2,3,4]$

Geochang-gun (Wolseong-ri, Geumwonsan Mt.), 110514018[HNHM]; Geochang-gun (Gohak-ri, Gibaeksan Mt.), 150709-188 [KB]; Hamyang-gun (Hwangsan-ri, Hwangseoksan Mt.), 150604-002[HNHM]; Geomangsan Mt., 180619-168 [KB]

560. Smilax china L.청미래덩굴 E, M, P [1, 2, 3]

Geochang-gun (Sangcheon-ri, Geumwonsan Mt.), 110518199 [HNHM]; Hamyang-gun (Sangwon-ri, Gibaeksan Mt.), 150424-005 [KB]; Hamyang-gun (Hwangsan-ri, Hwangseoksan Mt.), 150423-026[HNHM]

561. Smilax nipponica Miq.선밀나물 E, P [1,3] Geochang-gun (Sangcheon-ri, Geumwonsan Mt.), 110518068 [HNHM]; Hamyang-gun (Hwangsan-ri, Hwangseoksan Mt.),150423-032 [HNHM]

562. Smilax riparia A. DC. 밀나물 E, P [3]

Hamyang-gun (Hwangsan-ri, Hwangseoksan Mt.), 150604 113 [HNHM]

$\star$ 563. Smilax sieboldii Miq. f. inermis(Nakai ex T. Mori) H.Hara 민청가시덩굴 $\mathrm{E}, \mathrm{P}[2,3]$

Geochang-gun (Gohak-ri, Gibaeksan Mt.), 150709-146[KB];

Hamyang-gun (Hwangsan-ri, Hwangseoksan Mt.),151023-034 [HNHM]

564. Tricyrtis macropoda Miq. 뻐꾹나리E[1, 2, 3] Geochang-gun (Sangcheon-ri, Geumwonsan Mt.), 110830087[HNHM]; Geochang-gun (Gohak-ri, Gibaeksan Mt.), $150812-059$ [KB]; Hamyang-gun (Hwangsan-ri, Hwangseoksan Mt.), 150604-197[HNHM]

565. Veratrum maackii Regel var. japonicum(Baker) Schmizu 여로 $\mathrm{M}$ [1]

Hamyang-gun (Sangwon-ri, Geumwonsan Mt.), 110716032[HNHM]

$\star$ 566. Veratrum maackii Regel var. parviflorum(Maxim.) H. Hara 파란여로 $\mathrm{M}[2,3]$
Geochang-gun (Gohak-ri, Gibaeksan Mt.), 150812-092[KB]; Hamyang-gun (Bongjeon-ri, Hwangseoksan Mt.),150710-122 [HNHM]

567. Veratrum versicolor Nakai 흰여로 U[1]

Hamyang-gun (Sangwon-ri, Geumwonsan Mt.), 120721 $002[\mathrm{KH}]$

\section{Dioscoreaceae 마과}

568. Dioscorea japonica Thunb. 참마 E, M [2]

Geochang-gun (Gohak-ri, Gibaeksan Mt.), 150709-127[KB]

569. Dioscorea nipponica Makino 부채마 E, P[1]

Hamyang-gun (Sangwon-ri, Geumwonsan Mt.), 110716008 [HNHM]

570. Dioscorea tenuipes Franch. \& Sav. 각시마 M[2, 3, 4] Geochang-gun (Gohak-ri, Gibaeksan Mt.), 150709-009[KB]; Hwangseoksan Mt., 180801-084, Geomangsan Mt.,180731-189 [HNHM]

\section{Pontederiaceae 물옥잠과}

571. Monochoria vaginalis (Burm.f.) C.Presl var. plantaginea(Roxb.) Solms 물달개비U[2]

Geochang-gun (Gohak-ri, Gibaeksan Mt.), 150812-183[KB]

\section{Iridaceae 붓꽃과}

572. Iris ensata Thunb. var. spontanea(Makino) Nakai 꽃창포 O, $\mathrm{P}[1,2]$

Geochang-gun (Sangcheon-ri, Geumwonsan Mt.), 110518-

140 [HNHM]; Hamyang-gun (Sangwon-ri, Gibaeksan

Mt.), 150605-159 [KB]

$\star 573$. Iris koreana Nakai노랑붓꽃 (식) O, P [3]

Hwangseoksan Mt., 170525-182 [KB]

574. Iris rossii Baker각시붓꽃 O, P [3]

Hamyang-gun (Hwangsan-ri, Hwangseoksan Mt.), 150423 019 [HNHM]

575. Iris sanguinea Donn ex Hornem. 붓꽃 $\mathrm{M}, \mathrm{O}, \mathrm{P}[1,2,3]$ Geochang-gun (Sangcheon-ri, Geumwonsan Mt.), 110604063[HNHM]; Geochang-gun (Gohak-ri, Gibaeksan

Mt.), 150709-193 [KB]; Hamyang-gun (Hwangsan-ri,

Hwangseoksan Mt.), 150604-137[HNHM]

\section{Juncaceae 골풀과}

576. Juncus decipiens (Buchenau) Nakai 골풀 M, P [2, 3, 4] Geochang-gun (Gohak-ri, Gibaeksan Mt.), 150709-069[KB];

Hamyang-gun (Hwangsan-ri, Hwangseoksan Mt.),150604-165

[HNHM]; Geomangsan Mt., 180731-295[KB]

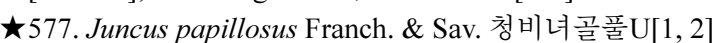

Geochang-gun (Sangcheon-ri, Geumwonsan Mt.), 110830-

165[HNHM]; Geochang-gun (Gohak-ri, Gibaeksan

Mt.),150812-146 [KB]

$\star 578$. Juncus tenuis Willd.길골풀 U [3]

Hamyang-gun (Hwangsan-ri, Hwangseoksan Mt.), 150604$159[\mathrm{HNHM}]$

579. Luzula capitata (Miq. ex Franch. \& Sav.) Kom. 꿩의밥 U[1, 2, 3, 4] Geochang-gun (Sangcheon-ri, Geumwonsan Mt.), 110515005[HNHM]; Hamyang-gun (Sangwon-ri, Gibaeksan Mt.), 150424-010 [KB]; Hamyang-gun (Sangwon-ri, Hwangseoksan Mt.), 150423-082[HNHM]; Geomangsan Mt., 180418-049 [KB]

Commelinaceae 닭의장풀과

580. Commelina communis L.닭의장풀 E, M, P [1, 2, 3] Geochang-gun (Sangcheon-ri, Geumwonsan Mt.), 110830091 [HNHM]; Geochang-gun (Gohak-ri, Gibaeksan Mt.), 150709-012 [KB]; Hamyang-gun (Hwangsan-ri, Hwangseoksan Mt.), 150604-136[HNHM] 
Appendix 1. Continued.

$\star$ 581. Commelina communis L. var. angustifolia Nakai 좀닭의장 풀E, $\mathrm{M}, \mathrm{P}[1]$

Hamyang-gun (Sangwon-ri, Geumwonsan Mt.), 110716115 [HNHM]

582. Streptolirion volubile Edgew. 덩굴닭의장풀E[2]

Geochang-gun (Gohak-ri, Gibaeksan Mt.), 150812-084[KB]

Gramineae 벼과

583. Achnatherum pekinense (Hance) Ohwi 나래새 P [3, 4]

Hamyang-gun (Hwangsan-ri, Hwangseoksan Mt.), 151023 012[HNHM]; Geomangsan Mt., 180731-302 [KB]

584. Alopecurus aequalis Sobol. 뚝새풀 $\mathrm{P}[1]$

Geochang-gun (Changseon-ri, Geumwonsan Mt.), 110519046[HNHM]

585. Arthraxon hispidus (Thunb.) Makino 조개풀 $\mathrm{P}$ [3] Hamyang-gun (Bongjeon-ri, Hwangseoksan Mt.), 151022 084 [HNHM]

586. Arundinella hirta (Thunb.) Tanaka var. ciliata(Thunb.) Koidz. 새 P $[2,3,4]$

Hamyang-gun (Sangwon-ri, Gibaeksan Mt.), 150917-117[KB]; Hwangseoksan Mt., 160921-027 [KB]; Geomangsan Mt., 180731-026 [KB]

^587. Brachypodium sylvaticum (Huds.) P.Beauv. 숲개밀 P[4] Geomangsan Mt., 180619-159 [KB]

$\star 588$. Bromus catharticus Vahl큰이삭풀 U [3] Hwangseoksan Mt., 170525-176 [HNHM]

589. Bromus remotiflorus (Steud.) Ohwi 꼬리새 P[1] Hamyang-gun (Sangwon-ri, Geumwonsan Mt.), 110716$062[\mathrm{HNHM}]$

590. Calamagrostis arundinacea (L.) Roth 실새풀 $\mathrm{P}[1,2,3]$ Geochang-gun (Sangcheon-ri, Geumwonsan Mt.), 110830004[HNHM]; Hamyang-gun (Sangwon-ri, Gibaeksan Mt.),150918-112 [KB]; Hamyang-gun (Bongjeon-ri, Hwangseoksan Mt.), 151022-017[HNHM]

591. Dactylis glomerata L.오리새 P [2, 3] Hamyang-gun (Sangwon-ri, Gibaeksan Mt.), 150605-077[KB]; Hwangseoksan Mt., 170526-106 [HNHM]

592. Diarrhena japonica (Franch. \& Sav.) Franch. \& Sav. 용수염 $\mathrm{P}[1,3]$

Hamyang-gun (Sangwon-ri, Geumwonsan Mt.), 120721035[KH]; Hamyang-gun (Bongjeon-ri, Hwangseoksan Mt.),151022-002 [HNHM]

$\star$ 593. Diarrhena mandshurica Maxim. 껍질용수염 P[1] Hamyang-gun (Sangwon-ri, Geumwonsan Mt.), 110716093[HNHM]

594. Digitaria ciliaris (Retz.) Koeler 바랭이 P[3] Hamyang-gun (Bongjeon-ri, Hwangseoksan Mt.), 151022086[HNHM]

595. Echinochloa crus-galli (L.) P. Beauv. 돌피 E, S [3] Hamyang-gun (Bongjeon-ri, Hwangseoksan Mt.), 151022036 [HNHM]

596. Agropyron ciliare (Trin.) Franch. 속털개밀 P[3] Hwangseoksan Mt.,170525-105 [KB]

597. Eragrostis ferruginea (Thunb.) P. Beauv. 그령 P [1, 3] Geochang-gun (Wolseong-ri, Geumwonsan Mt.), 110918$010[\mathrm{HNHM}]$; Hamyang-gun (Bongjeon-ri, Hwangseoksan Mt.),151022-138 [HNHM]

598. Eragrostis multicaulis Steud. 비노리 P[4] Geomangsan Mt.,180731-018 [KB]

599. Festuca arundinacea Schreb. 큰김의털U[1] Geochang-gun (Sangcheon-ri, Geumwonsan Mt.), 110604-
001 [HNHM]

$\star 600$. Festuca rubra L.왕김의털 P [3]

Hamyang-gun (Hwangsan-ri, Hwangseoksan Mt.), 150604096[HNHM]

601. Lolium perenne L.호밀풀 U [3]

Hamyang-gun (Sangwon-ri, Hwangseoksan Mt.), 150813104 [HNHM]

$\star$ 602. Microstegium vimineum (Trin.) A. Camus 나도바랭이새P[1] Geochang-gun (Wolseong-ri, Geumwonsan Mt.), 110918093[HNHM]

603. Milium effusum L.나도겨이삭 P [3, 4]

Hamyang-gun (Bongjeon-ri, Hwangseoksan Mt.), 150710 -

127[HNHM]; Geomangsan Mt., 180619-251 [KB]

604. Miscanthus sinensis Andersson var. purpurascens(Andersson) Matsum. 억새 P [2, 3]

Hamyang-gun (Sangwon-ri, Gibaeksan Mt.), 150917-258[KB]; Hamyang-gun (Bongjeon-ri, Hwangseoksan Mt.),151022-137 [HNHM]

605. Muhlenbergia japonica Steud. 쥐꼬리새P[2]

Hamyang-gun (Sangwon-ri, Gibaeksan Mt.), 150917-042[KB]

$\star 606$. Oplismenus burmanni (Retz.) P. Beauv. 민주름조개풀P[1] Geochang-gun (Sangcheon-ri, Geumwonsan Mt.), 110830278 [HNHM]

607. Oplismenus undulatifolius (Ard.) P. Beauv. 주름조개풀P $[1,2,4]$ Geochang-gun (Sangcheon-ri, Geumwonsan Mt.), 110830-258[HNHM]; Geochang-gun (Gohak-ri, Gibaeksan Mt.),150812-148 [KB]; Geomangsan Mt., 180731-336[KB]

608. Panicum bisulcatum Thunb. 개기장 E, P[3] Hwangseoksan Mt.,160922-060 [KB]

609. Paspalum thunbergii Kunth ex Steud. 참새피 M, P[3, 4] Hamyang-gun (Bongjeon-ri, Hwangseoksan Mt.), 151022141 [HNHM]; Geomangsan Mt., 180731-116 [KB]

610. Pennisetum alopecuroides (L.) Spreng. 수크령 P[2, 3] Hamyang-gun (Sangwon-ri, Gibaeksan Mt.), 150918-146[KB]; Hwangseoksan Mt., 160922-055 [KB]

611.Poa acroleuca Steud.실포아풀 P [1]

Geochang-gun (Changseon-ri, Geumwonsan Mt.), 110519028 [HNHM]

612. Poa annua L.새포아풀 P [3]

Hamyang-gun (Bongjeon-ri, Hwangseoksan Mt.), 151022045 [HNHM]

613. Poa pratensis L.왕포아풀 P [3] Hwangseoksan Mt.,180619-185 [KIOM]

614. Sasa borealis (Hack.) Makino \& Shibata 조릿대 E, M, P[1, 2, 3, 4] Geochang-gun (Sangcheon-ri, Geumwonsan Mt.), 110518$060[\mathrm{HNHM}]$; Hamyang-gun (Sangwon-ri, Gibaeksan Mt.), 150424-076 [KB]; Hamyang-gun (Sangwon-ri, Hwangseoksan Mt.), 150423-006[HNHM]; Geomangsan Mt., 180619-241 [KB]

615. Setaria viridis (L.) P. Beauv. 강아지풀P[3, 4] Hwangseoksan Mt.,180801-119 [KB]; Geomangsan Mt., 180731-052[KB]

616. Spodipogon cotulifer (Thunb.) Hack. 기름새 P[2] Hamyang-gun (Sangwon-ri, Gibaeksan Mt.), 150917-109[KB]

617. Spodipogon sibiricus Trin. 큰기름새 $\mathrm{P}[3,4]$ Hwangseoksan Mt.,160921-092 [KB]; Geomangsan Mt., 180731-083[KB]

618. Trisetum bifidum (Thunb.) Ohwi 잠자리피 $\mathrm{P}[1,3]$ Geochang-gun (Changseon-ri, Geumwonsan Mt.), 110519152[HNHM]; Hwangseoksan Mt., 170525-099 [KB] 
Appendix 1. Continued.

\begin{tabular}{l}
\hline \hline Araceae 천남성과 \\
^619. Arisaema amurense Maxim. 둥근잎천남성 M, P[1, 2, 3, 4] \\
Geochang-gun (Changseon-ri, Geumwonsan Mt.), 110519- \\
111 [HNHM]; Hamyang-gun (Sangwon-ri, Gibaeksan \\
Mt.),150424-018 [KB]; Hamyang-gun (Sangwon-ri, \\
Hwangseoksan Mt.), 150423-035[HNHM]; Geomangsan Mt., \\
180418-074 [KB] \\
620. Arisaema amurense Maxim. f. serratum(Nakai) Kitag. 천남성 \\
$\mathrm{M}, \mathrm{P}[1,3]$ \\
Geochang-gun (Changseon-ri, Geumwonsan Mt.), 110519- \\
$114[\mathrm{HNHM}$; Hamyang-gun (Hwangsan-ri, Hwangseoksan Mt.), \\
150604-034 [HNHM]
\end{tabular}

621. Arisaema serratum (Thunb.) Schott 점박이천남성 M, $\mathrm{P}[1,2,3,4]$ Geochang-gun (Changseon-ri, Geumwonsan Mt.), 110519010 [HNHM]; Geochang-gun (Gohak-ri, Gibaeksan Mt.), 150812-034 [KB]; Hamyang-gun (Sangwon-ri, Hwangseoksan Mt.), 150813-057[HNHM]; Geomangsan Mt., 180731-215 [KB]

622. Pinellia ternata (Thunb.) Makino 반하 M $[1,2]$

Geochang-gun (Changseon-ri, Geumwonsan Mt.), 110519099[HNHM]; Hamyang-gun (Sangwon-ri, Gibaeksan Mt.), 150605-081 [KB]

Cyperaceae 사초과

$\star 623$. Carex aphanolepis Franch. \& Sav. 골사초 P[3, 4] Hwangseoksan Mt.,180619-178 [KIOM], Geomangsan Mt., 180619-094[KB]

$\star 624$. Carex biwensis Franch. \& Sav. 솔잎사초P[1] Geochang-gun (Changseon-ri, Geumwonsan Mt.), 110519065 [HNHM]

Hamyang-gun (Sangwon-ri, Hwangseoksan Mt.), 150423071 [HNHM]

625. Carex bostrychostigma Maxim. 길뚝사초P[3] Hwangseoksan Mt., 170526-024 [KB]

626. Carex breviculmis $\mathrm{R}$. $\mathrm{Br}$. 청사초 $\mathrm{P}[1,3,4]$

Geochang-gun (Changseon-ri, Geumwonsan Mt.), 110519109 [HNHM]; Hamyang-gun (Sangwon-ri, Hwangseoksan Mt.),150423-041 [HNHM]; Geomangsan Mt., 180418-114[KB]

$\star 627$. Carex capillacea Boott잔솔잎사초 P [1] Geochang-gun (Sangcheon-ri, Geumwonsan Mt.), 110830251 [HNHM]

$\star$ 628. Carex doniana Spreng.흰사초 P [4] Geomangsan Mt., 180731-284 [KB]

$\star 629$. Carex fernaldiana H. Lev. \& Vaniot 실사초 P[3] Hwangseoksan Mt., 170525-028 [KB]

$\star 630$. Carex forficula Franch. \& Sav. 산뚝사초P[1] Geochang-gun (Sangcheon-ri, Geumwonsan Mt.), 110518138 [HNHM]

631. Carex gibba Wahlenb.나도별사초 P [3]

Hwangseoksan Mt., 170525-101 [KB]

632. Carex japonica Thunb.개찌버리사초 $\mathrm{P}[1,3]$

Geochang-gun (Sangcheon-ri, Geumwonsan Mt.), 110519$009[$ HNHM]; Hamyang-gun (Hwangsan-ri, Hwangseoksan Mt.), 150604-098 [HNHM]

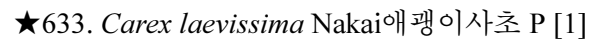
Geochang-gun (Sangcheon-ri, Geumwonsan Mt.), 110518197[HNHM]

1, Geumwonsan Mt.; 2, Gibaeksan Mt.; 3, Hwangseoksan Mt.; 4, Geomangsan Mt. E, edible plant; F, fiber plant; M, medicinal plant; O, ornamental plant; P, pasture plant; R, industrial raw material plant; S, stain plant; T, timber source plant; U, unknown use plant; The number behind scientific name means voucher specimen number submitted to National History Museum, Hannam University[HNHM], Korea National Arboretum[KH], National Institute of Biological Resources[KB], Korean Institute of Oriental Medicine[KIOM]; ?, Newly found taxa in this investigation.
634. Carex lanceolata Boott그늘사초 P $[2,3,4]$

Hamyang-gun (Sangwon-ri, Gibaeksan Mt.), 150424-024[KB];

Hamyang-gun (Sangwon-ri, Hwangseoksan Mt.),150423-070

[HNHM]; Geomangsan Mt., 180418-013[KB]

635. Carex leiorhyncha C.A.Mey. 산갱이사초 $\mathrm{P}[1,2,3,4]$ Geochang-gun (Sangcheon-ri, Geumwonsan Mt.), 110604027[HNHM]; Hamyang-gun (Sangwon-ri, Gibaeksan Mt.), 150605-061 [KB]; Hamyang-gun (Hwangsan-ri, Hwangseoksan Mt.), 150604-139[HNHM]; Geomangsan Mt., 180619-043 [KB]

636. Carex maximowiczii Miq.왕비늘사초 $\mathrm{P}[1,3]$

Geochang-gun (Changseon-ri, Geumwonsan Mt.), 110519-

154[HNHM]; Hwangseoksan Mt., 170525-108 [KB]

$\star$ 637. Carex papulosa Boott쇠낚시사초 P [3]

$\star$ 638. Carex polyschoena H. Lév. \& Vaniot 가지청사초P[1] 008 [HNHM]

$\star$ 639. Carex sabynensis Less. ex Kunth 실청사초P[1, 3] Geochang-gun (Wolseong-ri, Geumwonsan Mt.), 110514004[HNHM]; Hwangseoksan Mt., 170526-108 [KB]

640. Carex siderosticta Hance 대사초 P $[1,3,4]$

Geochang-gun (Sangcheon-ri, Geumwonsan Mt.), 110515017[HNHM]; Hamyang-gun (Sangwon-ri, Hwangseoksan Mt.),150423-031 [HNHM]; Geomangsan Mt., 180419-061[KB]

641. Cyperus amuricus Maxim. 방동사니 M [2]

Geochang-gun (Gohak-ri, Gibaeksan Mt.), 150709-039[KB]

642. Cyperus iria L.참방동사니 U $[2,3]$

Hwangseoksan Mt., 180801-095 [KB]

643. Scirpus karuizawensis Makino 솔방울고랭이U[1]

Geochang-gun (Sangcheon-ri, Geumwonsan Mt.), 110830269[HNHM]

644. Scirpus wichurae Boeck. 방울고랭이 U [3] 028 [HNHM]

Orchidaceae 난초과

645. Cephalanthera erecta (Thunb.) Blume 은난초 U[1] Geochang-gun (Sangcheon-ri, Geumwonsan Mt.), 110519034[HNHM]

646. Cephalanthera longibracteataBlume 은대난초U[1, 2, 3, 4] Geochang-gun (Sangcheon-ri, Geumwonsan Mt.), 110518081 [HNHM]; Geochang-gun (Gohak-ri, Gibaeksan Mt.),150709-102 [KB]; Hamyang-gun (Hwangsan-ri, Hwangseoksan Mt.), 150604-061[HNHM]; Geomangsan Mt., 180731-109 [KB]

647. Gastrodia elata Blume천마M[3] Hwangseoksan Mt.,170714-041 [KB]

648. Liparis kumokiri F. Maek. 옥잠난초U[1, 2, 3] Geochang-gun (Sangcheon-ri, Geumwonsan Mt.), 110830138[HNHM]; Hamyang-gun (Sangwon-ri, Gibaeksan Mt.),150917-047 [KB]; Hamyang-gun (Hwangsan-ri, Hwangseoksan Mt.), 150604-203[HNHM]

$\star 649$. Oreorchis patens (Lindl.) Lindl. 감자난초U[1, 4] Geochang-gun (Sangcheon-ri, Geumwonsan Mt.), 110514008[HNHM]; Geomangsan Mt., 180619-215 [KB] Geochang-gun (Sangcheon-ri, Geumwonsan Mt.), 110519-

Hamyang-gun (Sangwon-ri, Gibaeksan Mt.), 150917-031[KB];

Hamyang-gun (Sangwon-ri, Hwangseoksan Mt.), 150813- 